\title{
LOOMIS-WHITNEY-TYPE INEQUALITIES AND LOW REGULARITY WELL-POSEDNESS OF THE PERIODIC ZAKHAROV-KUZNETSOV EQUATION
}

\author{
SHINYA KINOSHITA AND ROBERT SCHIPPA
}

\begin{abstract}
Local well-posedness for the two-dimensional Zakharov-Kuznetsov equation in the fully periodic case with initial data in Sobolev spaces $H^{s}$, $s>1$, is proved. Frequency dependent time localization is utilized to control the derivative nonlinearity. The new ingredient to improve on previous results is a nonlinear Loomis-Whitney-type inequality.
\end{abstract}

\section{INTRODUCTION}

The purpose of this article is to improve local well-posedness of the ZakharovKuznetsov equation with periodic boundary conditions in two dimensions

$$
\left\{\begin{array}{cl}
\partial_{t} u+\left(\partial_{x_{1}}^{3}+\partial_{x_{1}} \partial_{x_{2}}^{2}\right) u & =u \partial_{x_{1}} u, \quad(t, x) \in \mathbb{R} \times \mathbb{T}^{2}, \\
u(0) & =u_{0} \in H_{\mathcal{R}}^{s}\left(\mathbb{T}^{2}\right),
\end{array}\right.
$$

where $\mathbb{T}=\mathbb{R} /(2 \pi \mathbb{Z})$, and $H_{\mathcal{R}}^{s}$ denotes the Sobolev space with regularity index $s$ comprised of real-valued functions.

By local well-posedness we mean that the data-to-solution mapping $S_{T}^{\infty}: H_{\mathcal{R}}^{\infty} \rightarrow$ $C\left([0, T], H_{\mathcal{R}}^{\infty}\right)$ for $T=T\left(\left\|u_{0}\right\|_{H_{\mathcal{R}}^{s}}\right)$ assigning smooth, real-valued initial data to smooth, real-valued solutions admits an extension to a continuous mapping $S_{T}^{s}$ : $H_{\mathcal{R}}^{s} \rightarrow C\left([0, T], H_{\mathcal{R}}^{s}\right)$.

The Zakharov-Kuznetsov equation in three dimensions was derived in [19] to describe unidirectional ionic-sonic wave propagation in a magnetized plasma. Laedke and Spatschek derived also the two-dimensional model from the equations of motions for hydrodynamics in [20], which was further justified in [21] by LannesLinares-Saut.

As a higher-dimensional analog of the Korteweg-de Vries equation

$$
\partial_{t} u+\partial_{x}^{3} u=u \partial_{x} u
$$

(1) has also been extensively studied, and the body of literature is huge. In the following we aim to deliver an overview of the well-posedness theory for (1) in two dimensions.

Conserved quantities for real-valued solutions are the mass

$$
M(u)=\int u_{0}^{2} d x
$$

and energy

$$
E(u)=\int \frac{|\nabla u|^{2}}{2}+\frac{u^{3}}{3} d x .
$$

2010 Mathematics Subject Classification. 35Q53, 42 B37.

Key words and phrases. Zakharov-Kuznetsov equation, local well-posedness, short-time Fourier restriction norm method, nonlinear Loomis-Whitney inequality. 
In Euclidean space the Zakharov-Kuznetsov equation is invariant under the scaling

$$
u(t, x) \rightarrow \lambda^{2} u\left(\lambda^{3} t, \lambda x\right),
$$

which distinguishes $s_{c}=-1$ as scaling critical regularity.

The classical energy method (cf. [8]) gives local well-posedness in $H_{\mathcal{R}}^{s}, s>2$ as well on $\mathbb{R}^{2}$ as $\mathbb{T}^{2}$. In Euclidean space this was subsequently improved making use of dispersive effects. In [12] global well-posedness was proved in $H_{\mathcal{R}}^{1}\left(\mathbb{R}^{2}\right)$. In this work smoothing and maximal function estimates were used to solve the ZakharovKuznetsov equation via the contraction mapping principle (cp. [16] for the earlier application in context of the Korteweg-de Vries equation). Linares and Pastor improved local well-posedness to $s>3 / 4$ in [23] by refining the proof in [12]. In the works [26] and [13] due to Molinet-Pilod and Grünrock-Herr, bilinear Strichartz estimates were used to prove local well-posedness for $s>1 / 2$.

Recently, the first author proved local well-posedness in $H^{s}\left(\mathbb{R}^{2}\right)$ for $s>-1 / 4$ in [17]. The improvement stems from the use of the nonlinear Loomis-Whitney inequality to derive refined multilinear estimates for fully transverse interactions. The result from [17] is sharp up to endpoints in the sense that the data-to-solution mapping fails to be $C^{2}$ for $s<-1 / 4$. The literature for Loomis-Whitney inequalities is vast (see e.g. [24, 9, 7, 3, 5, 2, 18]); however, for many results on abstract Loomis-Whitney inequalities the application to nonlinear dispersive equations is not clear, as transversality or size of the involved hypersurfaces is not quantified precisely. The nonlinear Loomis-Whitney inequality in $\mathbb{R}^{3}$ with scalable assumptions on the hypersurfaces was investigated in [3]; see also [1] for an application to the Zakharov system and $[2,5]$ for subsequent higher-dimensional progress. A strengthened form of the nonlinear Loomis-Whitney inequality is given by multilinear restriction inequalities; see [6] and the references therein. In [6], the dependence on the transversality was not quantified. This was only recently accomplished in three dimensions in [27].

Due to decreased dispersion, the periodic case is worse behaved: in the work [22] by Linares et al. was shown that (1) is not amenable to Picard iteration for $s>1 / 2$, provided that (1) is locally well-posed at all for $s>1 / 2$. In fact, local well-posedness was proved for $s>5 / 3$ by short-time linear Strichartz estimates in [22].

This was modestly improved by the second author to $s>3 / 2$ via short-time bilinear Strichartz estimates adapting the bilinear arguments from $[13,26]$ to the periodic case in [28]. Thus, the natural question is to what extend the refined approach from [17] leads to improved local well-posedness on $\mathbb{T}^{2}$. We prove the following theorem:

Theorem 1.1. Let $s>1$. Then, we find (1) to be locally well-posed.

Remark 1.2. Our result is sensitive with respect to the periods. The proof does not extend to the torus $\sqrt{3} \lambda \mathbb{T} \times \lambda \mathbb{T}$ with $\lambda>0$, but to all ratinal tori. We refer to Section 5 for further details. In Section 5 we shall also see that $s=1$ is the limit of our method of frequency dependent time localization and transversality considerations.

Furthermore, the local well-posedness results on $\mathbb{R}^{2}$ proved via the contraction mapping principle hold for complex initial data. In Section 6 we prove norm inflation for complex initial data on $\mathbb{T}^{2}$. In the following we consider local well-posedness for (1) implicitly only for real-valued initial data. 
Short-time analysis was used in the periodic case in [22] and [28] as it was pointed out in [22] that (1) is not amenable to Picard iteration. The function spaces used in the present work were introduced for the Euclidean space in [15]. The construction in the periodic case will be revisited in Section 2. By now there are many works related with frequency dependent time localization. We refer to the expositions in $[22,28,29]$ and the references therein for a more complete depiction. To deal with large initial data, we rescale the torus to handle small initial data on large tori. Thus, we will also consider estimates on tori with arbitrary periods. In the context of short-time analysis this was previously done in [25]; see also Section 2.

For the proof of Theorem 1.1 we will show the following sets of estimates. Let $\lambda \geq 1$ denote the period length and $1<s \leq s^{\prime}$ the regularity and $T \in(0,1]$. Firstly, for smooth solutions $u \in C\left([0, T], H_{\lambda}^{s}\right)$ emanating from $\lambda$-periodic smooth initial data $u_{0} \in H_{\lambda}^{\infty}$ we find the following estimates to hold:

$$
\left\{\begin{aligned}
\|u\|_{F_{\lambda}^{s^{\prime}}(T)} & \lesssim\|u\|_{E_{\lambda}^{s^{\prime}}(T)}+\left\|u \partial_{x_{1}} u\right\|_{N_{\lambda}^{s^{\prime}}(T)} \\
\left\|u \partial_{x_{1}} u\right\|_{N_{\lambda}^{s^{\prime}}(T)} & \lesssim\|u\|_{F_{\lambda}^{s^{\prime}}(T)}\|u\|_{F_{\lambda}^{s}(T)} \\
\|u\|_{E_{\lambda}^{s^{\prime}}(T)}^{2} & \lesssim\left\|u_{0}\right\|_{H_{\lambda}^{s^{\prime}}}^{2}+\|u\|_{F_{\lambda}^{s}(T)}\|u\|_{F_{\lambda}^{s^{\prime}}(T)}^{2}
\end{aligned}\right.
$$

By standard bootstrap arguments this proves a priori estimates and persistence of regularity on $[0, T]$ for small initial data in $H_{\lambda}^{s}$.

For differences of solutions $v=u_{1}-u_{2}$, with smooth initial data $u_{i}(0) \in H_{\lambda}^{\infty}$ and $1<s$, we show

$$
\left\{\begin{array}{cl}
\|v\|_{F_{\lambda}^{0}(T)} & \lesssim\|v\|_{E_{\lambda}^{0}(T)}+\left\|\partial_{x_{1}}\left(v\left(u_{1}+u_{2}\right)\right)\right\|_{N_{\lambda}^{0}(T)}, \\
\left\|\partial_{x_{1}}\left(v\left(u_{1}+u_{2}\right)\right)\right\|_{N_{\lambda}^{0}(T)} & \lesssim\|v\|_{F_{\lambda}^{0}(T)}\left\|u_{1}+u_{2}\right\|_{F_{\lambda}^{s}(T)}, \\
\|v\|_{E_{\lambda}^{0}(T)}^{2} & \lesssim\|v(0)\|_{L_{\lambda}^{2}}^{2}+\|v\|_{F_{\lambda}^{0}(T)}^{2}\left(\left\|u_{1}\right\|_{F_{\lambda}^{s}(T)}+\left\|u_{2}\right\|_{F_{\lambda}^{s}(T)}\right) .
\end{array}\right.
$$

This proves Lipschitz-continuous dependence in $L_{\lambda}^{2}$ for small initial data in $H_{\lambda}^{s}$.

By virtue of the following set of estimates,

$$
\left\{\begin{array}{cl}
\|v\|_{F_{\lambda}^{s^{\prime}}(T)} & \lesssim\|v\|_{E_{\lambda}^{s^{\prime}}(T)}+\left\|\partial_{x_{1}}\left(v\left(u_{1}+u_{2}\right)\right)\right\|_{N_{\lambda}^{s^{\prime}}(T)} \\
\left\|\partial_{x_{1}}\left(v\left(u_{1}+u_{2}\right)\right)\right\|_{N_{\lambda}^{s^{\prime}}(T)} & \lesssim\|v\|_{F_{\lambda}^{s^{\prime}}(T)}\left(\left\|u_{1}\right\|_{F_{\lambda}^{s}(T)}+\left\|u_{2}\right\|_{F_{\lambda}^{s}(T)}\right) \\
\|v\|_{E_{\lambda}^{s^{\prime}}(T)}^{2} & \lesssim\|v(0)\|_{H_{\lambda}^{s^{\prime}}}^{2}+\left(\|v\|_{F_{\lambda}^{0}(T)}\|v\|_{F_{\lambda}^{s^{\prime}}(T)}\left\|u_{2}\right\|_{F_{\lambda}^{2 s^{\prime}}(T)}\right. \\
& \left.+\|v\|_{F_{\lambda}^{s^{\prime}}(T)}^{2}\left\|u_{2}\right\|_{F_{\lambda}^{s}(T)}+\|v\|_{F_{\lambda}^{s^{\prime}}(T)}^{2}\|v\|_{F_{\lambda}^{s}(T)}\right)
\end{array}\right.
$$

continuous dependence for small initial data in $H_{\lambda}^{s}$ follows via the classical BonaSmith approximation (cf. [8]). The reduction from arbitrary initial data in $H^{s}\left(\mathbb{T}^{2}\right)$ to initial data with small Sobolev norm on $\lambda \mathbb{T}^{2}$ is carried out via scaling. For previous applications of scaling in the context of frequency dependent time localization applied to periodic solutions; see e.g. [25, 29].

The linear estimate, propagating $u, v$, respectively, in $F_{\lambda}^{s}(T)$ is recalled in Section 2. The short-time nonlinear estimate propagating the nonlinearity in $N_{\lambda}^{s}(T)$ was carried out in [28] and is recalled in Section 3. The first part of Section 4 is devoted to the global nonlinear Loomis-Whitney inequality on $\mathbb{R}^{3}$. After that Loomis-Whitneytype inequalities on $\mathbb{R} \times$ lattices which play a crucial role in the proof of energy estimates are discussed. For the energy estimate in Section 5, the analysis from [28] is refined with the aid of the transversality considerations from [17]. In Section 6 , we prove norm inflation for periodic complex initial data with arbitrary Sobolev regularity, which is not the case in $\mathbb{R}^{2}$. 
With the above sets of estimates at disposal, the proof of Theorem 1.1 is concluded by standard bootstrap arguments, which are omitted. For details, we refer to $[28]$.

\section{Notation}

Dyadic numbers will be denoted by capital letters $N \in 2^{\mathbb{N}_{0}}$, where $\mathbb{N}_{0}=\mathbb{N} \cup\{0\}$. For $\xi \in \mathbb{R}^{n}$ let $|\xi|=\sqrt{\xi_{1}^{2}+\ldots+\xi_{n}^{2}}$ denote the Euclidean norm and $\langle\xi\rangle^{2}=1+|\xi|^{2}$. Set $\mathbb{T}=\mathbb{R} /(2 \pi \mathbb{Z})$ and for $\lambda \geq 1$ set $\lambda \mathbb{T}^{n}=\lambda \mathbb{T} \times \ldots \times \lambda \mathbb{T}$ and $\mathbb{Z}^{n} / \lambda=\mathbb{Z} / \lambda \times \ldots \times \mathbb{Z} / \lambda$. Varying $\lambda$ we have to be aware of possible dependencies of constants on the spatial scale. Let $(d \xi)_{\lambda}$ be the normalized counting measure on $\mathbb{Z}^{n} / \lambda$ :

$$
\int a(\xi)(d \xi)_{\lambda}:=\lambda^{-n} \sum_{\xi \in \mathbb{Z}^{n} / \lambda} a(\xi) .
$$

The Fourier transform on $\lambda \mathbb{T}^{n}$ is defined for $f \in L^{1}\left(\lambda \mathbb{T}^{n} ; \mathbb{C}\right)$ by

$$
\hat{f}(k)=\int_{\lambda \mathbb{T}^{n}} e^{-i k \cdot x} f(x) d x, \quad k \in \mathbb{Z}^{n} / \lambda .
$$

The inverse Fourier transform is given by

$$
\check{g}(x)=\frac{1}{(2 \pi)^{n}} \int g(\xi) e^{i x \cdot \xi}(d \xi)_{\lambda} .
$$

The usual properties like Plancherel's theorem or Parseval's identity of the Fourier transform hold. We refer to [11, p. 727] for further properties.

Let $\chi: \mathbb{R} \rightarrow \mathbb{R}_{\geq 0}$ denote a smooth symmetric function, supported in $[-7 / 8,7 / 8]$ with $\chi \equiv 1$ on $[-5 / 4,5 / 4]$ and set $\chi_{k}(\xi)=\chi\left(2^{-k}|\xi|\right)-\chi\left(2^{1-k}|\xi|\right)$ for $k \in \mathbb{N}$. Note that

$$
\sum_{k=1}^{\infty} \chi_{k}(\xi)+\chi(\xi) \equiv 1
$$

For $N=2^{n}, n \in \mathbb{N}_{0}$ we denote by $P_{N}$ the Littlewood-Paley projector associated with $\chi_{n}$, i.e.,

$$
\left(P_{N} f\right) \widehat{(\xi)}=\chi_{n}(|\xi|) \hat{f}(\xi)
$$

. We define Sobolev spaces for $s \geq 0$ as

$$
H^{s}\left(\lambda \mathbb{T}^{n}\right)=\left\{\left.f \in L^{2}\left(\lambda \mathbb{T}^{n}\right)\left|\|f\|_{H_{\lambda}^{s}}^{2}=\int\langle\xi\rangle^{2 s}\right| \hat{f}(\xi)\right|^{2}(d \xi)_{\lambda}<\infty\right\}
$$

and $H^{\infty}\left(\lambda \mathbb{T}^{n}\right)=\bigcap_{s \geq 0} H^{s}\left(\lambda \mathbb{T}^{n}\right)$.

We turn to the definition of the short-time $X^{s, b}$-spaces. Let $\eta_{0}: \mathbb{R} \rightarrow[0,1]$ denote an even, smooth function with $\eta_{0} \equiv 1$ on $[-5 / 4,5 / 4]$ on $\operatorname{supp}\left(\eta_{0}\right) \subseteq[-7 / 8,7 / 8]$. For $k \in \mathbb{N}$ we set

$$
\eta_{k}(\tau)=\eta_{0}\left(\tau / 2^{k}\right)-\eta_{0}\left(\tau / 2^{k-1}\right) .
$$

We write $\eta_{\leq m}=\sum_{j=0}^{m} \eta_{j}$ for $m \in \mathbb{N}$.

Set

$$
A_{k}=\left\{\begin{array}{l}
\left\{\xi \in \mathbb{R}^{n}|| \xi \mid \lesssim 1\right\}, \quad k=0, \\
\left\{\xi \in \mathbb{R}^{n}|| \xi \mid \sim 2^{k}\right\}, \quad k \geq 1,
\end{array}\right.
$$

and for the dispersion relation $\varphi(\xi, \eta)=\xi^{3}+\xi \eta^{2}, N, L \in 2^{\mathbb{N}_{0}}$

$$
\begin{aligned}
G_{N, L} & =\left\{(\tau, \xi) \in \mathbb{R} \times \mathbb{R}^{2}|| \xi|\sim N,| \tau-\varphi(\xi) \mid \sim L\right\}, \\
G_{N, \leq L} & =\left\{(\tau, \xi) \in \mathbb{R} \times \mathbb{R}^{2}|| \xi|\sim N,| \tau-\varphi(\xi) \mid \leq L\right\} .
\end{aligned}
$$


Next, we define an $X^{s, b}$-type space for the Fourier transform of frequencylocalized space-periodic functions:

$$
\begin{aligned}
& X_{k, \lambda}=\left\{f: \mathbb{R} \times \mathbb{Z}^{n} / \lambda \rightarrow \mathbb{C} \mid\right. \\
& \left.\operatorname{supp}(f) \subseteq \mathbb{R} \times A_{k},\|f\|_{X_{k, \lambda}}=\sum_{j=0}^{\infty} 2^{j / 2}\left\|\eta_{j}(\tau-\varphi(\xi)) f(\tau, \xi)\right\|_{L_{(d \xi)_{\lambda}}^{2} L_{\tau}^{2}}<\infty\right\} .
\end{aligned}
$$

Partitioning the modulation variable through a sum over $\eta_{j}$ yields the estimate

$$
\left\|\int_{\mathbb{R}}\left|f_{k}\left(\tau^{\prime}, \xi\right)\right| d \tau^{\prime}\right\|_{L_{(d \xi)}^{2}} \lesssim\left\|f_{k}\right\|_{X_{k, \lambda}}
$$

Also, we record the estimate

$$
\begin{aligned}
& \sum_{j=l+1}^{\infty} 2^{j / 2}\left\|\eta_{j}(\tau-\varphi(\xi)) \cdot \int_{\mathbb{R}}\left|f_{k}\left(\tau^{\prime}, \xi\right)\right| \cdot 2^{-l}\left(1+2^{-l}\left|\tau-\tau^{\prime}\right|\right)^{-4} d \tau^{\prime}\right\|_{L_{(d \xi)_{\lambda}}^{2} L_{\tau}^{2}} \\
& +2^{l / 2}\left\|\eta_{\leq l}(\tau-\varphi(\xi)) \cdot \int_{\mathbb{R}}\left|f_{k}\left(\tau^{\prime}, \xi\right)\right| \cdot 2^{-l}\left(1+2^{-l}\left|\tau-\tau^{\prime}\right|\right)^{-4} d \tau^{\prime}\right\|_{L_{(d \xi)_{\lambda}}^{2} L_{\tau}^{2}} \\
\lesssim & \left\|f_{k}\right\|_{X_{k, \lambda}},
\end{aligned}
$$

which is a rescaled version of [14, Equation (3.5)].

In particular, we find for a Schwartz-function $\gamma$ for $k, l \in \mathbb{N}, t_{0} \in \mathbb{R}, f_{k} \in X_{k, \lambda}$ the estimate

$$
\left\|\mathcal{F}\left[\gamma\left(2^{l}\left(t-t_{0}\right)\right) \cdot \mathcal{F}^{-1}\left(f_{k}\right)\right]\right\|_{X_{k, \lambda}} \lesssim_{\gamma}\left\|f_{k}\right\|_{X_{k, \lambda}} .
$$

We define the spaces

$$
E_{k, \lambda}=\left\{u_{0}: \lambda \mathbb{T}^{n} \rightarrow \mathbb{C} \mid \operatorname{supp}\left(\hat{u}_{0}\right) \subseteq A_{k},\left\|u_{0}\right\|_{E_{k, \lambda}}=\left\|u_{0}\right\|_{L_{\lambda}^{2}}<\infty\right\},
$$

which are the spaces for the dyadically localized energy.

Next, we set

$$
C_{0}\left(\mathbb{R}, E_{k, \lambda}\right)=\left\{u_{k} \in C\left(\mathbb{R}, E_{k, \lambda}\right) \mid \operatorname{supp}\left(u_{k}\right) \subseteq[-4,4]\right\}
$$

and define for a frequency $2^{k}$ the following short-time $X^{s, b}$-space:

$$
F_{k, \lambda}=\left\{u_{k} \in C_{0}\left(\mathbb{R}, E_{k, \lambda}\right) \mid\left\|u_{k}\right\|_{F_{k, \lambda}}=\sup _{t_{k} \in \mathbb{R}}\left\|\mathcal{F}\left[u_{k} \eta_{0}\left(2^{k}\left(t-t_{k}\right)\right)\right]\right\|_{X_{k, \lambda}}<\infty\right\} .
$$

The frequency dependent time localization for frequencies $N \in 2^{\mathbb{N}_{0}}$ is $T(N)=N^{-1}$. This allows us to overcome the derivative loss in the nonlinear estimate (cf. [28]). Similarly, we define the spaces to capture the nonlinearity:

$$
\begin{aligned}
& N_{k, \lambda}=\left\{u_{k} \in C_{0}\left(\mathbb{R}, E_{k, \lambda}\right) \mid\right. \\
& \left.\left\|u_{k}\right\|_{N_{k, \lambda}}=\sup _{t_{k} \in \mathbb{R}}\left\|\left(\tau-\varphi(\xi)+i 2^{k}\right)^{-1} \mathcal{F}\left[u_{k} \eta_{0}\left(2^{k}\left(t-t_{k}\right)\right)\right]\right\|_{X_{k, \lambda}}<\infty\right\} .
\end{aligned}
$$

We localize the spaces in time in the usual way. For $T \in(0,1]$ we set

$$
F_{k, \lambda}(T)=\left\{u_{k} \in C\left([-T, T], E_{k, \lambda}\right) \mid\left\|u_{k}\right\|_{F_{k, \lambda}(T)}=\inf _{\tilde{u}_{k}=u_{k} \operatorname{in}[-T, T]}\left\|\tilde{u}_{k}\right\|_{F_{k, \lambda}}<\infty\right\}
$$

and

$$
N_{k, \lambda}(T)=\left\{u_{k} \in C\left([-T, T], E_{k, \lambda}\right) \mid\left\|u_{k}\right\|_{N_{k, \lambda}(T)}=\inf _{\tilde{u}_{k}=u_{k} \operatorname{in}[-T, T]}\left\|\tilde{u}_{k}\right\|_{N_{k, \lambda}}<\infty\right\} .
$$


We assemble the spaces for dyadically localized frequencies in a straight-forward manner using Littlewood-Paley theory: as an energy space for solutions we consider

$$
\begin{aligned}
& E_{\lambda}^{s}(T)=\left\{u \in C\left([-T, T], H_{\lambda}^{\infty}\right) \mid\right. \\
&\|u\|_{E_{\lambda}^{s}(T)}^{2}=\left\|P_{\leq 0} u(0)\right\|_{L_{\lambda}^{2}}^{2}+\sum_{N \in 2^{\mathbb{N}}} \sup _{k} \in[-T, T] \\
&\left.N^{2 s}\left\|P_{N} u\left(t_{k}\right)\right\|_{L_{\lambda}^{2}}^{2}<\infty\right\} .
\end{aligned}
$$

We define the short-time $X^{s, b}$-space for the solution

$$
F_{\lambda}^{s}(T)=\left\{u \in C\left([-T, T], H_{\lambda}^{\infty}\right) \mid\|u\|_{F_{\lambda}^{s}(T)}^{2}=\sum_{N=2^{n}, n \in \mathbb{N}_{0}} N^{2 s}\left\|P_{N} u\right\|_{F_{n, \lambda}(T)}^{2}<\infty\right\}
$$

and for the nonlinearity we consider

$$
N_{\lambda}^{s}(T)=\left\{u \in C\left([-T, T], H_{\lambda}^{\infty}\right) \mid\|u\|_{N_{\lambda}^{s}(T)}^{2}=\sum_{N=2^{n}, n \in \mathbb{N}_{0}} N^{2 s}\left\|P_{n} u\right\|_{N_{n, \lambda}(T)}^{2}<\infty\right\} .
$$

We also make use of $k$-acceptable time multiplication factors (cf. [15]): for $k \in \mathbb{N}_{0}$ we set

$$
S_{k}=\left\{m_{k} \in C^{\infty}(\mathbb{R}, \mathbb{R}):\left\|m_{k}\right\|_{S_{k}}=\sum_{j=0}^{10} 2^{-j k}\left\|\partial^{j} m_{k}\right\|_{L^{\infty}}<\infty\right\} .
$$

The generic example is given by time localization on a scale of $2^{-k}$, i.e., $\eta_{0}\left(2^{k} \cdot\right)$.

The estimates (cf. [15, Eq. (2.21), p. 273])

$$
\left\{\begin{aligned}
\left\|\sum_{k \geq 0} m_{k}(t) P_{2^{k}}(u)\right\|_{F_{\lambda}^{s}(T)} & \lesssim\left(\sup _{k \geq 0}\left\|m_{k}\right\|_{S_{k}}\right) \cdot\|u\|_{F_{\lambda}^{s}(T)}, \\
\left\|\sum_{k \geq 0} m_{k}(t) P_{2^{k}}(u)\right\|_{N_{\lambda}^{s}(T)} & \lesssim\left(\sup _{k \geq 0}\left\|m_{k}\right\|_{S_{k}}\right) \cdot\|u\|_{N_{\lambda}^{s}(T)}, \\
\left\|\sum_{k \geq 0} m_{k}(t) P_{2^{k}}(u)\right\|_{E_{\lambda}^{s}(T)} & \lesssim\left(\sup _{k \geq 0}\left\|m_{k}\right\|_{S_{k}}\right) \cdot\|u\|_{E_{\lambda}^{s}(T)}
\end{aligned}\right.
$$

follow from integration by parts.

From (9) follows that we can assume $F_{k, \lambda}(T)$ functions to be supported in time on an interval $\left[-T-2^{-k-10}, T+2^{-k-10}\right]$.

We record basic properties of the short-time $X^{s, b}$-spaces introduced above. The next lemma establishes the embedding $F_{\lambda}^{s}(T) \hookrightarrow C\left([0, T], H_{\lambda}^{s}\right)$.

Lemma 2.1. $\quad$ (i) We find the estimate

$$
\|u\|_{L_{t}^{\infty} L_{\lambda}^{2}} \lesssim\|u\|_{F_{k, \lambda}}
$$

to hold for any $u \in F_{k, \lambda}$ with implicit constant independent of $k$ and $\lambda$.

(ii) Suppose that $s \in \mathbb{R}, T>0$ and $u \in F_{\lambda}^{s}(T)$. Then, we find the estimate

to hold.

$$
\|u\|_{C\left([0, T], H_{\lambda}^{s}\right)} \lesssim\|u\|_{F_{\lambda}^{s}(T)}
$$

Proof. For a proof, see [15, Lemma 3.1., p. 274] in Euclidean space and $[14$, Lemma $3.2,3.3]$ in the periodic case. Independence of the period length follows from rescaling.

We state the energy estimate for the above short-time $X^{s, b}$-spaces. The proof, which is carried out on the real line in [15, Proposition 3.2., p. 274] and in the periodic case in [14, Proposition 4.1.], is omitted.

Proposition 2.2. Let $T \in(0,1], \lambda \geq 1$ and $u, v \in C\left([-T, T], H_{\lambda}^{\infty}\right)$ satisfy the equation

$$
\partial_{t} u+\left(\partial_{x_{1}}^{3}+\partial_{x_{1}} \partial_{x_{2}}^{2}\right) u=v \text { in }(-T, T) \times \lambda \mathbb{T}^{2} .
$$


Then, we find the following estimate to hold for any $s \in \mathbb{R}$ with implicit constant independent of $s, T$ and $\lambda$ :

$$
\|u\|_{F_{\lambda}^{s}(T)} \lesssim\|u\|_{E_{\lambda}^{s}(T)}+\|v\|_{N_{\lambda}^{s}(T)} .
$$

Below we have to consider the action of sharp time cutoffs in the $X_{k}$-spaces. Recall from the usual $X^{s, b}$-space-theory that multiplication with a sharp cutoff in time is not bounded. However, we find the following estimate to hold:

Lemma 2.3. [14, Lemma 3.5] Let $N=2^{n}, n \in \mathbb{N}_{0}$ and $\lambda \geq 1$. Then, for any interval $I=\left[t_{1}, t_{2}\right] \subseteq \mathbb{R}$, we find the following estimate to hold:

$$
\sup _{j \geq 0} 2^{j / 2}\left\|\eta_{j}(\tau-\varphi(\xi)) \mathcal{F}_{t, x}\left[1_{I}(t) P_{N} u\right]\right\|_{L_{\tau}^{2} L_{(d \xi)}^{2}} \lesssim\left\|\mathcal{F}_{t, x}\left(P_{N} u\right)\right\|_{X_{n, \lambda}}
$$

with implicit constant independent of $n, \lambda$ and $I$.

\section{Short-time NONLINEAR ESTIMATES}

We recall short-time nonlinear estimates on $\lambda \mathbb{T}^{2}$ from [28] for $s>1 / 2$.

Proposition 3.1. Let $\lambda \geq 1, T \in(0,1], 1 / 2<s \leq s^{\prime}$. Then, we find the following estimates to hold for $u_{1}, u_{2} \in F_{\lambda}^{s}(T)$ :

$$
\begin{aligned}
\left\|\partial_{x_{1}}\left(u_{1} u_{2}\right)\right\|_{N_{\lambda}^{s}(T)} & \lesssim\left\|u_{1}\right\|_{F_{\lambda}^{s}(T)}\left\|u_{2}\right\|_{F_{\lambda}^{s}(T)}, \\
\left\|\partial_{x_{1}}\left(u_{1} u_{2}\right)\right\|_{N_{\lambda}^{0}(T)} & \lesssim\left\|u_{1}\right\|_{F_{\lambda}^{0}(T)}\left\|u_{2}\right\|_{F_{\lambda}^{s}(T)} .
\end{aligned}
$$

Proof. The proof for $\lambda=1$ is given in [28, Prop. 7.5]; the general case follows from rescaling.

\section{Global nonlinear Loomis-Whitney inequalities}

In this section, global nonlinear Loomis-Whitney inequalities are discussed. After globalizing local results in $\mathbb{R}^{3}$, we turn to Loomis-Whitney-type inequalities on $\mathbb{R} \times \mathbb{Z}^{2} / N$. The arguments from considering Euclidean space will be useful on $\mathbb{R} \times \mathbb{Z}^{2} / N$.

4.1. Loomis-Whitney inequalities on $\mathbb{R}^{3}$. For $i=1,2,3$, letting

$S_{i}:=\left\{\left(x_{1}, x_{2}, x_{3}\right) \in \mathbb{R}^{3} \mid x_{i}=0\right\}$, the classical Loomis-Whitney inequality in $\mathbb{R}^{3}$ is described as

$$
\left\|f_{1} * f_{2}\right\|_{L^{2}\left(S_{3}\right)} \leq\left\|f_{1}\right\|_{L^{2}\left(S_{1}\right)}\left\|f_{2}\right\|_{L^{2}\left(S_{2}\right)} .
$$

Note that the case of fully transverse hyperplanes, quantified by $A$ in Assumption 1.(iii), is recovered by a change of variables, and we find the above estimate to hold with constant $A^{1 / 2}$. If $S_{1}, S_{2}, S_{3}$ are oriented hypersurfaces in $\mathbb{R}^{3}$, then the above is called the nonlinear Loomis-Whitney inequalities in $\mathbb{R}^{3}$.

The estimate for fully transverse hyperplanes was extended to $C^{3}$-hypersurfaces in [7] by Bennett-Carbery-Wright. Then, Bejenaru-Herr-Tataru relaxed the regularity conditions of the hypersurfaces in [3] by employing induction on scales. In these results, the transversality of the oriented hypersurfaces determines the constant for which the estimate from the above display holds, which matches the case of hyperplanes. The constant also depends on regularity properties of the surfaces; see Assumption 1 below. Furthermore, the results from [7, 3] are local, i.e., these are only stated for bounded hypersurfaces.

Nonlinear Loomis-Whitney inequalities yield smoothing effects in Euclidean space related to bilinear Strichartz estimates. This cannot hold on $\mathbb{R} \times \mathbb{Z}^{2} / N$, due to the 
discrete nature of the counting measure. One can well have a fully transverse interaction of three frequencies on a lattice, which cannot yield any smoothing effect. See the end of this section for an example.

Before turning to Loomis-Whitney-type inequalities on $\mathbb{R} \times \mathbb{Z}^{2} / N$, we shall see how to remove the locality assumption in Euclidean space. The underlying argument will be crucial to handle the discrete case. Our argument is related to a recent work by Koch-Steinerberger [18]. In [18, Theorem 2.1, p. 1226] a global result for hypersurfaces described as Lipschitz graphs is given. However, the stated dependence on the transversality constant is worse than in the case of hyperplanes in [18]. The argument in [18] does not make use of induction on scales, contrary to [3], but relies entirely on suitable decompositions and almost orthogonality. Our proof is also based on decompositions of the hypersurfaces and almost orthogonality. We improve the dependence on the transversality given in [18] for hypersurfaces slightly more regular than Lipschitz, which we do not cover.

In the following we consider $C^{1, \beta}$-hypersurfaces given as rotated graphs of $C^{1, \beta}$ functions following [2, Assumption 1.1].

Assumption 1. For $i=1,2,3$ there exist $0<\beta \leq 1, b>0, A \geq 1, F_{i} \in C^{1, \beta}\left(\mathcal{U}_{i}\right)$, where the $\mathcal{U}_{i}$ denote open and convex sets in $\mathbb{R}^{2}$ and $G_{i} \in O(3)$ such that

(i) the oriented surfaces $S_{i}$ are given by

$$
S_{i}=G_{i} g r\left(F_{i}\right), \quad g r\left(F_{i}\right)=\left\{(x, y, z) \in \mathbb{R}^{3} \mid z=F_{i}(x, y),(x, y) \in \mathcal{U}_{i}\right\} .
$$

(ii) the unit normal vector field $\mathfrak{n}_{i}$ on $S_{i}$ satisfies the Hölder condition

$$
\sup _{\sigma, \tilde{\sigma} \in S_{i}} \frac{\left|\mathfrak{n}_{i}(\sigma)-\mathfrak{n}_{i}(\tilde{\sigma})\right|}{|\sigma-\tilde{\sigma}|^{\beta}}+\frac{\left|\mathfrak{n}_{i}(\sigma)(\sigma-\tilde{\sigma})\right|}{|\sigma-\tilde{\sigma}|^{1+\beta}} \leq b ;
$$

(iii) the matrix $N\left(\sigma_{1}, \sigma_{2}, \sigma_{3}\right)=\left(\mathfrak{n}_{1}\left(\sigma_{1}\right), \mathfrak{n}_{2}\left(\sigma_{2}\right), \mathfrak{n}_{3}\left(\sigma_{3}\right)\right)$ satisfies the transversality condition

$$
A^{-1} \leq \operatorname{det} N\left(\sigma_{1}, \sigma_{2}, \sigma_{3}\right) \leq 1
$$

for all $\left(\sigma_{1}, \sigma_{2}, \sigma_{3}\right) \in S_{1} \times S_{2} \times S_{3}$.

Under Assumption 1, we establish the nonlinear Loomis-Whitney inequalities without locality assumptions on $S_{i}$.

Theorem 4.1. Suppose that $\left(S_{i}\right)_{i=1}^{3}$ satisfies Assumption 1. Then, for each $f \in$ $L^{2}\left(S_{1}\right)$ and $g \in L^{2}\left(S_{2}\right)$, we have

$$
\|f * g\|_{L^{2}\left(S_{3}\right)} \leq C A^{1 / 2}\|f\|_{L^{2}\left(S_{1}\right)}\|g\|_{L^{2}\left(S_{2}\right)}
$$

where the constant $C>0$ is independent of $\beta$ and $b$.

Note that in [3], though the sharp dependence on $A$ is obtained, the constant $C$ in the above display depends on $\beta$ and $b$. To begin with the proof, we see how we can quantify the overlap of thickened hypersurfaces. We write $S_{i}(\varepsilon)=$ $G_{i}\left\{(x, y, z) \in \mathcal{U}_{i} \times \mathbb{R}|| z-F_{i}(x, y) \mid<\varepsilon\right\}$ with notations from above and define $\chi_{M}$ as the characteristic function of a set $M$.

Proposition 4.2. Suppose that $\left(S_{i}\right)_{i=1}^{3}$ satisfies Assumption 1. Then, for $\varepsilon>0$, the following estimate holds true:

$$
\int_{\mathbb{R}^{3}} \chi_{S_{1}(\varepsilon)}(x) \chi_{S_{2}(\varepsilon)}(x) \chi_{S_{3}(\varepsilon)}(x) d x \lesssim A \varepsilon^{3},
$$

where the implicit constant is independent of $\beta$ and $b$. 
Proof. Clearly, by the definitions of $S_{i}(\varepsilon)$, we may assume that $\varepsilon=\varepsilon(A, \beta, b)$ is sufficiently small. We start with the elementary case that $S_{i}$ are three transverse hyperplanes $\left(H_{i}\right)_{i=1}^{3}$. The estimate

$$
\int_{\mathbb{R}^{3}} \chi_{H_{1}(\varepsilon)}(x) \chi_{H_{2}(\varepsilon)}(x) \chi_{H_{3}(\varepsilon)}(x) d x \lesssim A \varepsilon^{3}
$$

follows from a linear change of variables, mapping the normals of the hyperplanes to the unit matrix.

We turn to the nonlinear case. Let $p \in S_{1}(\varepsilon) \cap S_{2}(\varepsilon) \cap S_{3}(\varepsilon)$, as for an empty intersection there is nothing to show. We observe that

$$
\int_{\mathbb{R}^{3}} \chi_{S_{1}(\varepsilon)}(x) \chi_{S_{2}(\varepsilon)}(x) \chi_{S_{3}(\varepsilon)}(x) d x=\int_{B(100 A \varepsilon, p)} \chi_{S_{1}(\varepsilon)}(x) \chi_{S_{2}(\varepsilon)}(x) \chi_{S_{3}(\varepsilon)}(x) d x .
$$

To confine the range of integration to $B(100 A \varepsilon, p)$, suppose that there is $q \in S_{1}(\varepsilon) \cap$ $S_{2}(\varepsilon) \cap S_{3}(\varepsilon)$ with $d(p, q) \geq 100 A \varepsilon$. For $i=1,2,3$ we can find $q_{i} \in S_{i}$ with $d\left(q_{i}, q\right) \leq \varepsilon$ and $p_{i} \in S_{i}$ with $d\left(p_{i}, p\right) \leq \varepsilon$. We have $d\left(q_{i}, p_{i}\right) \geq 98 A \varepsilon$. By the mean-value theorem, we find a normal vector $\mathfrak{n}_{i}$ of $S_{i}$ with $\mathfrak{n}_{i} \perp p_{i}-q_{i}$. It is straight-forward to check that $\left|\operatorname{det}\left(\mathfrak{n}_{1}, \mathfrak{n}_{2}, \mathfrak{n}_{3}\right)\right| \ll A^{-1}$. This contradiction allows us to bound the domain of integration like above.

To reduce the nonlinear case to the case of hyperplanes, we shall approximate $S_{i}(\varepsilon) \cap B(100 \varepsilon, p)$ with $T_{p_{i}} S_{i}(C \varepsilon), p_{i} \in S_{i}, d\left(p_{i}, p\right) \leq \varepsilon$. Here, $T_{p} S$ denotes the tangent space at $S$, as a subset of $\mathbb{R}^{3}$. Observe that by the $C^{1, \beta}$-property Assumption 1.(ii), $\lambda_{i} \in S_{i}$ satisfy the estimate

$$
\left|\mathfrak{n}_{i}\left(p_{i}\right) \cdot\left(p_{i}-\lambda_{i}\right)\right| \leq b\left|p_{i}-\lambda_{i}\right|^{1+\beta} .
$$

For $\varepsilon \ll b^{-1 / \beta} A^{-(1+1 / \beta)}$, we find $T_{p_{i}} S_{i}(C \varepsilon) \supseteq B(100 A \varepsilon, p) \cap S_{i}(\varepsilon)$.

To finish the proof, we estimate by our considerations in the case of hyperplanes

$$
\int_{B(100 A \varepsilon, p)} \chi_{T_{p_{1}} S_{1}(C \varepsilon)}(x) \chi_{T_{p_{2}} S_{2}(C \varepsilon)}(x) \chi_{T_{p_{3}} S_{3}(C \varepsilon)}(x) d x \lesssim A \varepsilon^{3} .
$$

This completes the proof.

By the above proposition, we show the following global nonlinear Loomis-Whitney inequality for thickened hypersurfaces. This will allow us to remove the locality assumption from [3] by taking the thickness to zero in the next subsection.

Theorem 4.3. Let $A$ be dyadic and $f_{i} \in L^{2}\left(S_{i}(\varepsilon)\right), i=1,2$. Suppose that $\left(S_{i}\right)_{i=1}^{3}$ satisfies Assumption 1. Then, for $\varepsilon>0$ we find the following estimate to hold

$$
\left\|f_{1} * f_{2}\right\|_{L^{2}\left(S_{3}(\varepsilon)\right)} \lesssim \varepsilon^{3 / 2} A^{1 / 2}\left\|f_{1}\right\|_{L^{2}\left(S_{1}(\varepsilon)\right)}\left\|f_{2}\right\|_{L^{2}\left(S_{2}(\varepsilon)\right)},
$$

where the implicit constant is independent of $\beta$ and $b$.

Proof. Let $\left\{B_{\varepsilon, j}\right\}_{j \in \mathbb{N}}$ denote a finitely overlapping family of balls with radius $\varepsilon$ covering $\mathbb{R}^{3}$. Set $J_{3, \varepsilon}=\left\{j \in \mathbb{N} \mid B_{\varepsilon, j} \cap S_{3}(\varepsilon) \neq \emptyset\right\}$ and $S_{3, j}(\varepsilon)=B_{\varepsilon, j} \cap S_{3}(\varepsilon)$. We break up the support of $f_{3}$ into $\left\{S_{3, j}(\varepsilon)\right\}_{j \in J_{3, \varepsilon}}$ to get

$$
\left|\int_{\mathbb{R}^{3}}\left(f_{1} * f_{2}\right)(\lambda) f_{3}(\lambda) d \lambda\right| \leq \sum_{j \in J_{3, \varepsilon}}\left|\int_{\mathbb{R}^{3}}\left(f_{1} * f_{2}\right)(\lambda) f_{3}\right|_{S_{3, j}(\varepsilon)}(\lambda) d \lambda \mid .
$$

We use the Cauchy-Schwarz inequality to estimate the single contributions

$$
\left|\int_{\mathbb{R}^{3}}\left(f_{1} * f_{2}\right)(\lambda) f_{3}\right|_{S_{3, j}(\varepsilon)}(\lambda) d \lambda \mid \lesssim \varepsilon^{3 / 2}\left\|f_{1}\right\|_{L^{2}\left(S_{1, j, \varepsilon}\right)}\left\|f_{2}\right\|_{L^{2}\left(S_{2, j, \varepsilon}\right)}\left\|f_{3}\right\|_{L^{2}\left(S_{3, j}(\varepsilon)\right)},
$$


where

$$
\begin{aligned}
& S_{1, j, \varepsilon}=\left\{\lambda_{1} \in S_{1}(\varepsilon) \mid \exists \lambda^{\prime} \in B_{\varepsilon, j}: \lambda^{\prime}-\lambda_{1} \in S_{2}(\varepsilon)\right\}, \\
& S_{2, j, \varepsilon}=\left\{\lambda_{2} \in S_{2}(\varepsilon) \mid \exists \lambda^{\prime} \in B_{\varepsilon, j}: \lambda^{\prime}-\lambda_{2} \in S_{1}(\varepsilon)\right\} .
\end{aligned}
$$

By the Cauchy-Schwarz inequality, for all $\left(\lambda_{1}, \lambda_{2}\right) \in S_{1}(\varepsilon) \times S_{2}(\varepsilon)$, it suffices to show

$$
\sum_{j \in J_{3, \varepsilon}} \chi_{S_{1, j, \varepsilon} \times S_{2, j, \varepsilon}}\left(\lambda_{1}, \lambda_{2}\right) \lesssim A
$$

Indeed, assuming (16), we conclude

$$
\begin{aligned}
& \sum_{j}\left|\int_{\mathbb{R}^{3}}\left(f_{1} * f_{2}\right)(\lambda) f_{3}\right|_{S_{3, j}(\varepsilon)}(\lambda) d \lambda \mid \\
\lesssim & \varepsilon^{3 / 2} \sum_{j}\left\|f_{1}\right\|_{L^{2}\left(S_{1, j, \varepsilon}\right)}\left\|f_{2}\right\|_{L^{2}\left(S_{2, j, \varepsilon}\right)}\left\|f_{3}\right\|_{L^{2}\left(S_{3, j}(\varepsilon)\right)} \\
\lesssim & \varepsilon^{3 / 2}\left(\sum_{j}\left\|f_{3}\right\|_{L^{2}\left(S_{3, j}(\varepsilon)\right)}^{2 / 2}\left(\sum_{j}\left\|f_{1}\right\|_{L^{2}\left(S_{1, j, \varepsilon}\right)}^{2}\left\|f_{2}\right\|_{L^{2}\left(S_{2, j, \varepsilon}\right)}^{2}\right)^{1 / 2}\right. \\
\lesssim & \left.\varepsilon^{3 / 2}\left\|f_{3}\right\|_{L^{2}\left(S_{3}(\varepsilon)\right)}\left(\sum_{j}\left\|f_{1} \chi_{S_{1, j, \varepsilon}}\right\|_{L^{2}}^{2}\left\|f_{2} \chi_{S_{2, j, \varepsilon}}\right\|_{L^{2}}^{2}\right)^{1 / 2}\right)^{1 / 2}\left(\varepsilon^{3 / 2}\left\|f_{3}\right\|_{L^{2}\left(S_{3}(\varepsilon)\right)}\left(\sum_{\mathbb{R}^{3} \times \mathbb{R}^{3}} \sum_{j} \chi_{S_{1, j, \varepsilon} \times S_{2, j, \varepsilon}}\left(\lambda_{1}, \lambda_{2}\right)\left|f_{1}\left(\lambda_{1}\right)\right|^{2}\left|f_{2}\left(\lambda_{2}\right)\right|^{2} d \lambda_{1} d \lambda_{2}\right)^{1 / 2}\right. \\
\lesssim & \varepsilon^{3 / 2} A^{1 / 2} \prod_{i=1}^{3}\left\|f_{i}\right\|_{L^{2}\left(S_{i}(\varepsilon)\right)} .
\end{aligned}
$$

Thus, the remainder of the proof is devoted to the proof of (16).

Without loss of generality, we may assume that there exists $j_{0} \in J_{3, \varepsilon}$ such that $\left(\lambda_{1}, \lambda_{2}\right) \in S_{1, j_{0}, \varepsilon} \times S_{2, j_{0}, \varepsilon}$. Suppose that $j \in J_{3, \varepsilon}$ satisfies $\left(\lambda_{1}, \lambda_{2}\right) \in S_{1, j, \varepsilon} \times S_{2, j, \varepsilon}$. We define $\lambda_{j_{0}} \in B_{\varepsilon, j_{0}}$ as the center of $B_{\varepsilon, j_{0}}$ and choose $\lambda_{j} \in B_{\varepsilon, j}$ arbitrarily. The assumption $\lambda_{1} \in S_{1, j_{0}, \varepsilon} \cap S_{1, j, \varepsilon}$ implies that there exist $\lambda_{j_{0}}^{\prime} \in B_{\varepsilon, j_{0}}$ and $\lambda_{j}^{\prime} \in B_{\varepsilon, j}$ such that $\left(\lambda_{j_{0}}^{\prime}-\lambda_{1}\right) \in S_{2}(\varepsilon)$ and $\left(\lambda_{j}^{\prime}-\lambda_{1}\right) \in S_{2}(\varepsilon)$. Similarly, the assumption $\lambda_{2} \in S_{2, j_{0}, \varepsilon} \cap S_{2, j, \varepsilon}$ yields $\tilde{\lambda}_{j_{0}} \in B_{\varepsilon, j_{0}}$ and $\tilde{\lambda}_{j} \in B_{\varepsilon, j}$ such that $\left(\tilde{\lambda}_{j_{0}}-\lambda_{2}\right) \in S_{1}(\varepsilon)$ and $\left(\tilde{\lambda}_{j}-\lambda_{2}\right) \in S_{1}(\varepsilon)$. We note that

$$
\begin{aligned}
& \left|\left(\lambda_{j_{0}}^{\prime}-\lambda_{1}\right)-\left(\lambda_{j}^{\prime}-\lambda_{1}\right)-\left(\lambda_{j_{0}}-\lambda_{j}\right)\right| \leq 4 \varepsilon, \\
& \left|\left(\tilde{\lambda}_{j_{0}}-\lambda_{2}\right)-\left(\tilde{\lambda}_{j}-\lambda_{2}\right)-\left(\lambda_{j_{0}}-\lambda_{j}\right)\right| \leq 4 \varepsilon .
\end{aligned}
$$

Now we define the new hypersurfaces $S_{1}^{\prime}=S_{1}^{\prime}\left(j_{0}, \lambda_{2}\right)$ and $S_{2}^{\prime}=S_{2}^{\prime}\left(j_{0}, \lambda_{1}\right)$ as

$$
S_{1}^{\prime}=S_{1}-\left(\tilde{\lambda}_{j_{0}}-\lambda_{2}\right)+\lambda_{j_{0}}, \quad S_{2}^{\prime}=S_{2}-\left(\lambda_{j_{0}}^{\prime}-\lambda_{1}\right)+\lambda_{j_{0}} .
$$

Since $\left(\tilde{\lambda}_{j_{0}}-\lambda_{2}\right) \in S_{1}(\varepsilon)$ and $\left(\lambda_{j_{0}}^{\prime}-\lambda_{1}\right) \in S_{2}(\varepsilon)$, it follows that $\lambda_{j_{0}} \in S_{1}^{\prime}(\varepsilon) \cap S_{2}^{\prime}(\varepsilon)$. In addition, we deduce from $\left(\tilde{\lambda}_{j}-\lambda_{2}\right) \in S_{1}(\varepsilon)$, (18) and $\left(\lambda_{j}^{\prime}-\lambda_{1}\right) \in S_{2}(\varepsilon)$, (17) that

$$
\operatorname{dist}\left(\lambda_{j}, S_{1}^{\prime}\right) \leq 6 \varepsilon, \quad \operatorname{dist}\left(\lambda_{j}, S_{2}^{\prime}\right) \leq 6 \varepsilon .
$$


Since $\lambda_{j} \in B_{\varepsilon, j}$ was chosen arbitrarily, the above display implies that if $j \in J_{3, \varepsilon}$ satisfies $\left(\lambda_{1}, \lambda_{2}\right) \in S_{1, j, \varepsilon} \times S_{2, j, \varepsilon}$, then it holds that

$$
B_{\varepsilon, j} \subseteq S_{1}^{\prime}(6 \varepsilon) \cap S_{2}^{\prime}(6 \varepsilon) \cap S_{3}(6 \varepsilon) .
$$

Consequently, (16) follows from Proposition 4.2 as

$$
\int_{\mathbb{R}^{3}} \chi_{S_{1}^{\prime}(6 \varepsilon)} \chi_{S_{2}^{\prime}(6 \varepsilon)} \chi_{S_{3}(6 \varepsilon)} \lesssim \varepsilon^{3} A
$$

4.2. Functions on thickened hypersurfaces. With the notations from above and $\left(f_{i}\right)_{i=1}^{3} \subseteq C_{c}\left(\mathbb{R}^{3}\right)$ compactly supported functions that

(19)

$\left.\left.\frac{1}{(2 \varepsilon)^{3}} \int_{\mathbb{R}^{3}}\left(\left.\left.f_{1}\right|_{S_{1}(\varepsilon)} * f_{2}\right|_{S_{2}(\varepsilon)}\right)(x) f_{3}\right|_{S_{3}(\varepsilon)}(x) d x \rightarrow \int_{S_{3}}\left(\left.\left.f_{1}\right|_{S_{1}} * f_{2}\right|_{S_{2}}\right)(x) f_{3}\right|_{S_{3}}(x) d \sigma_{3}(x)$,

where $\sigma_{3}$ denotes the surface measure on $S_{3}$. Since $\varepsilon^{-1 / 2}\left\|f_{i}\right\|_{L^{2}\left(S_{i}(\varepsilon)\right)} \rightarrow\left\|f_{i}\right\|_{L^{2}\left(S_{i}\right)}$, the estimate (19), together with Theorem 4.3 immediately yields Theorem 4.1.

At several points, we make use of the coarea formula:

Theorem 4.4 (Coarea formula). Let $\Omega \subseteq \mathbb{R}^{n}$ be an open set and $u: \Omega \rightarrow \mathbb{R}^{k} a$ Lipschitz-continuous mapping, where $k \leq n$. Then, the following equality holds:

$$
\int_{\Omega} g(x) d x=\int_{\mathbb{R}^{k}} \int_{u^{-1}(t)} g(x)\left|J_{k}(x)\right| d \mathcal{H}^{k}(x) d t,
$$

where $d \mathcal{H}^{k}$ denotes $k$-dimensional Hausdorff measure and $J_{k}(x)=\left|\operatorname{det}\left((J u)^{t} J u\right)\right|^{1 / 2}$ the $k$-Jacobian of $u$.

We have the following lemma on the convolution on hypersurfaces:

Lemma 4.5. Let $0<\beta \leq 1$ and $S_{1}, S_{2}$ denote oriented transverse $C^{1, \beta}$-hypersurfaces and let $f_{i} \in C_{c}\left(\mathbb{R}^{3}\right), i=1,2$. Then, the following holds true:

$$
\left.\sup _{x \in \mathbb{R}^{3}}\left|\frac{1}{(2 \varepsilon)^{2}} f_{1}\right|_{S_{1}(\varepsilon)} * f_{2}\right|_{S_{2}(\varepsilon)}(x)-\left.\left.f_{1}\right|_{S_{1}} * f_{2}\right|_{S_{2}}(x) \mid \rightarrow 0 \text { as } \varepsilon \rightarrow 0 .
$$

Proof. Let $F_{1}$ parametrize $S_{1}$ and $F_{2}^{x}$ parametrize $x-S_{2}$. In the first step, we use the coarea formula with $u=\left(F_{1}, F_{2}^{x}\right)$ to decompose $S_{1}(\varepsilon)$ and $S_{2}(\varepsilon)$ into hypersurfaces:

$$
\begin{aligned}
& \left.\left.f_{1}\right|_{S_{1}(\varepsilon)} * f_{2}\right|_{S_{2}(\varepsilon)}(x) \\
= & \int_{\substack{y \in S_{1}(\varepsilon), x-y \in S_{2}(\varepsilon)}} f_{1}(x-y) f_{2}(y) d y \\
= & \int_{-\varepsilon}^{\varepsilon} \int_{-\varepsilon}^{\varepsilon} \int_{\substack{x-y \in S_{2}^{\varepsilon_{2}} \\
y \in S_{1}^{\varepsilon_{1}}}} f_{1}(x-y) f_{2}(y) \sin ^{-1}(\alpha(y, x-y)) d \mathcal{H}^{1}(y) d \varepsilon_{1} d \varepsilon_{2} .
\end{aligned}
$$

Here, $\alpha(y, x-y)$ denotes the angle between $\mathfrak{n}_{1}(y)$ and $\mathfrak{n}_{2}(x-y)$ and $\mathcal{H}^{1}$ the onedimensional Hausdorff measure. We parametrize $S_{1}^{\varepsilon_{2}} \cap\left(x-S_{2}^{\varepsilon_{1}}\right)$ by $\gamma_{x}^{\varepsilon_{1}, \varepsilon_{2}}:(0,1)=$ $I \rightarrow S_{2}^{\varepsilon_{2}} \cap\left(x-S_{1}^{\varepsilon_{1}}\right)$ by virtue of the implicit function theorem.

Note that $\left|S_{1}^{\varepsilon_{2}} \cap\left(x-S_{2}^{\varepsilon_{1}}\right)\right|$ depends continuously on $\varepsilon_{1}, \varepsilon_{2}$ and $x$. Moreover, it is enough to consider $S_{1} \cap\left(x-S_{2}\right) \neq \emptyset$. For these points, the implicit function theorem gives that $\gamma_{x}^{\varepsilon_{1}, \varepsilon_{2}}$ depends jointly continuously differentiable on $x, \varepsilon_{1}$ and $\varepsilon_{2}$. 
This gives by the mean value theorem

$$
\begin{aligned}
& \left.\left.f_{1}\right|_{S_{1}(\varepsilon)} * f_{2}\right|_{S_{2}(\varepsilon)}(x) \\
= & \int_{-\varepsilon}^{\varepsilon} \int_{-\varepsilon}^{\varepsilon} \int_{\substack{y \in S_{2}^{\varepsilon_{2}}, \varepsilon^{\prime} \\
x-y \in S_{1}^{\varepsilon_{1}}}} f_{1}(x-y) f_{2}(y) \sin ^{-1}(\alpha(x-y, y)) d \mathcal{H}^{1}(y) d \varepsilon_{1} d \varepsilon_{2} \\
= & \int_{-\varepsilon}^{\varepsilon} \int_{-\varepsilon}^{\varepsilon} \int_{I} f_{1}\left(x-\gamma_{x}^{\varepsilon_{1}, \varepsilon_{2}}(t)\right) f_{2}\left(\gamma_{x}^{\varepsilon_{1}, \varepsilon_{2}}(t)\right) \sin ^{-1}(\alpha)\left|\dot{\gamma}_{x}^{\varepsilon_{1}, \varepsilon_{2}}(t)\right| d t d \varepsilon_{1} d \varepsilon_{2} \\
= & (2 \varepsilon)^{2} \int_{I} f_{1}\left(x-\gamma_{x}^{\varepsilon_{1}^{\prime}, \varepsilon_{2}^{\prime}}(t)\right) f_{2}\left(\gamma_{x}^{\varepsilon_{1}, \varepsilon_{2}}(t)\right) \sin ^{-1}(\alpha)\left|\dot{\gamma}_{x}^{\varepsilon_{1}^{\prime}, \varepsilon_{2}^{\prime}}(t)\right| d t
\end{aligned}
$$

for $\varepsilon_{1}^{\prime}, \varepsilon_{2}^{\prime} \in[-\varepsilon, \varepsilon]$. The proof is complete.

Furthermore, we have the following lemma:

Lemma 4.6. Let $0<\beta \leq 1, S_{3}$ be a $C^{1, \beta}$-hypersurface and $f_{3} \in C_{c}\left(\mathbb{R}^{3}\right)$. Let

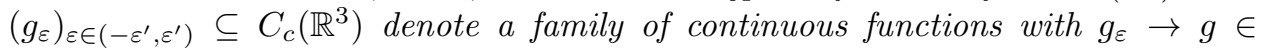
$C_{c}\left(\mathbb{R}^{3}\right)$ as $\varepsilon \rightarrow 0$. Then, we find the following estimate to hold:

$$
\left|\frac{1}{2 \varepsilon} \int_{\mathbb{R}^{3}} g_{\varepsilon}(x) f_{3}\right|_{S_{3}(\varepsilon)}(x) d x-\left.\int_{S_{3}} g(x) f_{3}\right|_{S_{3}}(x) d \sigma_{3}(x) \mid \rightarrow 0 .
$$

Proof. We use the coarea formula as in the proof of the previous lemma to write

$$
\frac{1}{2 \varepsilon} \int_{S_{3}(\varepsilon)} g_{\varepsilon}(x) f_{3}(x) d x=\frac{1}{2 \varepsilon} \int_{-\varepsilon}^{\varepsilon} \int_{S_{3}^{\varepsilon^{\prime}}} g_{\varepsilon}(x) f_{3}(x) d \mathcal{H}^{2}(x) d \varepsilon^{\prime}
$$

where $S_{3}(\varepsilon)=\bigcup_{\varepsilon^{\prime} \in[-\varepsilon, \varepsilon]} S_{3}^{\varepsilon^{\prime}}$.

By continuity of the integral in $\varepsilon^{\prime}$, we can write by the mean value theorem

$$
\frac{1}{2 \varepsilon} \int_{S_{3}(\varepsilon)} g_{\varepsilon}(x) f_{3}(x) d x=\int_{S_{3}^{\varepsilon_{3}}} g_{\varepsilon}(x) f_{3}(x) d \sigma_{3}^{\varepsilon_{3}}(x) \text { for } \varepsilon_{3} \in[-\varepsilon, \varepsilon] .
$$

Next, we choose parametrizations of $S_{3}^{\varepsilon_{3}}$ to write

$$
\int_{S_{3}^{\varepsilon_{3}}} g_{\varepsilon}(x) f_{3}(x) d \sigma_{3}^{\varepsilon_{3}}(x)=\int_{\mathbb{R}^{2}} g_{\varepsilon}\left(\psi_{\varepsilon_{3}}(x)\right) f_{3}\left(\psi_{\varepsilon_{3}}(x)\right) \sqrt{\operatorname{det}\left(\left(J \psi_{\varepsilon_{3}}\right)^{\tau}\left(J \psi_{\varepsilon_{3}}\right)\right.} d x
$$

with $J \psi_{\varepsilon}$ independent of $\varepsilon$, which is possible as varying $\varepsilon$ in $S_{3}^{\varepsilon}$ only amounts to a linear shift. The proof is complete.

Taking the above two lemmas together finishes the proof of (19).

We highlight that versions for thickened hypersurfaces like provided by Theorem 4.3 are more natural for applications in the context of dispersive equations, see Section 5, than the counterparts for actual hypersurfaces. We give another example of relevance for applications to dispersive equations, which shows that it is not enough to require the transversality at vectors respecting the convolution structure. This partially answers Question 2.2 (1) from the work [18] by Koch-Steinerberger negatively:

Proposition 4.7. There exist $C^{2}$-hypersurfaces $S_{i} \subseteq \mathbb{R}^{3}, i=1,2,3$, which satisfy

$$
\inf _{\substack{\lambda_{i} \in S_{i}, \lambda_{1}+\lambda_{2}=\lambda_{3}}}\left|\operatorname{det}\left(\mathfrak{n}_{1}\left(\lambda_{1}\right), \mathfrak{n}_{2}\left(\lambda_{2}\right), \mathfrak{n}_{3}\left(\lambda_{3}\right)\right)\right| \geq 1 / 2,
$$

and for any $C>0$, there exist $f_{i} \in L^{2}\left(S_{i}\right), i=1,2$, such that

$$
\left\|f_{1} * f_{2}\right\|_{L^{2}\left(S_{3}\right)} \geq C\left\|f_{1}\right\|_{L^{2}\left(S_{1}\right)}\left\|f_{2}\right\|_{L^{2}\left(S_{2}\right)} \text {. }
$$


Proof. For the sake of contradiction, suppose that for all $C^{2}$-hypersurfaces satisfying (21), there exists $C=C\left(S_{1}, S_{2}, S_{3}\right)>0$ such that

$$
\left\|f_{1} * f_{2}\right\|_{L^{2}\left(S_{3}\right)} \leq C\left\|f_{1}\right\|_{L^{2}\left(S_{1}\right)}\left\|f_{2}\right\|_{L^{2}\left(S_{2}\right)} .
$$

Let $-2^{-5}<c_{i}<2^{-5}$ and define three families of hypersurfaces $S_{i}^{c_{i}} \subseteq \mathbb{R}^{3}$ as follows:

$$
\begin{aligned}
& S_{1}^{c_{1}}=\left\{(x, y, z) \in \mathbb{R}^{3}|| y \mid<2^{-5}, z=c_{1}\right\}, \\
& S_{2}^{c_{2}}=\left\{(x, y, z) \in \mathbb{R}^{3}\left|y=c_{2},\right| z \mid<2^{-5}\right\}, \\
& S_{3}^{c_{3}}=\left\{(x, y, z) \in \mathbb{R}^{3} \mid z=\sin (\pi x)+c_{3}\right\} .
\end{aligned}
$$

Since $\left|c_{i}\right|<2^{-5}$, it is straight-forward to check that

$$
\inf _{\substack{0 \leq\left|c_{i}\right|<2^{-5} \\ i=1,2,3}} \inf _{\substack{\lambda_{i} \in S_{i}^{c_{i}}, \lambda_{1}+\lambda_{2}=\lambda_{3}}}\left|\operatorname{det}\left(\mathfrak{n}_{1}\left(\lambda_{1}\right), \mathfrak{n}_{2}\left(\lambda_{2}\right), \mathfrak{n}_{3}\left(\lambda_{3}\right)\right)\right| \geq 1 / 2 .
$$

By (22), for any $0 \leq\left|c_{i}\right|<2^{-5}, i=1,2,3$, we get

$$
\left\|f_{1} * f_{2}\right\|_{L^{2}\left(S_{3}^{c_{3}}\right)} \lesssim\left\|f_{1}\right\|_{L^{2}\left(S_{1}^{c_{1}}\right)}\left\|f_{2}\right\|_{L^{2}\left(S_{2}^{c_{2}}\right)}
$$

Setting $S_{i}\left(2^{-5}\right)=\bigcup_{0 \leq\left|c_{i}\right|<2^{-5}} S_{i}^{c_{i}} \subseteq \mathbb{R}^{3}$, this gives

$$
\left|\int_{\mathbb{R}^{3}}\left(\left.\left.f_{1}\right|_{S_{1}\left(2^{-5}\right)} * f_{2}\right|_{S_{2}\left(2^{-5}\right)}\right)(x) f_{3}\right|_{S_{3}\left(2^{-5}\right)}(x) d x \mid \lesssim \prod_{i=1}^{3}\left\|f_{i}\right\|_{L^{2}\left(S_{i}\left(2^{-5}\right)\right)} .
$$

For $R \gg 1$, consider

$$
\mathcal{T}_{R}=\bigcup_{\substack{|k| \leq R, k \in \mathbb{Z}}} B\left((k, 0,0), 2^{-10}\right) \subseteq S_{1}\left(2^{-5}\right) \cap S_{2}\left(2^{-5}\right) \cap S_{3}\left(2^{-5}\right) .
$$

Set $f_{1}=f_{2}=f_{3}=\chi_{\mathcal{T}_{R}}$. Then, $\left\|f_{i}\right\|_{L^{2}} \sim R^{1 / 2}$, and

$$
\left|\int_{\mathbb{R}^{3}}\left(\left.\left.f_{1}\right|_{S_{1}\left(2^{-5}\right)} * f_{2}\right|_{S_{2}\left(2^{-5}\right)}\right)(x) f_{3}\right|_{S_{3}\left(2^{-5}\right)}(x) d x \mid \sim R^{2},
$$

which contradicts (23). The proof is complete.

4.3. Loomis-Whitney-type inequalities on $\mathbb{R} \times \mathbb{Z}^{2} / N$. The previous considerations allow us to prove a version of the nonlinear Loomis-Whitney inequality on $\mathbb{R} \times$ lattices under scalable assumptions:

Proposition 4.8. Let $1 \leq A \ll N$ be dyadic and $f_{i}: \mathbb{R} \times \mathbb{Z}^{2} / N \rightarrow \mathbb{R}$. For $i=1,2,3$, let $S_{i}=\left\{\left(\psi_{i}(\xi), \xi\right) \mid \xi \in \mathbb{R}^{2}\right\}$ be hypersurfaces with $C^{1,1}$-functions $\psi_{i}$ on $\mathbb{R}^{2}$. Suppose that $\left\|\nabla \psi_{i}\right\|_{L^{\infty}} \lesssim 1$, and that the $S_{i}, i=1,2,3$ obey (12) with $\beta=1$ and (13).

Suppose that

$$
\operatorname{supp}\left(f_{i}\right) \subseteq S_{i}\left(L_{i}\right), S_{i}\left(L_{i}\right)=\left\{(\tau, k)=\left(\tau, k_{1}, k_{2}\right) \in \mathbb{R} \times \mathbb{Z}^{2} / N|| \tau-\psi_{i}(k) \mid \leq L_{i}\right\} .
$$

Then, we find the following estimate to hold:

$$
N^{4}\left|\int_{\mathbb{R} \times\left(\mathbb{Z}^{2} / N\right)}\left(f_{1} * f_{2}\right) f_{3} d \tau(d k)_{N}\right| \lesssim C\left(A, N, L_{1}, L_{2}, L_{3}\right) \prod_{i=1}^{3}\left(N\left\|f_{i}\right\|_{L_{\tau}^{2} L_{(d k)_{N}}^{2}}\right),
$$

where $^{1}$

$$
C\left(A, N, L_{1}, L_{2}, L_{3}\right)=L_{\min }^{1 / 2}\left\langle N L_{\text {med }}\right\rangle^{1 / 2}\left\langle A N L_{\max }\right\rangle^{1 / 2}
$$

\footnotetext{
${ }^{1}$ Note that the convolution on $\mathbb{Z}^{2} / N$ also carries the renormalized counting measure.
} 
We point out how in the limiting cases $N \rightarrow \infty$ or $L_{\text {med }} \rightarrow \infty$ Proposition 4.8 recovers (15) in Theorem 4.3.

Proof. The claim is that (24) holds with

$$
C\left(A, N, L_{1}, L_{2}, L_{3}\right)= \begin{cases}L_{\min }^{1 / 2}\left\langle A N L_{\max }\right\rangle^{1 / 2}, & L_{\text {med }} \leq N^{-1} \\ \left(A L_{1} L_{2} L_{3}\right)^{1 / 2} N, & N^{-1} \leq L_{\text {med }}\end{cases}
$$

Without loss of generality, we can assume $L_{1} \leq L_{2} \leq L_{3}$. If $L_{\max } \geq \frac{1}{A N}$, by decomposing $S_{3}\left(L_{3}\right)$ into $L_{3} / L_{2}$ translated $L_{2}$-thickened $S_{3}$, we can also assume that $L_{2}=L_{3}$. Furthermore, if $L_{2} \geq N^{-1}$, we decompose $S_{2}\left(L_{2}\right)$ and $S_{3}\left(L_{2}\right)$ into $N L_{2}$ translated $N^{-1}$-thickened hypersurfaces $S_{2}$ and $S_{3}$, respectively. If $L_{\max } \leq \frac{1}{A N}$, we do not decompose.

It suffices to show

$$
N^{4}\left|\int_{\mathbb{R} \times(\mathbb{Z} / N)^{2}}\left(f_{1} * f_{2}\right) f_{3} d \tau(d k)_{N}\right| \lesssim L_{1}^{1 / 2}\left\langle A N L_{2}\right\rangle^{1 / 2} \prod_{i=1}^{3}\left(N\left\|f_{i}\right\|_{L_{\tau}^{2} L_{(d k)_{N}}^{2}}\right),
$$

for $L_{2}=L_{3}$ and $L_{2} \leq N^{-1}$. The support of spatial frequencies for $f$ will be denoted by $\operatorname{supp}_{k}(f)$. Suppose that $k_{3} \in \operatorname{supp}_{k}\left(f_{3}\right)$ is fixed and define

$$
\begin{aligned}
& \Phi_{1}\left(k_{1}, \tau_{1}, k_{3}, \tau_{3}\right)=\left|\tau_{1}-\psi_{1}\left(k_{1}\right)\right|+\left|\tau_{3}-\tau_{1}-\psi_{2}\left(k_{3}-k_{1}\right)\right|+\left|\tau_{3}-\psi_{3}\left(k_{3}\right)\right|, \\
& \Phi_{2}\left(k_{2}, \tau_{2}, k_{3}, \tau_{3}\right)=\left|\tau_{2}-\psi_{2}\left(k_{2}\right)\right|+\left|\tau_{3}-\tau_{2}-\psi_{1}\left(k_{3}-k_{2}\right)\right|+\left|\tau_{3}-\psi_{3}\left(k_{3}\right)\right|, \\
& S_{k_{3}, L_{2}}^{1}=\left\{k_{1} \in \operatorname{supp}_{k} f_{1} \mid k_{3}-k_{1} \in \operatorname{supp}_{k} f_{2}\right. \text {, } \\
& \left.\exists \tau_{1}, \tau_{3} \in \mathbb{R}: \Phi_{1}\left(k_{1}, \tau_{1}, k_{3}, \tau_{3}\right) \lesssim L_{2}\right\}, \\
& S_{k_{3}, L_{2}}^{2}=\left\{k_{2} \in \operatorname{supp}_{k} f_{2} \mid k_{3}-k_{2} \in \operatorname{supp}_{k} f_{1}\right. \text {, } \\
& \left.\exists \tau_{2}, \tau_{3} \in \mathbb{R}: \Phi_{2}\left(k_{2}, \tau_{2}, k_{3}, \tau_{3}\right) \lesssim L_{2}\right\} \text {. }
\end{aligned}
$$

Note that $k_{3}-S_{k_{3}, L_{2}}^{1}=S_{k_{3}, L_{2}}^{2}$. For all fixed $\left(k_{1}, k_{2}\right) \in \operatorname{supp}_{k} f_{1} \times \operatorname{supp}_{k} f_{2}$, we show the following:

$$
\sum_{k_{3}} \chi_{S_{k_{3}, L_{2}}^{1} \times S_{k_{3}, L_{2}}^{2}}\left(k_{1}, k_{2}\right) \lesssim\left\langle A N L_{2}\right\rangle .
$$

Firstly, we consider the easy case of large $N$. Observe that for $N^{-1} \ll A^{-2}$, $N^{-1} \leq L_{\text {med }}$ this is a consequence of the considerations from the proof of Theorem 4.3 as the $1 / N$-lattice points can be related with the $\varepsilon$-balls from above.

In this case, like in (16),

$$
\sum_{k_{3}} \chi_{S_{k_{3}, L_{2}}^{1} \times S_{k_{3}, L_{2}}^{2}} \lesssim A
$$

and we infer the bound with $C\left(A, N, L_{1}, L_{2}, L_{3}\right)=\left(A L_{1} L_{2} L_{3}\right)^{1 / 2} N$.

The case of smaller $N$ requires more sophisticated arguments. We prove (26) by contradiction. First we consider the simple case $L_{2} \lesssim A^{-1} N^{-1}$. Assume that there exist $k_{3}^{\prime}, \tilde{k}_{3} \in \operatorname{supp}_{k} f_{3}$ such that $\left|k_{3}^{\prime}-\tilde{k_{3}}\right| \gg N^{-1}$ and there exists $\left(k_{1}^{\prime}, k_{2}^{\prime}\right) \in$ $\operatorname{supp}_{k} f_{1} \times \operatorname{supp}_{k} f_{2}$ such that

$$
\left(k_{1}^{\prime}, k_{2}^{\prime}\right) \in\left(S_{k_{3}^{\prime}, L_{2}}^{1} \times S_{k_{3}^{\prime}, L_{2}}^{2}\right) \cap\left(S_{\widetilde{k_{3}}, L_{2}}^{1} \times S_{\widetilde{k_{3}, L_{2}}}^{2}\right) .
$$

For $k_{i} \in \operatorname{supp}_{k} f_{i}$, let us write $\lambda_{i}\left(k_{i}\right)=\left(k_{i}, \psi_{i}\left(k_{i}\right)\right) \in S_{i}$ for $k_{i} \in \mathbb{Z}^{2} / N$. Then $k_{1}^{\prime} \in S_{k_{3}^{\prime}, L_{2}}^{1}$ implies that

$$
\left|\lambda_{1}\left(k_{1}^{\prime}\right)+\lambda_{2}^{\prime}\left(k_{3}^{\prime}-k_{1}^{\prime}\right)-\lambda_{3}\left(k_{3}^{\prime}\right)\right| \lesssim A^{-1} N^{-1} .
$$


Similarly, it follows from (27) that

$$
\begin{aligned}
& \left|\lambda_{1}\left(k_{3}^{\prime}-k_{2}^{\prime}\right)+\lambda_{2}\left(k_{2}^{\prime}\right)-\lambda_{3}\left(k_{3}^{\prime}\right)\right| \lesssim A^{-1} N^{-1}, \\
& \left|\lambda_{1}\left(k_{1}^{\prime}\right)+\lambda_{2}\left(\tilde{k_{3}}-k_{1}^{\prime}\right)-\lambda_{3}\left(\tilde{k_{3}}\right)\right| \lesssim A^{-1} N^{-1}, \\
& \left|\lambda_{1}\left(\tilde{k_{3}}-k_{2}^{\prime}\right)+\lambda_{2}\left(k_{2}^{\prime}\right)-\lambda_{3}\left(\tilde{k_{3}}\right)\right| \lesssim A^{-1} N^{-1} .
\end{aligned}
$$

(28)-(31) yield

$$
\begin{aligned}
& \left|\left(\lambda_{1}\left(k_{3}^{\prime}-k_{2}^{\prime}\right)-\lambda_{1}\left(\tilde{k_{3}}-k_{2}^{\prime}\right)\right)-\left(\lambda_{3}\left(k_{3}^{\prime}\right)-\lambda_{3}\left(\tilde{k_{3}}\right)\right)\right| \lesssim A^{-1} N^{-1} \\
& \left|\left(\lambda_{2}\left(k_{3}^{\prime}-k_{1}^{\prime}\right)-\lambda_{2}\left(\tilde{k_{3}}-k_{1}^{\prime}\right)\right)-\left(\lambda_{3}\left(k_{3}^{\prime}\right)-\lambda_{3}\left(\tilde{k_{3}}\right)\right)\right| \lesssim A^{-1} N^{-1} .
\end{aligned}
$$

Define the vectors as

$\vec{v}_{1}=\lambda_{1}\left(k_{3}^{\prime}-k_{2}^{\prime}\right)-\lambda_{1}\left(\tilde{k_{3}}-k_{2}^{\prime}\right), \quad \vec{v}_{2}=\lambda_{2}\left(k_{3}^{\prime}-k_{1}^{\prime}\right)-\lambda_{2}\left(\tilde{k_{3}}-k_{1}^{\prime}\right), \quad \vec{v}_{3}=\lambda_{3}\left(k_{3}^{\prime}\right)-\lambda_{3}\left(\tilde{k_{3}}\right)$.

By the mean value theorem, there exist $\widehat{\lambda}_{i} \in S_{i}$ such that $\mathfrak{n}_{1}\left(\widehat{\lambda}_{i}\right) \perp \vec{v}_{i}$. This, (32), (33) and $\left|k_{3}^{\prime}-\tilde{k_{3}}\right| \gg 1$ which means $\left|\vec{v}_{3}\right| \gg N^{-1}$ provide

$$
\left|\operatorname{det}\left(\mathfrak{n}_{1}\left(\widehat{\lambda}_{1}\right), \mathfrak{n}_{2}\left(\widehat{\lambda}_{2}\right), \mathfrak{n}_{3}\left(\widehat{\lambda}_{3}\right)\right)\right| \ll A^{-1}
$$

which contradicts (13).

Next we consider the case $A^{-1} N^{-1} \ll L_{2} \leq N^{-1}$. By following the above argument, if $\left|k_{3}^{\prime}-\tilde{k_{3}}\right| \gg A L_{2}$ we can show $\left(S_{k_{3}^{\prime}, L_{2}}^{1} \times S_{k_{3}^{\prime}, L_{2}}^{2}\right) \cap\left(S_{\tilde{k_{3}}, L_{2}}^{1} \times S_{\tilde{k_{3}}, L_{2}}^{2}\right)=\emptyset$. Thus, after a harmless decomposition, it suffices to show that for any $k_{3}^{\prime} \in \operatorname{supp}_{k} f_{3}$ it holds

$$
\sum_{\left|k_{3}-k_{3}^{\prime}\right| \ll A L_{2}} \chi_{S_{k_{3}, L_{2}}^{1} \times S_{k_{3}, L_{2}}^{2}}\left(k_{1}, k_{2}\right) \lesssim\left\langle A N L_{2}\right\rangle .
$$

Without loss of generality, we may assume $k_{3}^{\prime}=(0,0)$ and $\left(k_{1}, k_{2}\right) \in S_{0, L_{2}}^{1} \times S_{0, L_{2}}^{2}$. Define

$$
K_{k_{1}, k_{2}, L_{2}}=\left\{k_{3} \in \mathbb{Z}^{2} / N|| k_{3} \mid \ll A L_{2},\left(k_{1}, k_{2}\right) \in S_{k_{3}, L_{2}}^{1} \times S_{k_{3}, L_{2}}^{2}\right\} .
$$

Our goal is to show $\# K_{k_{1}, k_{2}, L_{2}} \lesssim A N L_{2}$. Let $k_{3} \in K_{k_{1}, k_{2}, L_{2}}$. By following the same observation as in the former case $L_{2} \lesssim A^{-1} N^{-1}$, it follows from $0 \in K_{k_{1}, k_{2}, L_{2}}$, $k_{3} \in K_{k_{1}, k_{2}, L_{2}}$ that

$$
\begin{aligned}
& \left|\lambda_{1}\left(k_{1}\right)+\lambda_{2}\left(-k_{1}\right)-\lambda_{3}(0)\right| \lesssim L_{2}, \\
& \left|\lambda_{1}\left(-k_{2}\right)+\lambda_{2}\left(k_{2}\right)-\lambda_{3}(0)\right| \lesssim L_{2}, \\
& \left|\lambda_{1}\left(k_{1}\right)+\lambda_{2}\left(k_{3}-k_{2}\right)-\lambda_{3}\left(k_{3}\right)\right| \lesssim L_{2}, \\
& \left|\lambda_{1}\left(k_{3}-k_{2}\right)+\lambda_{2}\left(k_{2}\right)-\lambda_{3}\left(k_{3}\right)\right| \lesssim L_{2} .
\end{aligned}
$$

These yield

$$
\begin{aligned}
& \left|\left(\lambda_{1}\left(-k_{2}\right)-\lambda_{1}\left(k_{3}-k_{2}\right)\right)-\left(\lambda_{3}(0)-\lambda_{3}\left(k_{3}\right)\right)\right| \lesssim L_{2}, \\
& \left|\left(\lambda_{2}\left(-k_{1}\right)-\lambda_{2}\left(k_{3}-k_{1}\right)\right)-\left(\lambda_{3}(0)-\lambda_{3}\left(k_{3}\right)\right)\right| \lesssim L_{2} .
\end{aligned}
$$

Now we define the hypersurfaces $S_{1}^{\prime}$ and $S_{2}^{\prime}$ as

$$
S_{1}^{\prime}=S_{1}-\lambda_{1}\left(-k_{2}\right)+\lambda_{3}(0), \quad S_{2}^{\prime}=S_{2}-\lambda_{2}\left(-k_{1}\right)+\lambda_{3}(0) .
$$

Clearly, $\lambda_{3}(0) \in S_{1}^{\prime} \cap S_{2}^{\prime}$ and we deduce from (38) and (39) that

$$
\operatorname{dist}\left(\lambda_{3}\left(k_{3}\right), S_{1}^{\prime}\right) \lesssim L_{2}, \quad \operatorname{dist}\left(\lambda_{3}\left(k_{3}\right), S_{2}^{\prime}\right) \lesssim L_{2} .
$$


Consequently, $k_{3} \in K_{k_{1}, k_{2}, L_{2}}$ implies

$$
\begin{aligned}
k_{3} \in \tilde{K}_{k_{1}, k_{2}, L_{2}}:= & \left\{k_{3} \in \mathbb{Z}^{2} / N|| k_{3} \mid \ll A L_{2},\right. \\
& \left.\operatorname{dist}\left(\lambda_{3}\left(k_{3}\right), S_{1}^{\prime}\right)+\operatorname{dist}\left(\lambda_{3}\left(k_{3}\right), S_{2}^{\prime}\right) \lesssim L_{2}\right\},
\end{aligned}
$$

and it suffices to show $\# \tilde{K}_{k_{1}, k_{2}, L_{2}} \lesssim A N L_{2}$. To see this, we choose $\tilde{k}_{3} \in \tilde{K}_{k_{1}, k_{2}, L_{2}}$ which satisfies $\left|\tilde{k}_{3}\right| \sim \sup _{k_{3} \in \tilde{K}_{k_{1}, k_{2}, L_{2}}}\left|k_{3}\right|$. Clearly, $\left|\tilde{k}_{3}\right| \lesssim A^{1 / 2} N^{-1 / 2} L_{2}^{1 / 2}$ gives the desired estimate. Thus we assume $\left|\tilde{k}_{3}\right| \gg A^{1 / 2} N^{-1 / 2} L_{2}^{1 / 2}$. Further, for simplicity, we here assume that $\tilde{k}_{3}$ is on the first-axis, i.e. there exists $\tilde{k}_{3,1} \in \mathbb{Z} / N$ such that $\tilde{k}_{3}=\left(\tilde{k}_{3,1}, 0\right)$. For fixed $k_{3,1} \in \mathbb{Z} / N$ which satisfies $\left|k_{3,1}\right| \lesssim\left|\tilde{k}_{3,1}\right|$, we define

$$
\tilde{K}_{k_{1}, k_{2}, L_{2}}^{k_{3,1}}=\left\{k_{3,2} \in \mathbb{Z} / N \mid\left(k_{3,1}, k_{3,2}\right) \in \tilde{K}_{k_{1}, k_{2}, L_{2}}\right\}
$$

and show

$$
\# \tilde{K}_{k_{1}, k_{2}, L_{2}}^{k_{3,1}} \lesssim \max \left(A N L_{2}^{2} /\left|\tilde{k}_{3}\right|, 1\right),
$$

which gives the desired estimate as follows.

$$
\begin{aligned}
\# \tilde{K}_{k_{1}, k_{2}, L_{2}} & \sim\left|\tilde{k}_{3}\right| N \cdot \# \tilde{K}_{k_{1}, k_{2}, L_{2}}^{k_{3,1}} \\
& \lesssim \max \left(A L_{2}^{2} N^{2},\left|\tilde{k}_{3}\right| N\right) \lesssim A L_{2} N .
\end{aligned}
$$

Here we used $L_{2} \leq N^{-1}$ and $\left|\tilde{k}_{3}\right| \ll A L_{2}$. We prove (40) by contradiction. Assume that there exist $k_{3,2}, k_{3,2}^{\prime} \in \tilde{K}_{k_{1}, k_{2}, L_{2}}^{k_{3,1}}$ such that $d:=\left|k_{3,2}-k_{3,2}^{\prime}\right| \gg$ $\max \left(A L_{2}^{2} /\left|\tilde{k}_{3}\right|, 1 / N\right)$. We define $\sigma_{1}, \sigma_{2} \in \mathbb{S}^{2}$ as

$$
\sigma_{1}=\frac{\lambda(0)-\lambda\left(\tilde{k}_{3}\right)}{\left|\lambda(0)-\lambda\left(\tilde{k}_{3}\right)\right|}, \quad \sigma_{2}=\frac{\lambda\left(k_{3,1}, k_{3,2}\right)-\lambda\left(k_{3,1}, k_{3,2}^{\prime}\right)}{\left|\lambda\left(k_{3,1}, k_{3,2}\right)-\lambda\left(k_{3,1}, k_{3,2}^{\prime}\right)\right|} .
$$

Note that since $\left\|\nabla \psi_{3}\right\|_{L^{\infty}} \lesssim 1$ there exists a constant $0<c<1$ such that $\left|\sigma_{1} \cdot \sigma_{2}\right|<$ $1-c$. By the same observation as above, it follows from $0, \tilde{k}_{3} \in \tilde{K}_{k_{1}, k_{2}, L_{2}}$ that there exist $\lambda_{1}^{\prime} \in S_{1}^{\prime}, \lambda_{2}^{\prime} \in S_{2}^{\prime}, \lambda_{3}^{\prime} \in S_{3}$ such that $\left|\lambda_{i}^{\prime}-\lambda_{3}(0)\right| \lesssim\left|\tilde{k}_{3}\right|$ for $i=1,2,3$ and

$$
\left\{\mathfrak{n}_{1}^{\prime}\left(\lambda_{1}^{\prime}\right), \mathfrak{n}_{2}^{\prime}\left(\lambda_{2}^{\prime}\right), \mathfrak{n}_{3}\left(\lambda_{3}^{\prime}\right)\right\} \subset U_{\sigma_{1}}^{L_{2} /\left|\tilde{k}_{3}\right|}:=\left\{\sigma \in \mathbb{S}_{+}^{2}|| \sigma \cdot \sigma_{1}\left|\leq L_{2} /\right| \tilde{k}_{3} \mid\right\},
$$

where $\mathfrak{n}_{j}^{\prime}\left(\lambda_{j}\right)(j=1,2)$ is a unit normal on $\lambda_{j} \in S_{j}^{\prime}$ and $\mathbb{S}_{+}^{2}=\left\{(x, y, z) \in \mathbb{S}^{2} \mid z>0\right\}$. Similarly, $k_{3,2}, k_{3,2}^{\prime} \in \tilde{K}_{k_{1}, k_{2}, L_{2}}^{k_{3,1}}$ implies that there exist $\tilde{\lambda}_{1} \in S_{1}^{\prime}, \tilde{\lambda}_{2} \in S_{2}^{\prime}, \tilde{\lambda}_{3} \in S_{3}$ such that $\left|\tilde{\lambda}_{i}-\lambda_{3}(0)\right| \lesssim\left|\tilde{k}_{3}\right|$

$$
\left\{\mathfrak{n}_{1}^{\prime}\left(\tilde{\lambda}_{1}\right), \mathfrak{n}_{2}^{\prime}\left(\tilde{\lambda}_{2}\right), \mathfrak{n}_{3}\left(\tilde{\lambda}_{3}\right)\right\} \subset U_{\sigma_{2}}^{L_{2} / d}:=\left\{\sigma \in \mathbb{S}_{+}^{2}|| \sigma \cdot \sigma_{2} \mid \leq L_{2} / d\right\} .
$$

Our aim is to get

$\left|\mathfrak{n}_{1}^{\prime}\left(\lambda_{1}^{\prime}\right)-\mathfrak{n}_{2}^{\prime}\left(\lambda_{2}^{\prime}\right)\right|+\left|\mathfrak{n}_{2}^{\prime}\left(\lambda_{2}^{\prime}\right)-\mathfrak{n}_{3}\left(\lambda_{3}^{\prime}\right)\right|+\left|\mathfrak{n}_{3}\left(\lambda_{3}^{\prime}\right)-\mathfrak{n}_{1}^{\prime}\left(\lambda_{1}^{\prime}\right)\right| \lesssim \tilde{d}:=\left|\tilde{k}_{3}\right|+L_{2} / d+L_{2} /\left|\tilde{k}_{3}\right|$.

Note that $\tilde{d} \ll 1$. Since $\tilde{d} L_{2} /\left|\tilde{k}_{3}\right| \ll 1 / A$, we easily confirm that (41), (43) contradict the transversality condition (13). We turn to show (43). For the sake of contradiction, suppose that $\left|\mathfrak{n}_{1}^{\prime}\left(\lambda_{1}^{\prime}\right)-\mathfrak{n}_{2}^{\prime}\left(\lambda_{2}^{\prime}\right)\right| \gg \tilde{d}$. Firstly, we note that since $\left|\lambda_{i}^{\prime}-\lambda_{3}(0)\right|+\left|\tilde{\lambda}_{i}-\lambda_{3}(0)\right| \lesssim\left|\tilde{k}_{3}\right|$ and $S_{1}^{\prime}, S_{2}^{\prime}$ are $C^{1,1}$-hypersurfaces, we have

$$
\left|\mathfrak{n}_{1}^{\prime}\left(\lambda_{1}^{\prime}\right)-\mathfrak{n}_{1}^{\prime}\left(\tilde{\lambda}_{1}\right)\right|+\left|\mathfrak{n}_{2}^{\prime}\left(\lambda_{2}^{\prime}\right)-\mathfrak{n}_{2}^{\prime}\left(\tilde{\lambda}_{2}\right)\right| \lesssim\left|\tilde{k}_{3}\right| .
$$


We deduce from (41), (42), (44) and the assumption $\left|\mathfrak{n}_{1}^{\prime}\left(\lambda_{1}^{\prime}\right)-\mathfrak{n}_{2}^{\prime}\left(\lambda_{2}^{\prime}\right)\right| \gg \tilde{d}$ that there exist $s_{1}, s_{1}^{\prime}, s_{2}, s_{2}^{\prime} \in \mathbb{S}_{+}^{2}$ which satisy

$$
\begin{aligned}
& s_{1} \cdot \sigma_{1}=s_{1}^{\prime} \cdot \sigma_{2}=s_{2} \cdot \sigma_{1}=s_{2}^{\prime} \cdot \sigma_{2}=0, \\
& \left|s_{1}-s_{1}^{\prime}\right|+\left|s_{2}-s_{2}^{\prime}\right| \lesssim \tilde{d}, \quad\left|s_{1}-s_{2}\right| \gg \tilde{d},\left|s_{1}^{\prime}-s_{2}^{\prime}\right| \gg \tilde{d} .
\end{aligned}
$$

For $a, b \in \mathbb{R}^{3}, a \times b$ denotes the cross product of $a$ and $b$. We see that the above contradicts $\left|\sigma_{1} \cdot \sigma_{2}\right|<1-c$ as follows.

$$
\begin{aligned}
\left|\sigma_{1} \cdot \sigma_{2}\right| & =\frac{\left|\left(s_{1} \times s_{2}\right) \cdot\left(s_{1}^{\prime} \times s_{2}^{\prime}\right)\right|}{\left|s_{1} \times s_{2}\right|\left|s_{1}^{\prime} \times s_{2}^{\prime}\right|} \\
& \geq \frac{\left|s_{1} \times s_{2}\right|-\left|s_{1}-s_{1}^{\prime}\right|-\left|s_{2}-s_{2}^{\prime}\right|}{\left|s_{1} \times s_{2}\right|+\left|s_{1}-s_{1}^{\prime}\right|+\left|s_{2}-s_{2}^{\prime}\right|} \\
& >1-c / 2 .
\end{aligned}
$$

Here we used $\left|s_{1} \times s_{2}\right| \gg \tilde{d}$ which follows from $\left|s_{1}-s_{2}\right| \gg \tilde{d}$ and $\tilde{d} \ll 1$.

By using the estimate (26), we complete the proof of (24) as the proof of Theorem 4.3 .

4.4. Examples. At last, we consider an example to compare Loomis-Whitney inequalities in $\mathbb{R}^{3}$ to the $\mathbb{R} \times$ lattice case.

Let $\psi(\xi, \eta)=\xi^{3}+\eta^{3}$ and consider the surface

$$
S=\left\{(\psi(\xi, \eta), \xi, \eta) \mid(\xi, \eta) \in \mathbb{R}^{2}\right\} .
$$

Let $\left(\mathcal{U}_{i}\right)_{i=1}^{3}$ denote neighborhoods of $(N,-N),(N, 2 N)$ and $(2 N, N)$. Let $f_{i} \in$ $L^{2}\left(\mathbb{R}^{3}\right), f_{i} \geq 0$ and suppose that $\operatorname{supp}\left(f_{i}\right) \subseteq\left\{(\xi, \eta, \tau) \in \mathbb{R}^{3}\left|(\xi, \eta) \in \mathcal{U}_{i},\right| \tau-\right.$ $\left.\psi(\xi, \eta) \mid \leq L_{i}\right\}$. After rescaling, $(\xi, \eta) \rightarrow(\xi, \eta) / N, \tau \rightarrow \tau / N^{3}$ we find $\tilde{f}_{i} \in L^{2}\left(\mathbb{R}^{3}\right)$ supported in a fixed compact set. Moreover, we have

$$
\left|\operatorname{det}\left(\mathfrak{n}\left(\xi_{1}, \eta_{1}\right), \mathfrak{n}\left(\xi_{2}, \eta_{2}\right), \mathfrak{n}\left(\xi_{1}+\xi_{2}, \eta_{1}+\eta_{2}\right)\right)\right| \gtrsim 1 .
$$

An application of Fubini's theorem and [3, Corollary 1.6., p. 713]

$$
\int_{\mathbb{R}^{3}}\left(f_{1} * f_{2}\right)(\tau, \xi, \eta) f_{3}(\tau, \xi, \eta) d \xi d \eta d \tau \lesssim N^{-2}\left(L_{1} L_{2} L_{3}\right)^{1 / 2} \prod_{i=1}^{3}\left\|f_{i}\right\|_{L^{2}} .
$$

In the periodic case we consider $f_{i}: \mathbb{R} \times \mathbb{Z}^{2} \rightarrow \mathbb{R}$. It is easy to see choosing $f_{1}$ with $\mathbb{Z}^{2}$-support $(N,-N), f_{2}$ with $\mathbb{Z}^{2}$-support $(N, 2 N)$ and $f_{3}$ with $\mathbb{Z}^{2}$-support $(2 N, N)$ that

$$
\int_{\mathbb{R} \times \mathbb{Z}^{2}}\left(f_{1} * f_{2}\right)(\tau, \xi, \eta) f_{3}(\tau, \xi, \eta)(d \xi)_{1}(d \eta)_{1} d \tau \sim L_{\min }^{1 / 2} \prod_{i=1}^{3}\left\|f_{i}\right\|_{L^{2}\left(\mathbb{R} \times \mathbb{Z}^{2}\right)} .
$$

\section{Energy estimates}

The main result of this section is the following proposition:

Proposition 5.1. Let $\lambda \geq 1, T \in(0,1], 1<s \leq s^{\prime}$ and $u_{1}, u_{2} \in C\left([0, T], H_{\lambda}^{3}\right)$ be $\lambda$-periodic classical solutions to (1). Set $v=u_{1}-u_{2}$. Then, we find the following 
estimates to hold:

$$
\begin{aligned}
\left\|u_{1}\right\|_{E_{\lambda}^{s^{\prime}}(T)}^{2} & \lesssim\left\|u_{1}(0)\right\|_{H_{\lambda}^{s^{\prime}}}^{2}+\left\|u_{1}\right\|_{F_{\lambda}^{s^{\prime}}(T)}^{2}\left\|u_{1}\right\|_{F_{\lambda}^{s}(T)}, \\
\|v\|_{E_{\lambda}^{0}(T)}^{2} & \lesssim\|v(0)\|_{L_{\lambda}^{2}}^{2}+\|v\|_{F_{\lambda}^{0}(T)}^{2}\left(\left\|u_{1}\right\|_{F_{\lambda}^{s}(T)}+\left\|u_{2}\right\|_{F_{\lambda}^{s}(T)}\right), \\
\|v\|_{E_{\lambda}^{s^{\prime}}(T)}^{2} & \lesssim\|v(0)\|_{H_{\lambda}^{s^{\prime}}}^{2}+\|v\|_{F_{\lambda}^{0}(T)}\|v\|_{F_{\lambda}^{s^{\prime}(T)}}\left\|u_{2}\right\|_{F_{\lambda}^{2 s^{\prime}(T)}}+\|v\|_{F_{\lambda}^{s^{\prime}(T)}}^{2}\|v\|_{F_{\lambda}^{s}(T)} .
\end{aligned}
$$

At the end of the section, we provide an example indicating that the methods of this paper give estimates that are sharp up to endpoints in terms of Sobolev regularity.

For the proof of Proposition 5.1, we write by the fundamental theorem of calculus for a solution $u \in C\left([0, T], H_{\lambda}^{3}\right)$ to (1) on $\lambda \mathbb{T}^{2}$ :

$$
\left\|P_{N} u(t)\right\|_{L^{2}\left(\lambda \mathbb{T}^{2}\right)}^{2}=\left\|P_{N} u(0)\right\|_{L_{\lambda}^{2}}^{2}+2 \int_{0}^{t} \int_{\lambda \mathbb{T}^{2}} P_{N} u(s, x) \partial_{x} P_{N}\left(u^{2}\right)(s, x) d x d s .
$$

To exploit the form of the nonlinearity, we integrate by parts to put the derivative on the lowest frequency. We sketch the necessary standard arguments, for details we refer to previous works $[15,28]$ :

For $K \ll N$,

$$
\begin{aligned}
& \int_{0}^{T} \int_{\lambda \mathbb{T}^{2}} P_{N} u(s, x) \partial_{x} P_{N}\left(u P_{K} u\right)(s, x) d x d s \\
= & \int_{0}^{T} \int_{\lambda \mathbb{T}^{2}} P_{N} u(s, x) \partial_{x}\left[\left(P_{N} u P_{K} u\right)+\left[P_{N}\left(u P_{K} u\right)-P_{N} u P_{K} u\right]\right](s, x) d x d s \\
= & \frac{1}{2} \int_{0}^{T} \int_{\lambda \mathbb{T}^{2}}\left(P_{N} u\right)^{2}(s, x)\left(\partial_{x} P_{K} u\right)(s, x) d x d s \\
& +\int_{0}^{T} \int_{\lambda \mathbb{T}^{2}}\left(P_{N} u\right)(s, x) \partial_{x}\left[P_{N}\left(u P_{K} u\right)-P_{N} u P_{K} u\right](s, x) d x d s=A+B .
\end{aligned}
$$

$A$ is already in suitable form. For $B$, we change to Fourier variables to write by the mean value theorem

$$
\begin{aligned}
& \int_{\lambda \mathbb{T}^{2}}\left(P_{N} u\right)(s, x) \partial_{x}\left[P_{N}\left(u P_{K} u\right)-P_{N} u P_{K} u\right](s, x) d x \\
= & \frac{1}{\lambda^{4}} \sum_{\substack{k_{1}+k_{2}+k_{3}=0, k_{i} \in \mathbb{Z}^{2} / \lambda}} \chi_{N}\left(k_{1}\right)\left(-i k_{1,1}\right)\left[\chi_{N}\left(k_{2}+k_{3}\right)-\chi_{N}\left(k_{2}\right)\right] \chi_{K}\left(k_{3}\right) \prod_{i=1}^{3} \hat{u}\left(s, k_{i}\right) \\
= & \frac{1}{\lambda^{4}} \sum_{\substack{k_{1}+k_{2}+k_{3}=0, k_{i} \in \mathbb{Z}^{2} / \lambda \\
\text { where }|\zeta| \sim N .}} \chi_{N}\left(k_{1}\right) \hat{u}\left(k_{1}\right)\left(-i k_{1,1}\right)\left(\nabla \chi_{N}(\zeta) \cdot k_{3}\right) \hat{u}\left(k_{2}\right) \chi_{K}\left(k_{3}\right) \hat{u}\left(s, k_{3}\right),
\end{aligned}
$$

In the following let $\lambda \geq 1$ denote the period length, $\xi, \eta \in \mathbb{R}$, and we denote $\left(d \sigma_{i}\right)_{\lambda}=d \tau_{i}\left(d k_{i}\right)_{\lambda}$ and

$$
\begin{aligned}
& \int_{*} f_{1}\left(\tau_{1}, k_{1}\right) f_{2}\left(\tau_{2}, k_{2}\right) f_{3}\left(\tau_{3}, k_{3}\right)\left(d \sigma_{1}\right)_{\lambda}\left(d \sigma_{2}\right)_{\lambda} \\
= & \int_{\left(\mathbb{R} \times \mathbb{Z}^{2} / \lambda\right)^{2}} f_{1}\left(\tau_{1}, k_{1}\right) f_{2}\left(\tau_{2}, k_{2}\right) f_{3}\left(\tau_{1}+\tau_{2}, k_{1}+k_{2}\right)\left(d \sigma_{1}\right)_{\lambda}\left(d \sigma_{2}\right)_{\lambda} .
\end{aligned}
$$


To estimate the frequency localized functions in the short-time function spaces $F_{n, \lambda}$, time has to be localized reciprocally to the highest occuring frequency. The reductions are standard and can already be found in [15, Section 5]. Taking absolute values, we find that the estimates from Proposition 5.1 are implied by the following:

Proposition 5.2. Let $\lambda \geq 1$. Assume that $1 \ll N_{3} \lesssim N_{2} \leq N_{1}, L_{\text {med }} \leq N_{1}^{2}$, $f_{i}: \mathbb{R} \times \mathbb{Z}^{2} / \lambda \rightarrow \mathbb{R}_{\geq 0}$ and $\operatorname{supp} f_{i} \subset G_{N_{i}, L_{i}}$. Then, we have

$$
\begin{aligned}
& \left|\int_{*}\left(\left|k_{3,1}\right|+\left|k_{1,1}\right| \frac{N_{3}}{N_{1}}\right) f_{1}\left(\tau_{1}, k_{1}\right) f_{2}\left(\tau_{2}, k_{2}\right) f_{3}\left(\tau_{3}, k_{3}\right)\left(d \sigma_{1}\right)_{\lambda}\left(d \sigma_{2}\right)_{\lambda}\right| \\
\lesssim & N_{3}^{1+\varepsilon} L_{\min }^{\frac{1}{2}}\left\langle N_{1}^{-\frac{1}{2}} L_{\max }^{\frac{1}{2}}\right\rangle\left\|f_{1}\right\|_{L_{\tau}^{2} L_{(d k)_{\lambda}}^{2}}\left\|f_{2}\right\|_{L_{\tau}^{2} L_{(d k)_{\lambda}}^{2}}\left\|f_{3}\right\|_{L_{\tau}^{2} L_{(d k)_{\lambda}}^{2}} .
\end{aligned}
$$

Note that Proposition 5.1 in the case of large modulations $L_{\text {med }} \gtrsim N_{1}^{2}$ follows from the Cauchy-Schwarz inequality (cf. Lemma 5.7). For the same reason, we can suppose that $N_{3} \gg 1$.

We record estimates, which will be used in the proof. Set $\psi(\xi, \eta)=\xi\left(\xi^{2}+\eta^{2}\right)$.

Proposition 5.3. Let $K_{1}, K_{2}, K_{3} \subset \mathbb{R}^{2}$ satisfy for $i=1,2,3$

$$
\sup _{\left(\xi_{i}, \eta_{i}\right),\left(\xi_{i}^{\prime}, \eta_{i}^{\prime}\right) \in K_{i}}\left|\nabla \psi\left(\xi_{i}, \eta_{i}\right)-\nabla \psi\left(\xi_{i}^{\prime}, \eta_{i}^{\prime}\right)\right| \ll A^{-1} N_{1}^{2},
$$

and for all $\left(\xi_{1}, \eta_{1}\right) \in K_{1},\left(\xi_{2}, \eta_{2}\right) \in K_{2}$

$$
\left|\left(\xi_{1} \eta_{2}-\xi_{2} \eta_{1}\right)\left(3\left(\xi_{1}^{2}+\xi_{1} \xi_{2}+\xi_{2}^{2}\right)-\left(\eta_{1}^{2}+\eta_{1} \eta_{2}+\eta_{2}^{2}\right)\right)\right| \gtrsim A^{-1} N_{1}^{4},
$$

and $\tilde{K}_{i}=\mathbb{R} \times K_{i}$. Assume that $1 \leq A \leq N_{1}, 1 \leq N_{3} \lesssim N_{1} \sim N_{2}$ and $f_{i}(i=1,2,3)$ satisfy supp $f_{i} \subset G_{N_{i}, L_{i}}$. Then, we find the following estimate to hold:

$$
\begin{aligned}
& \left.\left.\left|\int_{*} f_{1}\right|_{\tilde{K}_{1}}\left(\tau_{1}, k_{1}\right) f_{2}\right|_{\tilde{K}_{2}}\left(\tau_{2}, k_{2}\right) f_{3}\right|_{\tilde{K}_{3}}\left(\tau_{3}, k_{3}\right)\left(d \sigma_{1}\right)_{\lambda}\left(d \sigma_{2}\right)_{\lambda} \mid \\
\lesssim & \tilde{C}\left(A, N, L_{1}, L_{2}, L_{3}\right)\left\|f_{1}\right\|_{L_{\tau}^{2} L_{(d k)}^{2}}\left\|f_{2}\right\|_{L_{\tau}^{2} L_{(d k)}^{2}}\left\|f_{3}\right\|_{L_{\tau}^{2} L_{(d k)_{\lambda}}^{2}},
\end{aligned}
$$

where

$$
\tilde{C}\left(A, N_{1}, L_{1}, L_{2}, L_{3}\right)=L_{\min }^{1 / 2}\left\langle L_{\text {med }} N_{1}^{-2}\right\rangle^{1 / 2}\left\langle A L_{\max } N_{1}^{-2}\right\rangle^{1 / 2} .
$$

Proof. We note that

$$
\tilde{C}\left(A, N_{1}, L_{1}, L_{2}, L_{3}\right)=\left\{\begin{array}{ll}
L_{\min }^{\frac{1}{2}}\left\langle A N_{1}^{-2} L_{\text {max }}\right\rangle^{\frac{1}{2}}, & L_{\text {med }} \leq N_{1}^{2}, \\
\left(A L_{1} L_{2} L_{3}\right)^{\frac{1}{2}} N_{1}^{-2}, & N_{1}^{2} \leq L_{\text {med }}
\end{array} .\right.
$$

If we define $\tilde{f}_{i}(\tau, k)=f_{i}\left(N_{1}^{3} \tau, N_{1} k\right)$, these satisfy $\operatorname{supp} \tilde{f}_{i} \subset G_{N_{i} / N_{1}, L_{i} / N_{1}^{3}}$, and the claim can be rewritten as follows:

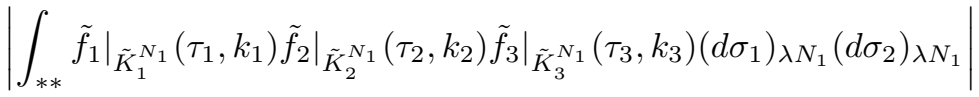

$$
\begin{aligned}
& \lesssim N_{1}^{-\frac{5}{2}} \tilde{C}\left(A, N, L_{1}, L_{2}, L_{3}\right)\left\|\tilde{f}_{1}\right\|_{L_{\tau}^{2} L_{(d k)}^{2} \lambda_{N_{1}}}\left\|\tilde{f}_{2}\right\|_{L_{\tau}^{2} L_{(d k)}^{2} \lambda N_{1}}\left\|\tilde{f}_{3}\right\|_{L_{\tau}^{2} L_{(d k)}^{2} \lambda_{1}},
\end{aligned}
$$

where $* *=\left(\mathbb{R} \times \mathbb{Z}^{2} / \lambda N_{1}\right)^{2}, K_{i}^{N_{1}}=\left\{(\xi, \eta) \in \mathbb{R}^{2} \mid\left(N_{1} \xi, N_{1} \eta\right) \in K_{i}\right\}$ and $\tilde{K}_{i}^{N_{1}}=$ $\mathbb{R} \times K_{i}^{N_{1}}$. Define $\tilde{L}_{i}=N_{1}^{-3} L_{i}$. Then, by using the notation $C\left(A, N, L_{1}, L_{2}, L_{3}\right)$ defined in (25), (50) is implied by

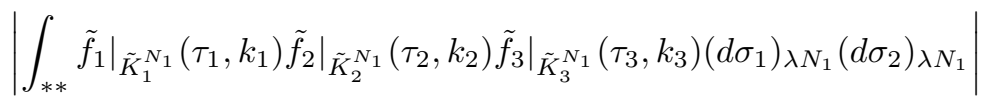

$$
\begin{aligned}
& \lesssim C\left(A, \lambda N_{1}, \tilde{L}_{1}, \tilde{L}_{2}, \tilde{L}_{3}\right) /\left(\lambda N_{1}\right)\left\|\tilde{f}_{1}\right\|_{L_{\tau}^{2} L_{(d k)}^{2} \lambda N_{1}}\left\|\tilde{f}_{2}\right\|_{L_{\tau}^{2} L_{(d k)_{\lambda N_{1}}}^{2}}\left\|\tilde{f}_{3}\right\|_{L_{\tau}^{2} L_{(d k)}^{2} N_{1}} .
\end{aligned}
$$


We define

$$
S_{i}=\left\{(\psi(\xi, \eta), \xi, \eta) \in \mathbb{R}^{3}\left|(\xi, \eta) \in K_{i}^{N_{1}},\right|(\xi, \eta) \mid \lesssim 1\right\} .
$$

(51) is immediately established by Proposition 4.8 if the hypersurfaces $S_{1}, S_{2}, S_{3}$ satisfy Assumption 1. Since $\psi$ is a polynomial function, we only need to confirm that the hypersurfaces satisfy the necessary transversality condition. To show this, we describe the unit normals $\mathfrak{n}_{i}$ on $\lambda_{i}=\left(\psi\left(\xi_{i}, \eta_{i}\right), \xi_{i}, \eta_{i}\right) \in S_{i}$ explicitly:

$$
\mathfrak{n}_{i}\left(\lambda_{i}\right)=\frac{1}{\sqrt{1+\left(3 \xi_{i}^{2}+\eta_{i}^{2}\right)^{2}+4 \xi_{i}^{2} \eta_{i}^{2}}}\left(-1,3 \xi_{i}^{2}+\eta_{i}^{2}, 2 \xi_{i} \eta_{i}\right)
$$

We can assume that there exist $\widehat{\lambda}_{i}=\left(\psi\left(\widehat{\xi}_{i}, \widehat{\eta}_{i}\right),\left(\widehat{\xi}_{i}, \widehat{\eta}_{i}\right)\right) \in S_{i}$ such that $\widehat{\lambda}_{1}+\widehat{\lambda}_{2}=\widehat{\lambda}_{3}$. It is easily observed that (48) provides

$$
\sup _{\lambda_{i}, \lambda_{i}^{\prime} \in S_{i}}\left|\mathfrak{n}_{i}\left(\lambda_{i}\right)-\mathfrak{n}_{i}\left(\lambda_{i}^{\prime}\right)\right| \ll A^{-1} .
$$

Therefore, it suffices to show

$$
\left|\operatorname{det}\left(\mathfrak{n}_{1}\left(\widehat{\lambda}_{1}\right), \mathfrak{n}_{2}\left(\widehat{\lambda}_{2}\right), \mathfrak{n}_{3}\left(\widehat{\lambda}_{3}\right)\right)\right| \gtrsim A^{-1}
$$

which follows from the condition (49) as follows:

$$
\begin{aligned}
& \left|\operatorname{det}\left(\mathfrak{n}_{1}\left(\widehat{\lambda}_{1}\right), \mathfrak{n}_{2}\left(\widehat{\lambda}_{2}\right), \mathfrak{n}_{3}\left(\widehat{\lambda}_{3}\right)\right)\right| \\
\gtrsim & \left|\operatorname{det}\left(\begin{array}{ccc}
-1 & -1 & -1 \\
3 \widehat{\xi}_{1}^{2}+\widehat{\eta}_{1}^{2} & 3 \widehat{\xi}_{2}^{2}+\widehat{\eta}_{2}^{2} & 3 \widehat{\xi}_{3}^{2}+\widehat{\eta}_{3}^{2} \\
2 \widehat{\xi}_{1} \widehat{\eta}_{1} & 2 \widehat{\xi}_{2} \widehat{\eta}_{2} & 2 \widehat{\xi}_{3} \widehat{\eta}_{3}
\end{array}\right)\right| \\
\gtrsim & \left|\left(\widehat{\xi}_{1} \widehat{\eta}_{2}-\widehat{\xi}_{2} \widehat{\eta}_{1}\right)\left(3\left(\widehat{\xi}_{1}^{2}+\widehat{\xi}_{1} \widehat{\xi}_{2}+\widehat{\xi}_{2}^{2}\right)-\left(\widehat{\eta}_{1}^{2}+\widehat{\eta}_{1} \widehat{\eta}_{2}+\widehat{\eta}_{2}^{2}\right)\right)\right| \\
\gtrsim & A^{-1} .
\end{aligned}
$$

It is known that in $\mathbb{R}^{2}$ a linear transformation (cf. [4, 13]) allows for a symmetrization of the Zakharov-Kuznetsov equation to the following (up to irrelevant factors)

$$
\partial_{t} u+\left(\partial_{x_{1}}^{3}+\partial_{x_{2}}^{3}\right) u=u\left(\partial_{x_{1}}+\partial_{x_{2}}\right) u
$$

We digress for a moment to consider the effect of this transformation:

$$
\partial_{t} u+\partial_{x_{1}}^{3} u+\partial_{x_{1}} \partial_{x_{2}}^{2} u=u \partial_{x_{1}} u, \quad(t, x) \in \mathbb{R} \times \lambda \mathbb{T}^{2}, \quad \lambda>0 .
$$

In Fourier space we can morally still consider the symmetrized equation (52). However, the Fourier variables $(\xi, \eta)$ have to satisfy the following equation:

$$
\begin{aligned}
\xi & =\sqrt{2}\left(\alpha+3^{-1 / 2} \beta\right) \\
\eta & =\sqrt{2}\left(\alpha-3^{-1 / 2} \beta\right)
\end{aligned}
$$

for $(\alpha, \beta) \in \mathbb{Z}^{2} / \lambda$. When we wants to use the orthogonal decompositions from [17], we can do so after taking into account that the Fourier support of the "symmetrized" equation (52) is on $M\left(\mathbb{Z}^{2} / \lambda\right)$, where

$$
M=\sqrt{2}\left(\begin{array}{cc}
1 & 3^{-1 / 2} \\
1 & -3^{-1 / 2}
\end{array}\right), \quad M^{-1}=2^{-3 / 2}\left(\begin{array}{cc}
1 & 1 \\
3^{1 / 2} & -3^{1 / 2}
\end{array}\right) .
$$

If we want to compute the measure of a set $S$ with respect to counting measure on $M\left(\mathbb{Z}^{2} / \lambda\right)$ it is more convenient to apply $M^{-1}$ and count the lattice points of $\mathbb{Z}^{2} / \lambda$ in $S^{\prime}:=M^{-1} S$. 
For one of the critical interactions, we have to estimate the number of points of $M\left(\mathbb{Z}^{2} / \lambda\right)$ in a rectangle parallel to the $\eta$-axis with height $N_{2}$ and width $\ll N_{1}^{-1}$ with $N_{2} \ll N_{1}$. See Lemma 5.9 .

A lattice point $(q, p) \in \mathbb{N} \times \mathbb{Z}$ is in the rotated rectangle parallel to the line $\eta=\sqrt{3} \xi$ with width $\ll N_{1}^{-1}$ if and only if $(q, p)$ satisfies

$$
|\sqrt{3} q-p| \ll \frac{1}{N_{1}} \Leftrightarrow\left|\sqrt{3}-\frac{p}{q}\right| \ll \frac{1}{N_{1} q} .
$$

Here we invoke Liouville's theorem on diophantine approximation:

Theorem 5.4. If $x$ is an irrational algebraic number of degree $n$ over the rational numbers, then there exists a constant $c(x)>0$ such that

$$
\left|x-\frac{p}{q}\right|>\frac{c(x)}{q^{n}}
$$

holds for all integers $p$ and $q$ where $q>0$.

Hence, since $\sqrt{3}$ is an irrational algebraic number of degree 2 , for $0<q \ll N_{1}$, the inequality (56) has no solution since

$$
\left|\sqrt{3}-\frac{p}{q}\right| \leq \frac{1}{N_{1} q} \leq \frac{c(\sqrt{3})}{q^{2}} .
$$

The following lemma will be needed:

Lemma 5.5. Let $\lambda \geq 1, \ell, w>0$ such that $\ell w \geq 1$ and $\alpha \in \mathbb{R}^{2}$. Define the vectors $\overrightarrow{v_{1}}=(1, \sqrt{3}), \overrightarrow{v_{2}}=(-1, \sqrt{3})$ and

$$
\begin{aligned}
& S_{\ell, w}^{\alpha}=\left\{(\xi, \eta) \in \mathbb{R}^{2}\left|(\xi, \eta)=c_{1} \vec{v}_{1}+c_{2} \vec{v}_{2}, \quad\right| c_{1}|\leq \ell,| c_{2} \mid \leq w\right\}-\alpha, \\
& \tilde{S}_{\ell, w}^{\alpha}=\left\{k \in \mathbb{Z}^{2} / \lambda \cap S_{\ell, w}^{\alpha}\right\} .
\end{aligned}
$$

Then, we have $\sup _{\alpha \in \mathbb{R}^{2}} \# \tilde{S}_{\ell, w}^{\alpha} \lesssim \ell w \lambda^{2}$.

Remark 5.6. Observe how the argument hinges on the ratio of the period lengths. We can still apply Theorem 5.4 if the ratio of the period lengths is rational. On the other hand, if $k \in \mathbb{Z} / \lambda \times \sqrt{3} \mathbb{Z} / \lambda$ this lemma does not hold true. Indeed, for $\ell \gg 1$ and $0<w \ll 1$ we find that $\#\left\{k \in \mathbb{Z} / \lambda \times \sqrt{3} \mathbb{Z} / \lambda \cap S_{\ell, w}^{0}\right\} \sim \ell \lambda^{2}$.

Proof. We can assume $\lambda=1$ by rescaling. By performing a suitable decomposition, it suffices to show $\sup _{\alpha \in \mathbb{R}^{2}} \# \tilde{S}_{\ell, w}^{\alpha} \lesssim 1$ for $w \ll \ell^{-1}$. Assume that $\tilde{S}_{\ell, w}^{\alpha}$ is not empty. Then, after parallel translation, it suffices to show $\# \tilde{S}_{2 \ell, 2 w}^{0}=1$ which is verified by Theorem 5.4 as above observation.

The following estimate follows from the Cauchy-Schwarz inequality.

Lemma 5.7. For $i=1,2,3$, assume that $f_{i}: \mathbb{R} \times \mathbb{Z}^{2} / \lambda \rightarrow \mathbb{R}_{\geq 0}$, supp $f_{i} \subset G_{N_{i}, L_{i}}$ and $\min _{i=1,2,3} \# \operatorname{supp}_{k} f_{i} \lesssim P$. Then we have

$$
\begin{aligned}
& \left|\int_{*} f_{1}\left(\tau_{1}, k_{1}\right) f_{2}\left(\tau_{2}, k_{2}\right) f_{3}\left(\tau_{3}, k_{3}\right)\left(d \sigma_{1}\right)_{\lambda}\left(d \sigma_{2}\right)_{\lambda}\right| \\
\lesssim & \left(P L_{\min }\right)^{\frac{1}{2}} / \lambda\left\|f_{1}\right\|_{L_{\tau}^{2} L_{(d k)}^{2}}\left\|f_{2}\right\|_{L_{\tau}^{2} L_{(d k)_{\lambda}}^{2}}\left\|f_{3}\right\|_{L_{\tau}^{2} L_{(d k)_{\lambda}}^{2}} .
\end{aligned}
$$


We begin the proof in earnest, for which we consider the two cases:

(I) $\max \left(\left|k_{1,1}\right|,\left|k_{2,1}\right|\right) \geq 2^{-5} N_{1}, \quad$ (II) $\max \left(\left|k_{1,1}\right|,\left|k_{2,1}\right|\right) \leq 2^{-5} N_{1}$.

First we consider the case (I). Since $\left|k_{3,1}\right|+\left|k_{1,1}\right| N_{3} / N_{1} \lesssim N_{3}$, it suffices to show

$$
\begin{aligned}
& \left|\int_{*} f_{1}\left(\tau_{1}, k_{1}\right) f_{2}\left(\tau_{2}, k_{2}\right) f_{3}\left(\tau_{3}, k_{3}\right)\left(d \sigma_{1}\right)_{\lambda}\left(d \sigma_{2}\right)_{\lambda}\right| \\
\lesssim & N_{3}^{\varepsilon} L_{\min }^{\frac{1}{2}}\left\langle N_{1}^{-\frac{1}{2}} L_{\max }^{\frac{1}{2}}\right\rangle\left\|f_{1}\right\|_{L_{\tau}^{2} L_{(d k)_{\lambda}}^{2}}\left\|f_{2}\right\|_{L_{\tau}^{2} L_{(d k)_{\lambda}}^{2}}\left\|f_{3}\right\|_{L_{\tau}^{2} L_{(d k)_{\lambda}}^{2}} .
\end{aligned}
$$

We perform the linear transformation

$$
(x, y) \rightarrow M(x, y)=\sqrt{2}(x+y / \sqrt{3}, x-y / \sqrt{3})
$$

and show the following estimate which is equivalent to (57):

$$
\begin{aligned}
& \left|\int_{*} g_{1}\left(\tau_{1}, \ell_{1}\right) g_{2}\left(\tau_{2}, \ell_{2}\right) g_{3}\left(\tau_{3}, \ell_{3}\right)\left(d \tilde{\sigma}_{1}\right)_{\lambda}\left(d \tilde{\sigma}_{2}\right)_{\lambda}\right| \\
\lesssim & N_{3}^{\varepsilon} L_{\min }^{\frac{1}{2}}\left\langle N_{1}^{-\frac{1}{2}} L_{\max }^{\frac{1}{2}}\right\rangle\left\|g_{1}\right\|_{L_{\tau}^{2} L_{(d \ell)_{\lambda}}^{2}}\left\|g_{2}\right\|_{L_{\tau}^{2} L_{(d \ell)_{\lambda}}^{2}}\left\|g_{3}\right\|_{L_{\tau}^{2} L_{(d \ell)_{\lambda}}^{2}},
\end{aligned}
$$

where, for $\ell \in \mathbb{R}^{2}$ letting $\tilde{\psi}(\ell)=\tilde{\psi}\left(\ell_{1}, \ell_{2}\right)=\ell_{1}^{3}+\ell_{2}^{3}$,

(59) $\operatorname{supp} g_{i} \subset \tilde{G}_{N_{i}, L_{i}}, \quad \tilde{G}_{N, L}=\left\{(\tau, \ell) \in \mathbb{R} \times M\left(\mathbb{Z}^{2} / \lambda\right)|| \ell|\sim N,| \tau-\tilde{\psi}(\ell) \mid \lesssim L\right\}$.

In $(58)\left(d \tilde{\sigma}_{i}\right)_{\lambda}$ denotes the image measure under the linear transformation of $\left(d \sigma_{i}\right)_{\lambda}$; similarly, for $(d \ell)_{\lambda}$ and $(d k)_{\lambda}$.

As above, the advantage of considering (58) over (57) is that we can reuse the Whitney type decompositions from [17].

We note that the assumption (I) $\max \left(\left|k_{1,1}\right|,\left|k_{2,1}\right|\right) \geq 2^{-5} N_{1}$ provides $\max \left(\mid \ell_{1,1}+\right.$ $\left.\ell_{1,2}|,| \ell_{2,1}+\ell_{2,2} \mid\right) \geq 2^{-6} N_{1}$ in (58). For convenience, performing the linear transformation $M$, we state the estimates that correspond to Proposition 5.3 and Lemmas 5.5 and 5.7 .

Proposition 5.8. Let $K_{1}, K_{2}, K_{3} \subset \mathbb{R}^{2}$ satisfy for $i=1,2,3$

$$
\sup _{\left(\xi_{i}, \eta_{i}\right),\left(\xi_{i}^{\prime}, \eta_{i}^{\prime}\right) \in K_{i}}\left|\nabla \tilde{\psi}\left(\xi_{i}, \eta_{i}\right)-\nabla \tilde{\psi}\left(\xi_{i}^{\prime}, \eta_{i}^{\prime}\right)\right| \ll A^{-1} N_{1}^{2},
$$

and for all $\left(\xi_{1}, \eta_{1}\right) \in K_{1},\left(\xi_{2}, \eta_{2}\right) \in K_{2}$

$$
\left|\left(\xi_{1} \eta_{2}-\xi_{2} \eta_{1}\right)\left(\xi_{1} \eta_{2}+\xi_{2} \eta_{1}+2\left(\xi_{1} \eta_{1}+\xi_{2} \eta_{2}\right)\right)\right| \gtrsim A^{-1} N_{1}^{4}
$$

and $\tilde{K}_{i}=\mathbb{R} \times K_{i}$. Assume that $1 \leq A \leq N_{1}, 1 \leq N_{3} \lesssim N_{1} \sim N_{2}$ and $g_{i}(i=1,2,3)$ satisfy (59). Then, we have

$$
\begin{aligned}
& \left.\left.\left|\int_{*} g_{1}\right|_{\tilde{K}_{1}}\left(\tau_{1}, \ell_{1}\right) g_{2}\right|_{\tilde{K}_{2}}\left(\tau_{2}, \ell_{2}\right) g_{3}\right|_{\tilde{K}_{3}}\left(\tau_{3}, \ell_{3}\right)\left(d \tilde{\sigma}_{1}\right)_{\lambda}\left(d \tilde{\sigma}_{2}\right)_{\lambda} \mid \\
\lesssim & \tilde{C}\left(A, N, L_{1}, L_{2}, L_{3}\right)\left\|g_{1}\right\|_{L_{\tau}^{2} L_{(d \ell)_{\lambda}}^{2}}\left\|g_{2}\right\|_{L_{\tau}^{2} L_{(d \ell)_{\lambda}}^{2}}\left\|g_{3}\right\|_{L_{\tau}^{2} L_{(d \ell)_{\lambda}}^{2}} .
\end{aligned}
$$

Since $M \overrightarrow{v_{1}}=(\sqrt{2}, 0), M \overrightarrow{v_{2}}=(0,-\sqrt{2})$, we find that Lemma 5.5 is equivalent to the following:

Lemma 5.9. Let $\lambda \geq 1$ and $c_{1}, c_{2}>0$ such that $c_{1} c_{2} \geq 1$ and $\alpha \in \mathbb{R}^{2}$. Define

$$
\begin{aligned}
& R_{c_{1}, c_{2}}^{\alpha}=\left\{(\xi, \eta) \in \mathbb{R}^{2}|| \xi\left|\leq c_{1},\right| \eta \mid \leq c_{2}\right\}-\alpha, \\
& \tilde{R}_{c_{1}, c_{2}}^{\alpha}=\left\{k \in M\left(\mathbb{Z}^{2} / \lambda\right) \cap R_{\ell, w}^{\alpha}\right\} .
\end{aligned}
$$

Then, we have $\sup _{\alpha \in \mathbb{R}^{2}} \# \tilde{R}_{c_{1}, c_{2}}^{\alpha} \lesssim \lambda^{2} c_{1} c_{2}$. 
Lemma 5.10. For $i=1,2,3$, assume (59) and $\min _{i=1,2,3} \# \operatorname{supp}_{k} g_{i} \lesssim P$. Then, we have

$$
\begin{aligned}
& \left|\int_{*} g_{1}\left(\tau_{1}, \ell_{1}\right) g_{2}\left(\tau_{2}, \ell_{2}\right) g_{3}\left(\tau_{3}, \ell_{3}\right)\left(d \tilde{\sigma}_{1}\right)_{\lambda}\left(d \tilde{\sigma}_{2}\right)_{\lambda}\right| \\
\lesssim & \left(P L_{\min }\right)^{\frac{1}{2}} / \lambda\left\|g_{1}\right\|_{L_{\tau}^{2} L_{(d \ell)_{\lambda}}^{2}}\left\|g_{2}\right\|_{L_{\tau}^{2} L_{(d \ell)_{\lambda}}^{2}}\left\|g_{3}\right\|_{L_{\tau}^{2} L_{(d \ell)_{\lambda}}^{2}} .
\end{aligned}
$$

We turn to (58) in the case (I). We divide the proof into the two cases (Ia) $\left|\sin \angle\left(\ell_{1}, \ell_{2}\right)\right| \gtrsim 1$ and $(\mathrm{Ib})\left|\sin \angle\left(\ell_{1}, \ell_{2}\right)\right| \ll 1$.

Let us consider the case (Ia) first. It should be noted that in this case we can assume $N_{1} \sim N_{2} \sim N_{3}$. We introduce the Whitney decomposition of $\mathbb{R}^{2} \times \mathbb{R}^{2}$ into square tiles.

Definition 5.11 (Whitney type decomposition). Let $A \geq 2^{10}$ be dyadic, $m \in \mathbb{Z}^{2}$ and set

$$
\begin{aligned}
\mathcal{T}_{m}^{A}=\left\{(\xi, \eta) \in \mathbb{R}^{2} \mid(\xi, \eta) \in\left[m_{1} / A,\left(m_{1}+1\right) / A\right)\right) & \\
& \left.\left.\times\left[m_{2} / A,\left(m_{2}+1\right) / A\right)\right)\right\}, \\
\Phi\left(\xi_{1}, \eta_{1}, \xi_{2}, \eta_{2}\right)= & \xi_{1} \xi_{2}\left(\xi_{1}+\xi_{2}\right)+\eta_{1} \eta_{2}\left(\eta_{1}+\eta_{2}\right), \\
F\left(\xi_{1}, \eta_{1}, \xi_{2}, \eta_{2}\right)= & \xi_{1} \eta_{2}+\xi_{2} \eta_{1}+2\left(\xi_{1} \eta_{1}+\xi_{2} \eta_{2}\right) .
\end{aligned}
$$

We define

$$
\begin{aligned}
& Z_{A}^{1}=\left\{\left(k_{1}, k_{2}\right) \in \mathbb{Z}^{2} \times \mathbb{Z}^{2}|| \Phi\left(\xi_{1}, \eta_{1}, \xi_{2}, \eta_{2}\right) \mid \geq A^{-1} N_{1}^{3} \text { for any }\left(\xi_{j}, \eta_{j}\right) \in \mathcal{T}_{k_{j}}^{A}\right\}, \\
& Z_{A}^{2}=\left\{\left(k_{1}, k_{2}\right) \in \mathbb{Z}^{2} \times \mathbb{Z}^{2}|| F\left(\xi_{1}, \eta_{1}, \xi_{2}, \eta_{2}\right) \mid \geq A^{-1} N_{1}^{2} \text { for any }\left(\xi_{j}, \eta_{j}\right) \in \mathcal{T}_{k_{j}}^{A}\right\}, \\
& Z_{A}=Z_{A}^{1} \cup Z_{A}^{2} \subset \mathbb{Z}^{2} \times \mathbb{Z}^{2}, \quad R_{A}=\bigcup_{\left(k_{1}, k_{2}\right) \in Z_{A}} \mathcal{T}_{k_{1}}^{A} \times \mathcal{T}_{k_{2}}^{A} \subset \mathbb{R}^{2} \times \mathbb{R}^{2} .
\end{aligned}
$$

It is clear that $A_{1} \leq A_{2} \Longrightarrow R_{A_{1}} \subset R_{A_{2}}$. Further, we define

$$
Q_{A}= \begin{cases}R_{A} \backslash R_{A / 2} & \text { for } A \geq 2^{11}, \\ R_{2^{10}} & \text { for } A=2^{10} .\end{cases}
$$

and a set of pairs of integer coordinates $Z_{A}^{\prime} \subset Z_{A}$ such that

$$
\bigcup_{\left(k_{1}, k_{2}\right) \in Z_{A}^{\prime}} \mathcal{T}_{k_{1}}^{A} \times \mathcal{T}_{k_{2}}^{A}=Q_{A} .
$$

We easily see that $Z_{A}^{\prime}$ is uniquely defined and

$$
A_{1} \neq A_{2} \Longrightarrow Q_{A_{1}} \cap Q_{A_{2}}=\emptyset, \quad \bigcup_{2^{10} \leq A \leq A_{0}} Q_{A}=R_{A_{0}}
$$

where $A_{0} \geq 2^{10}$ is dyadic. Thus, we can decompose $\mathbb{R}^{2} \times \mathbb{R}^{2}$ as

$$
\mathbb{R}^{2} \times \mathbb{R}^{2}=\left(\bigcup_{2^{10} \leq A \leq A_{0}} Q_{A}\right) \cup\left(R_{A_{0}}\right)^{c} .
$$

Lastly, we define

$$
\begin{aligned}
\mathcal{A} & =\left\{\left(\tau_{1}, \xi_{1}, \eta_{1}\right) \times\left(\tau_{2}, \xi_{2}, \eta_{2}\right) \in \mathbb{R}^{3} \times \mathbb{R}^{3}|| \sin \angle\left(\left(\xi_{1}, \eta_{1}\right),\left(\xi_{2}, \eta_{2}\right)\right) \mid \gtrsim 1\right\}, \\
\tilde{Z}_{A} & =\left\{\left(k_{1}, k_{2}\right) \in Z_{A}^{\prime} \mid\left(\tilde{\mathcal{T}}_{k_{1}}^{A} \times \tilde{\mathcal{T}}_{k_{2}}^{A}\right) \cap\left(\tilde{G}_{N_{1}, L_{1}} \times \tilde{G}_{N_{2}, L_{2}}\right) \cap \mathcal{A} \neq \emptyset\right\} .
\end{aligned}
$$


Proposition 5.12. Let $\lambda \geq 1$ and $1 \leq A \leq N_{1}$. Assume that $1 \ll N_{3} \lesssim N_{2} \leq N_{1}$, $L_{\text {med }} \leq N_{1}^{2},\left(k_{1}, k_{2}\right) \in \tilde{Z}_{A}$ and (59). Then, we have

$$
\begin{aligned}
& \left.\left|\int_{*} g_{1}\right|_{\tilde{\mathcal{T}}_{k_{1}}^{A}}\left(\tau_{1}, \ell_{1}\right) g_{2}\right|_{\tilde{\mathcal{T}}_{k_{2}}^{A}}\left(\tau_{2}, \ell_{2}\right) g_{3}\left(\tau_{3}, \ell_{3}\right)\left(d \tilde{\sigma}_{1}\right)_{\lambda}\left(d \tilde{\sigma}_{2}\right)_{\lambda} \mid \\
& \lesssim L_{\min }^{\frac{1}{2}}\left(A^{-\frac{1}{2}} N_{1}^{-\frac{1}{2}} L_{\max }^{\frac{1}{2}}+\left\langle A^{\frac{1}{2}} N_{1}^{-1} L_{\max }^{\frac{1}{2}}\right\rangle\right) \\
& \quad\left\|\left.g_{1}\right|_{\tilde{\mathcal{T}}_{k_{1}}^{A}}\right\|_{L_{\tau}^{2} L_{(d \ell)_{\lambda}}^{2}}\left\|\left.g_{2}\right|_{\tilde{\mathcal{T}}_{k_{2}}^{A}}\right\|_{L_{\tau}^{2} L_{(d \ell)_{\lambda}}^{2}}\left\|g_{3}\right\|_{L_{\tau}^{2} L_{(d \ell)_{\lambda}}^{2}} .
\end{aligned}
$$

Proof. For $\left(\xi_{1}, \eta_{1}\right) \times\left(\xi_{2}, \eta_{2}\right) \in \mathcal{T}_{k_{1}}^{A} \times \mathcal{T}_{k_{2}}^{A}$, it holds either $\left|\Phi\left(\xi_{1}, \eta_{1}, \xi_{2}, \eta_{2}\right)\right| \geq A^{-1} N_{1}^{3}$ or $\left|F\left(\xi_{1}, \eta_{1}, \xi_{2}, \eta_{2}\right)\right| \geq A^{-1} N_{1}^{2}$. If $\left|\Phi\left(\xi_{1}, \eta_{1}, \xi_{2}, \eta_{2}\right)\right| \geq A^{-1} N_{1}^{3}$, by using Lemma 5.10 with $P=\lambda^{2} A^{-2} N_{1}^{2}$, we obtain

$$
\begin{aligned}
& \left.\left|\int_{*} g_{1}\right|_{\tilde{\mathcal{T}}_{k_{1}}^{A}}\left(\tau_{1}, \ell_{1}\right) g_{2}\right|_{\tilde{\mathcal{T}}_{k_{2}}^{A}}\left(\tau_{2}, \ell_{2}\right) g_{3}\left(\tau_{3}, \ell_{3}\right)\left(d \tilde{\sigma}_{1}\right)_{\lambda}\left(d \tilde{\sigma}_{2}\right)_{\lambda} \mid \\
\lesssim & A^{-\frac{1}{2}} N_{1}^{-\frac{1}{2}} L_{\min }^{\frac{1}{2}} L_{\max }^{\frac{1}{2}}\left\|\left.g_{1}\right|_{\tilde{\mathcal{T}}_{k_{1}}^{A}}\right\|_{L_{\tau}^{2} L_{(d \ell)_{\lambda}}^{2}}\left\|\left.g_{2}\right|_{\tilde{\mathcal{T}}_{k_{2}}^{A}}\right\|_{L_{\tau}^{2} L_{(d \ell)_{\lambda}}^{2}}\left\|g_{3}\right\|_{L_{\tau}^{2} L_{(d \ell)_{\lambda}}^{2}} .
\end{aligned}
$$

Next we assume $\left|F\left(\xi_{1}, \eta_{1}, \xi_{2}, \eta_{2}\right)\right| \geq A^{-1} N_{1}^{2}$. This case is handled by Proposition 5.8. Note that the assumption $\left|\sin \angle\left(\left(\xi_{1}, \eta_{1}\right),\left(\xi_{2}, \eta_{2}\right)\right)\right| \gtrsim 1$ implies $\left|\xi_{1} \eta_{2}-\xi_{2} \eta_{1}\right| \gtrsim$ $N_{1}^{2}$ which means (61) for $\left(\xi_{1}, \eta_{1}\right) \times\left(\xi_{2}, \eta_{2}\right) \in \mathcal{T}_{k_{1}}^{A} \times \mathcal{T}_{k_{2}}^{A}$. Since $\mathcal{T}_{k}^{A}$ is a square tile whose side length is $A^{-1} N_{1}$, after performing harmless decompositions, $\operatorname{supp}_{k} g_{i}$ is confined in a ball such that its radius is $r \ll A^{-1} N_{1}$, which provides (60). Consequently, because $L_{\text {med }} \leq N_{1}^{2}$, the claim follows from Proposition 5.8.

In the following we recall the almost orthogonal decompositions from [17].

Definition 5.13. Let $\mathcal{K}_{0}, \mathcal{K}_{1}, \mathcal{K}_{2}, \mathcal{K}_{0}^{\prime}, \mathcal{K}_{1}^{\prime}, \mathcal{K}_{2}^{\prime} \subset \mathbb{R}^{2}$ and $\tilde{\mathcal{K}}_{0}, \tilde{\mathcal{K}}_{1}, \tilde{\mathcal{K}}_{2}, \tilde{\mathcal{K}}_{0}^{\prime}, \tilde{\mathcal{K}}_{1}^{\prime}$, $\tilde{\mathcal{K}}_{2}^{\prime} \subset \mathbb{R}^{3}$ be defined as follows:

$$
\begin{aligned}
& \mathcal{K}_{0}=\left\{(\xi, \eta) \in \mathbb{R}^{2}|| \eta-(\sqrt{2}-1)^{\frac{4}{3}} \xi \mid \leq 2^{-20} N_{1}\right\}, \\
& \mathcal{K}_{1}=\left\{(\xi, \eta) \in \mathbb{R}^{2}|| \eta-(\sqrt{2}+1)^{\frac{2}{3}}(\sqrt{2}+\sqrt{3}) \xi \mid \leq 2^{-20} N_{1}\right\}, \\
& \mathcal{K}_{2}=\left\{(\xi, \eta) \in \mathbb{R}^{2}|| \eta+(\sqrt{2}+1)^{\frac{2}{3}}(\sqrt{3}-\sqrt{2}) \xi \mid \leq 2^{-20} N_{1}\right\}, \\
& \mathcal{K}_{0}^{\prime}=\left\{(\xi, \eta) \in \mathbb{R}^{2} \mid(\eta, \xi) \in \mathcal{K}_{0}\right\}, \\
& \mathcal{K}_{1}^{\prime}=\left\{(\xi, \eta) \in \mathbb{R}^{2} \mid(\eta, \xi) \in \mathcal{K}^{1}\right\}, \\
& \mathcal{K}_{2}^{\prime}=\left\{(\xi, \eta) \in \mathbb{R}^{2} \mid(\eta, \xi) \in \mathcal{K}^{2}\right\}, \\
& \tilde{\mathcal{K}}_{j}=\mathbb{R} \times \mathcal{K}_{j}, \quad \tilde{\mathcal{K}}_{j}^{\prime}=\mathbb{R} \times \mathcal{K}_{j}^{\prime} \text { for } j=0,1,2 .
\end{aligned}
$$

We define the subsets of $\mathbb{R}^{2} \times \mathbb{R}^{2}$ and $\mathbb{R}^{3} \times \mathbb{R}^{3}$ as

$$
\begin{aligned}
& \mathcal{K}=\left(\mathcal{K}_{0} \times\left(\mathcal{K}_{1} \cup \mathcal{K}_{2}\right)\right) \cup\left(\left(\mathcal{K}_{1} \cup \mathcal{K}_{2}\right) \times \mathcal{K}_{0}\right) \subset \mathbb{R}^{2} \times \mathbb{R}^{2}, \\
& \tilde{\mathcal{K}}=\left(\tilde{\mathcal{K}}_{0} \times\left(\tilde{\mathcal{K}}_{1} \cup \tilde{\mathcal{K}}_{2}\right)\right) \cup\left(\left(\tilde{\mathcal{K}}_{1} \cup \tilde{\mathcal{K}}_{2}\right) \times \tilde{\mathcal{K}}_{0}\right) \subset \mathbb{R}^{3} \times \mathbb{R}^{3}, \\
& \mathcal{K}^{\prime}=\left(\mathcal{K}_{0}^{\prime} \times\left(\mathcal{K}_{1}^{\prime} \cup \mathcal{K}_{2}^{\prime}\right)\right) \cup\left(\left(\mathcal{K}_{1}^{\prime} \cup \mathcal{K}_{2}^{\prime}\right) \times \mathcal{K}_{0}^{\prime}\right) \subset \mathbb{R}^{2} \times \mathbb{R}^{2}, \\
& \tilde{\mathcal{K}}^{\prime}=\left(\tilde{\mathcal{K}}_{0}^{\prime} \times\left(\tilde{\mathcal{K}}_{1}^{\prime} \cup \tilde{\mathcal{K}}_{2}^{\prime}\right)\right) \cup\left(\left(\tilde{\mathcal{K}}_{1}^{\prime} \cup \tilde{\mathcal{K}}_{2}^{\prime}\right) \times \tilde{\mathcal{K}}_{0}^{\prime}\right) \subset \mathbb{R}^{3} \times \mathbb{R}^{3},
\end{aligned}
$$

and the complementary sets as

$$
\begin{aligned}
(\mathcal{K})^{c} & =\left(\mathbb{R}^{2} \times \mathbb{R}^{2}\right) \backslash \mathcal{K}, & & (\tilde{\mathcal{K}})^{c}=\left(\mathbb{R}^{3} \times \mathbb{R}^{3}\right) \backslash \tilde{\mathcal{K}} \\
\left(\mathcal{K}^{\prime}\right)^{c} & =\left(\mathbb{R}^{2} \times \mathbb{R}^{2}\right) \backslash \mathcal{K}^{\prime}, & & \left(\tilde{\mathcal{K}}^{\prime}\right)^{c}=\left(\mathbb{R}^{3} \times \mathbb{R}^{3}\right) \backslash \tilde{\mathcal{K}}^{\prime} .
\end{aligned}
$$


Lastly, we define

$$
\widehat{Z}_{A}=\left\{\left(k_{1}, k_{2}\right) \in \tilde{Z}_{A} \mid\left(\mathcal{T}_{k_{1}}^{A} \times \mathcal{T}_{k_{2}}^{A}\right) \cap\left((\mathcal{K})^{c} \cap\left(\mathcal{K}^{\prime}\right)^{c}\right) \neq \emptyset\right\},
$$

and $\bar{Z}_{A}$ as the collection of $\left(k_{1}, k_{2}\right) \in \mathbb{Z}^{2} \times \mathbb{Z}^{2}$ which satisfies

$$
\begin{gathered}
\mathcal{T}_{k_{1}}^{A} \times \mathcal{T}_{k_{2}}^{A} \not \subset \bigcup_{2^{100} \leq A^{\prime} \leq A} \bigcup_{\left(k_{1}^{\prime}, k_{2}^{\prime}\right) \in \widehat{Z}_{A}}\left(\mathcal{T}_{k_{1}^{\prime}}^{A^{\prime}} \times \mathcal{T}_{k_{2}^{\prime}}^{A^{\prime}}\right), \\
\left(\tilde{\mathcal{T}}_{k_{1}}^{A} \times \tilde{\mathcal{T}}_{k_{2}}^{A}\right) \cap\left(\tilde{G}_{N_{1}, L_{1}} \times \tilde{G}_{N_{2}, L_{2}}\right) \cap \mathcal{A} \cap\left((\tilde{\mathcal{K}})^{c} \cap\left(\tilde{\mathcal{K}}^{\prime}\right)^{c}\right) \neq \emptyset .
\end{gathered}
$$

Lemma 5.14 ([17, Lemma 3.7, p. 17]). For fixed $k_{1} \in \mathbb{Z}^{2}$, the number of $k_{2} \in \mathbb{Z}^{2}$ such that $\left(k_{1}, k_{2}\right) \in \widehat{Z}_{A}$ is less than $2^{1000}$. Furthermore, the same claim holds true if we replace $\widehat{Z}_{A}$ by $\bar{Z}_{A}$.

We show (58) under the assumption $\left(\ell_{1}, \ell_{2}\right) \in(\mathcal{K})^{c} \cap\left(\mathcal{K}^{\prime}\right)^{c}$.

Proof of (58) for the case $\left(\ell_{1}, \ell_{2}\right) \in(\mathcal{K})^{c} \cap\left(\mathcal{K}^{\prime}\right)^{c}$. By the definitions of $\widehat{Z}_{A}$ and $\bar{Z}_{A_{0}}$, we see that the set $\left(\tilde{G}_{N_{1}, L_{1}} \times \tilde{G}_{N_{2}, L_{2}}\right) \cap \mathcal{A} \cap(\tilde{\mathcal{K}})^{c} \cap\left(\tilde{\mathcal{K}}^{\prime}\right)^{c}$ is contained in

$$
\bigcup_{2^{10} \leq A \leq N_{1}} \bigcup_{\left(k_{1}, k_{2}\right) \in \widehat{Z}_{A}}\left(\tilde{\mathcal{T}}_{k_{1}}^{A} \times \tilde{\mathcal{T}}_{k_{2}}^{A}\right) \cup \bigcup_{\left(k_{1}, k_{2}\right) \in \bar{Z}_{N_{1}}}\left(\tilde{\mathcal{T}}_{k_{1}}^{N_{1}} \times \tilde{\mathcal{T}}_{k_{2}}^{N_{1}}\right) .
$$

For short, we use

$$
I_{A}^{k_{1}, k_{2}}=\left.\left|\int_{*} g_{1}\right|_{\tilde{\mathcal{T}}_{k_{1}}^{A}}\left(\tau_{1}, \ell_{1}\right) g_{2}\right|_{\tilde{\mathcal{T}}_{k_{2}}^{A}}\left(\tau_{2}, \ell_{2}\right) g_{3}\left(\tau_{3}, \ell_{3}\right)\left(d \tilde{\sigma}_{1}\right)_{\lambda}\left(d \tilde{\sigma}_{2}\right)_{\lambda} \mid
$$

It is observed that

$$
\begin{aligned}
& \left|\int_{*} g_{1}\left(\tau_{1}, \ell_{1}\right) g_{2}\left(\tau_{2}, \ell_{2}\right) g_{3}\left(\tau_{3}, \ell_{3}\right)\left(d \sigma_{1}\right)_{\lambda}\left(d \sigma_{2}\right)_{\lambda}\right| \\
\lesssim & \sum_{2^{10} \leq A \leq N_{1}} \sum_{\left(k_{1}, k_{2}\right) \in \widehat{Z}_{A}} I_{A}^{k_{1}, k_{2}}+\sum_{\left(k_{1}, k_{2}\right) \in \bar{Z}_{N_{1}}} I_{N_{1}}^{k_{1}, k_{2}} .
\end{aligned}
$$

For the former term, since $N_{1} \sim N_{2} \sim N_{3}$, by employing Proposition 5.12 and Lemma 5.14, we get

$$
\begin{aligned}
& \sum_{2^{10} \leq A \leq N_{1}} \sum_{\left(k_{1}, k_{2}\right) \in \widehat{Z}_{A}} I_{A}^{k_{1}, k_{2}} \\
\lesssim & \sum_{2^{10} \leq A \leq N_{1}} L_{\min }^{\frac{1}{2}}\left(A^{-\frac{1}{2}} N_{1}^{-\frac{1}{2}} L_{\max }^{\frac{1}{2}}+\left\langle A^{\frac{1}{2}} N_{1}^{-1} L_{\max }^{\frac{1}{2}}\right\rangle\right) \\
& \times \sum_{\left(k_{1}, k_{2}\right) \in \widehat{Z}_{A}}\left\|\left.g_{1}\right|_{\tilde{\mathcal{T}}_{k_{1}}^{A}}\right\|_{L_{\tau}^{2} L_{(d \ell)_{\lambda}}^{2}}\left\|\left.g_{2}\right|_{\tilde{\mathcal{T}}_{k_{2}}^{A}}\right\|_{L_{\tau}^{2} L_{(d \ell)_{\lambda}}^{2}}\left\|g_{3}\right\|_{L_{\tau}^{2} L_{(d \ell)_{\lambda}}^{2}} \\
\lesssim & \left(\log N_{3}\right) L_{\min }^{\frac{1}{2}}\left\langle N_{1}^{-\frac{1}{2}} L_{\max }^{\frac{1}{2}}\right\rangle\left\|g_{1}\right\|_{L_{\tau}^{2} L_{(d \ell)_{\lambda}}^{2}}\left\|g_{2}\right\|_{L_{\tau}^{2} L_{(d \ell)_{\lambda}}^{2}}\left\|g_{3}\right\|_{L_{\tau}^{2} L_{(d \ell)_{\lambda}}^{2}} .
\end{aligned}
$$

For the latter term, it follows from Lemma 5.10 with $M \sim \lambda^{2}$ and Lemma 5.14 that

$$
\sum_{\left(k_{1}, k_{2}\right) \in \bar{Z}_{N_{1}}} I_{N_{1}}^{k_{1}, k_{2}} \lesssim L_{\min }^{\frac{1}{2}}\left\|g_{1}\right\|_{L_{\tau}^{2} L_{(d \ell)_{\lambda}}^{2}}\left\|g_{2}\right\|_{L_{\tau}^{2} L_{(d \ell)_{\lambda}}^{2}}\left\|g_{3}\right\|_{L_{\tau}^{2} L_{(d \ell)_{\lambda}}^{2}}
$$

which completes the proof. 
Next we prove the estimate $(58)$ for $\left(\ell_{1}, \ell_{2}\right) \in\left(\mathcal{K} \cup \mathcal{K}^{\prime}\right)$. In this case, the almost one-to-one correspondence of $\left(k_{1}, k_{2}\right) \in \tilde{Z}_{A}$ does not hold. Therefore, we need to introduce another decomposition. We note that, by exchanging the roles of $\ell_{i, 1}$ and $\ell_{i, 2}$ with $i=1,2$, once the estimate (58) is verified for the case $\left(\ell_{1}, \ell_{2}\right) \in \mathcal{K}$, one can obtain the same estimate for $\left(\ell_{1}, \ell_{2}\right) \in \mathcal{K}^{\prime}$. For the same reason, it suffices to show the estimate $(58)$ for the case $\left(\ell_{1}, \ell_{2}\right) \in\left(\mathcal{K}_{1} \cup \mathcal{K}_{2}\right) \times \mathcal{K}_{0}$.

Definition 5.15. Let $m=(n, z) \in \mathbb{N} \times \mathbb{Z}$. We define the monotone increasing sequence $\left\{a_{A, n}\right\}_{n \in \mathbb{N}}$ as

$$
a_{A, 1}=0, \quad a_{A, n+1}=a_{A, n}+\frac{N_{1}}{\sqrt{(n+1) A}} .
$$

and sets $\mathcal{R}_{A, m, 1}, \mathcal{R}_{A, m, 2}$ as follows:

$$
\begin{aligned}
& \mathcal{R}_{A, m, 1}=\left\{\begin{array}{l|l}
(\xi, \eta) \in \mathbb{R}^{2} & \begin{array}{l}
a_{A, n} \leq\left|\eta-(\sqrt{2}+1)^{\frac{2}{3}}(\sqrt{2}+\sqrt{3}) \xi\right|<a_{A, n+1}, \\
z A^{-1} N_{1} \leq \eta-(\sqrt{2}+1)^{\frac{2}{3}} \xi<(z+1) A^{-1} N_{1}
\end{array}
\end{array}\right\}, \\
& \mathcal{R}_{A, m, 2}=\left\{\begin{array}{l|l}
(\xi, \eta) \in \mathbb{R}^{2} & \begin{array}{l}
a_{A, n} \leq\left|\eta+(\sqrt{2}+1)^{\frac{2}{3}}(\sqrt{3}-\sqrt{2}) \xi\right|<a_{A, n+1}, \\
z A^{-1} N_{1} \leq \eta-(\sqrt{2}+1)^{\frac{2}{3}} \xi<(z+1) A^{-1} N_{1}
\end{array}
\end{array}\right\}, \\
& \tilde{\mathcal{R}}_{A, m, 1}=\mathbb{R} \times \mathcal{R}_{A, m, 1}, \quad \tilde{\mathcal{R}}_{A, m, 2}=\mathbb{R} \times \mathcal{R}_{A, m, 2} .
\end{aligned}
$$

We will perform the Whitney type decomposition by using the above sets instead of simple square tiles. We define for $i=1,2$ that

$$
\begin{aligned}
& M_{A, i}^{1}=\left\{\begin{array}{l|l}
(m, k) \in(\mathbb{N} \times \mathbb{Z}) \times \mathbb{Z}^{2} & \begin{array}{l}
\left|\Phi\left(\xi_{1}, \eta_{1}, \xi_{2}, \eta_{2}\right)\right| \geq A^{-1} N_{1}^{3} \\
\text { for any }\left(\xi_{1}, \eta_{1}\right) \in \mathcal{R}_{A, m, i} \text { and }\left(\xi_{2}, \eta_{2}\right) \in \mathcal{T}_{k}^{A}
\end{array}
\end{array}\right\}, \\
& M_{A, i}^{2}=\left\{\begin{array}{l|l}
(m, k) \in(\mathbb{N} \times \mathbb{Z}) \times \mathbb{Z}^{2} & \begin{array}{l}
\left|F\left(\xi_{1}, \eta_{1}, \xi_{2}, \eta_{2}\right)\right| \geq A^{-1} N_{1}^{3} \\
\text { for any }\left(\xi_{1}, \eta_{1}\right) \in \mathcal{R}_{A, m, i} \text { and }\left(\xi_{2}, \eta_{2}\right) \in \mathcal{T}_{k}^{A}
\end{array}
\end{array}\right\}, \\
& M_{A, i}=M_{A, i}^{1} \cup M_{A, i}^{2} \subset(\mathbb{N} \times \mathbb{Z}) \times \mathbb{Z}^{2}, \\
& R_{A, i}=\bigcup_{(m, k) \in M_{A, i}} \mathcal{R}_{A, m, i} \times \mathcal{T}_{k}^{A} \subset \mathbb{R}^{2} \times \mathbb{R}^{2} \text {. }
\end{aligned}
$$

Furthermore, we define $M_{A, i}^{\prime} \subset M_{A, i}$ as the collection of $(m, k) \in \mathbb{N} \times \mathbb{Z}$ such that

$$
\mathcal{R}_{A, m, i} \times \mathcal{T}_{k}^{A} \subset \bigcup_{2^{10} \leq A^{\prime}<A} R_{A^{\prime}, i}
$$

By using $M_{A, i}^{\prime}$, we define

$$
Q_{A, i}=\left\{\begin{array}{cc}
R_{A, i} \backslash \bigcup_{(m, k) \in M_{A, i}^{\prime}}\left(\mathcal{R}_{A, m, i} \times \mathcal{T}_{k}^{A}\right) & \text { for } A \geq 2^{11}, \\
R_{2^{10}, i} & \text { for } A=2^{10},
\end{array}\right.
$$

and $\tilde{M}_{A, i}=M_{A, i} \backslash M_{A, i}^{\prime}$. Clearly, the followings hold.

$$
\bigcup_{(m, k) \in \tilde{M}_{A, i}} \mathcal{R}_{A, m, i} \times \mathcal{T}_{k}^{A}=Q_{A, i}, \quad \bigcup_{2^{10} \leq A \leq A_{0}} Q_{A, i}=R_{A_{0}, i}
$$


where $A_{0} \geq 2^{10}$ is dyadic. Lastly, we define

$$
\begin{aligned}
& \widehat{Z}_{A, i}=\left\{(m, k) \in \tilde{M}_{A, i} \mid\left(\tilde{\mathcal{R}}_{A, m, i} \times \tilde{\mathcal{T}}_{k}^{A}\right) \cap\left(\tilde{G}_{N_{1}, L_{1}} \times \tilde{G}_{N_{2}, L_{2}}\right) \cap\left(\tilde{\mathcal{K}}_{i} \times \tilde{\mathcal{K}}_{0}\right) \neq \emptyset\right\}, \\
& \bar{Z}_{A, i}=\left\{(m, k) \in M_{A, i}^{c} \mid\left(\tilde{\mathcal{R}}_{A, m, i} \times \tilde{\mathcal{T}}_{k}^{A}\right) \cap\left(\tilde{G}_{N_{1}, L_{1}} \times \tilde{G}_{N_{2}, L_{2}}\right) \cap\left(\tilde{\mathcal{K}}_{i} \times \tilde{\mathcal{K}}_{0}\right) \neq \emptyset\right\},
\end{aligned}
$$

where $M_{A, i}^{c}=(\mathbb{N} \times \mathbb{Z}) \backslash M_{A, i}$. We easily see that

$$
\left(\tilde{G}_{N_{1}, L_{1}} \times \tilde{G}_{N_{2}, L_{2}}\right) \cap\left(\tilde{\mathcal{K}}_{i} \times \tilde{\mathcal{K}}_{0}\right) \subset \bigcup_{(m, k) \in \widehat{Z}_{A, i}}\left(\tilde{\mathcal{R}}_{A, m, i} \times \tilde{\mathcal{T}}_{k}^{A}\right) \cup \bigcup_{(m, k) \in \bar{Z}_{A, i}}\left(\tilde{\mathcal{R}}_{A, m, i} \times \tilde{\mathcal{T}}_{k}^{A}\right) .
$$

Lemma 5.16 ([17, Lemma 3.9, p. 26]). Let $i=1,2$. For fixed $m \in \mathbb{N} \times \mathbb{Z}$, the number of $k \in \mathbb{Z}^{2}$ such that $(m, k) \in \widehat{Z}_{A, i}$ is less than $2^{1000}$. On the other hand, for fixed $k \in \mathbb{Z}^{2}$, the number of $m \in \mathbb{N} \times \mathbb{Z}$ such that $(m, k) \in \widehat{Z}_{A, i}$ is less than $2^{1000}$. Furthermore, the claim holds true if we replace $\widehat{Z}_{A, i}$ by $\bar{Z}_{A, i}$ in the above statements.

We establish (58) under the case $\left(\ell_{1}, \ell_{2}\right) \in\left(\mathcal{K}_{1} \cup \mathcal{K}_{2}\right) \times \mathcal{K}_{0}$. To avoid redundancy, here we treat only the case $\left(\ell_{1}, \ell_{2}\right) \in \mathcal{K}_{1} \times \mathcal{K}_{0}$.

Proof of (58) for the case $\left(\ell_{1}, \ell_{2}\right) \in \mathcal{K}_{1} \times \mathcal{K}_{0}$. The strategy of the proof is the same as that for the case $\left(\ell_{1}, \ell_{2}\right) \in(\mathcal{K})^{c} \cap\left(\mathcal{K}^{\prime}\right)^{c}$. Let us write

$$
I_{A}^{m, k}=\left.\left|\int_{*} g_{1}\right|_{\tilde{\mathcal{R}}_{A, m, 1}}\left(\tau_{1}, \ell_{1}\right) g_{2}\right|_{\tilde{\mathcal{T}}_{k}^{A}}\left(\tau_{2}, \ell_{2}\right) g_{3}\left(\tau_{3}, \ell_{3}\right)\left(d \tilde{\sigma}_{1}\right)_{\lambda}\left(d \tilde{\sigma}_{2}\right)_{\lambda} \mid .
$$

By the definitions of $\widehat{Z}_{A, 1}$ and $\bar{Z}_{A, 1}$, we observe that

$$
\begin{aligned}
& \left|\int_{*} g_{1}\left(\tau_{1}, \ell_{1}\right) g_{2}\left(\tau_{2}, \ell_{2}\right) g_{3}\left(\tau_{3}, \ell_{3}\right)\left(d \tilde{\sigma}_{1}\right)_{\lambda}\left(d \tilde{\sigma}_{2}\right)_{\lambda}\right| \\
& \lesssim \sum_{2^{10} \leq A \leq N_{1}} \sum_{(m, k) \in \widehat{Z}_{A}} I_{A}^{m, k}+\sum_{(m, k) \in \bar{Z}_{N_{1}}} I_{N_{1}}^{m, k} .
\end{aligned}
$$

As in the proof for the case $\left(\ell_{1}, \ell_{2}\right) \in(\mathcal{K})^{c} \cap\left(\mathcal{K}^{\prime}\right)^{c}$, the first term is estimated by Proposition 5.12 and Lemma 5.16 , and the second is estimated by Lemmas 5.10 and 5.16. We omit the details.

Next we show (58) for the case (Ib) $\left|\sin \angle\left(\ell_{1}, \ell_{2}\right)\right| \ll 1$. This case requires an angular decomposition. We cover the unit circle with the sets

$$
\Theta_{j}^{A}=\left[\frac{\pi}{A}(j-2), \frac{\pi}{A}(j+2)\right] \cup\left[-\pi+\frac{\pi}{A}(j-2),-\pi+\frac{\pi}{A}(j+2)\right] .
$$

Angles from these sets give rise to the following covering of the plane:

$$
\mathfrak{D}_{j}^{A}=\left\{r(\cos \theta, \sin \theta) \in \mathbb{R}^{2} \mid \theta \in \Theta_{j}^{A} \text { and } r \in[0, \infty)\right\} .
$$

We set $\tilde{\mathfrak{D}}_{j}^{A}=\mathbb{R} \times \mathfrak{D}_{j}^{A}$.

Recall that it is assumed $\max \left(\left|\ell_{1,1}+\ell_{1,2}\right|,\left|\ell_{2,1}+\ell_{2,2}\right|\right) \geq 2^{-6} N_{1}$, which means $\left(\ell_{1}, \ell_{2}\right) \notin \mathfrak{D}_{2^{9} \times 3}^{2^{11}} \times \mathfrak{D}_{2^{9} \times 3}^{2^{11}}$. The proof is divided into two cases:

Case 1. $\left(\ell_{1}, \ell_{2}\right) \in \bigcup_{\substack{0 \leq j \leq 2^{11}-1 \\ j \neq 0,2^{9} \times 3,2^{10}}}\left(\mathfrak{D}_{j}^{2^{11}} \times \mathfrak{D}_{j}^{2^{11}}\right)$,

Case 2. $\left(\ell_{1}, \ell_{2}\right) \in\left(\mathfrak{D}_{0}^{2^{11}} \times \mathfrak{D}_{0}^{2^{11}}\right) \cup\left(\mathfrak{D}_{2^{10}}^{2^{11}} \times \mathfrak{D}_{2^{10}}^{2^{11}}\right)$. 
We begin with Case 1. It suffices to show (58) under the assumption $\left(\ell_{1}, \ell_{2}\right) \in$ $\mathfrak{D}_{j}^{2^{11}} \times \mathfrak{D}_{j}^{2^{11}}$ with fixed $j \neq 0,2^{9} \times 3,2^{10}$. Further, since $\left|\sin \angle\left(\ell_{1}, \ell_{2}\right)\right| \ll 1$, we may assume

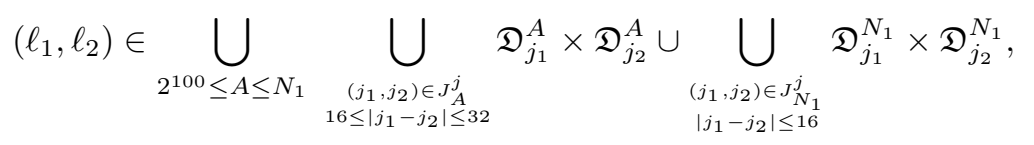

where

$$
J_{A}^{j}=\left\{\left(j_{1}, j_{2}\right) \mid 0 \leq j_{1}, j_{2} \leq A-1,\left(\mathfrak{D}_{j_{1}}^{A} \times \mathfrak{D}_{j_{2}}^{A}\right) \subset \mathfrak{D}_{j}^{2^{11}} \times \mathfrak{D}_{j}^{2^{11}}\right\} .
$$

Proposition 5.17. Let $2^{100} \leq A \leq N_{1}$. Assume that $1 \ll N_{3} \lesssim N_{2} \leq N_{1}$, $L_{\text {med }} \leq N_{1}^{2},\left(j_{1}, j_{2}\right) \in J_{A}^{j}$ such that $16 \leq\left|j_{1}-j_{2}\right| \leq 32$ and (59). Then we have

$$
\begin{aligned}
& \left.\left|\int_{*} g_{1}\right|_{\tilde{\mathfrak{D}}_{j_{1}}^{A}}\left(\tau_{1}, \ell_{1}\right) g_{2}\right|_{\tilde{\mathfrak{D}}_{j_{2}}^{A}}\left(\tau_{2}, \ell_{2}\right) g_{3}\left(\tau_{3}, \ell_{3}\right)\left(d \tilde{\sigma}_{1}\right)_{\lambda}\left(d \tilde{\sigma}_{2}\right)_{\lambda} \mid \\
\lesssim & L_{\min }^{\frac{1}{2}} \bar{C}\left(A, N_{1}, N_{3}, L_{\max }\right)\left\|\left.g_{1}\right|_{\tilde{\mathfrak{D}}_{j_{1}}^{A}}\right\|_{L_{\tau}^{2} L_{(d \ell)_{\lambda}}^{2}}\left\|\left.g_{2}\right|_{\tilde{\mathfrak{D}}_{j_{2}}^{A}}\right\|_{L_{\tau}^{2} L_{(d \ell)_{\lambda}}^{2}}\left\|g_{3}\right\|_{L_{\tau}^{2} L_{(d \ell)_{\lambda}}^{2}},
\end{aligned}
$$

where

$$
\bar{C}\left(A, N_{1}, N_{3}, L_{\max }\right)= \begin{cases}N_{1}^{-1} N_{3}^{\frac{1}{2}} L_{\max }^{\frac{1}{2}} & \text { for } N_{3} \geq 2^{30} A^{-1} N_{1}, \\ \left\langle A^{\frac{1}{2}} N_{1}^{-1} L_{\max }^{\frac{1}{2}}\right\rangle & \text { for } N_{3} \leq 2^{30} A^{-1} N_{1} .\end{cases}
$$

Proof. First we assume $N_{3} \geq 2^{30} A^{-1} N_{1}$. In this case, for $\left(\xi_{1}, \eta_{1}\right) \times\left(\xi_{2}, \eta_{2}\right) \in$ $\mathfrak{D}_{j_{1}}^{A} \times \mathfrak{D}_{j_{2}}^{A}$ a simple calculation yields

$$
\left|\Phi\left(\xi_{1}, \eta_{1}, \xi_{2}, \eta_{2}\right)\right| \gtrsim A^{-1} N_{1}^{3} .
$$

To see this, we put $r_{1}=\left|\left(\xi_{1}, \eta_{1}\right)\right|, r_{2}=\left|\left(\xi_{2}, \eta_{2}\right)\right|$. $\theta_{1}, \theta_{2} \in[0,2 \pi)$ denote angular variables defined by

$$
\left(\xi_{1}, \eta_{1}\right)=r_{1}\left(\cos \theta_{1}, \sin \theta_{1}\right), \quad\left(\xi_{2}, \eta_{2}\right)=r_{2}\left(\cos \theta_{2}, \sin \theta_{2}\right) .
$$

Recall that $\left(\xi_{1}, \eta_{1}\right) \times\left(\xi_{2}, \eta_{2}\right) \notin \mathfrak{D}_{2^{9} \times 3}^{2^{11}} \times \mathfrak{D}_{2^{9} \times 3}^{2^{11}}$ is assumed. Thus without loss of generality, we may assume that $\left(\xi_{1}, \eta_{1}\right) \notin \mathfrak{D}_{2^{9} \times 3}^{2^{11}}$ which provides $\left|\cos \theta_{1}+\sin \theta_{1}\right|=$ $\sqrt{2}\left|\sin \left(\theta_{1}+\pi / 4\right)\right|>2^{-11} \pi$. We deduce from the assumption $\left|j_{1}-j_{2}\right| \leq 32$ that $\left|\left(\cos \theta_{1}, \sin \theta_{1}\right)-\left(\cos \theta_{2}, \sin \theta_{2}\right)\right| \leq 2^{7} A^{-1}$ or $\left|\left(\cos \theta_{1}, \sin \theta_{1}\right)+\left(\cos \theta_{2}, \sin \theta_{2}\right)\right| \leq$ $2^{7} A^{-1}$. If $\left|\left(\cos \theta_{1}, \sin \theta_{1}\right)-\left(\cos \theta_{2}, \sin \theta_{2}\right)\right| \leq 2^{7} A^{-1}$, it is observed that

$$
\begin{aligned}
\left|\Phi\left(\xi_{1}, \eta_{1}, \xi_{2}, \eta_{2}\right)\right| & =\left|\xi_{1} \xi_{2}\left(\xi_{1}+\xi_{2}\right)+\eta_{1} \eta_{2}\left(\eta_{1}+\eta_{2}\right)\right| \\
& \geq r_{1} r_{2}\left(r_{1}+r_{2}\right)\left|\cos ^{3} \theta_{1}+\sin ^{3} \theta_{1}\right|-2^{9} A^{-1} r_{1} r_{2}\left(r_{1}+r_{2}\right) \\
& =r_{1} r_{2}\left(r_{1}+r_{2}\right)\left(1-2^{-1} \sin 2 \theta_{1}\right)\left|\cos \theta_{1}+\sin \theta_{1}\right| \\
& \quad-2^{9} A^{-1} r_{1} r_{2}\left(r_{1}+r_{2}\right) .
\end{aligned}
$$

Clearly, this implies $\left|\Phi\left(\xi_{1}, \eta_{1}, \xi_{2}, \eta_{2}\right)\right| \gtrsim N_{1}^{3}$. Similarly, for the case $\mid\left(\cos \theta_{1}, \sin \theta_{1}\right)+$ $\left(\cos \theta_{2}, \sin \theta_{2}\right) \mid \leq 2^{7} A^{-1}$, we calculate

$$
\begin{aligned}
\left|\Phi\left(\xi_{1}, \eta_{1}, \xi_{2}, \eta_{2}\right)\right| & =\left|\xi_{1} \xi_{2}\left(\xi_{1}+\xi_{2}\right)+\eta_{1} \eta_{2}\left(\eta_{1}+\eta_{2}\right)\right| \\
& \geq r_{1} r_{2}\left(r_{1}-r_{2}\right)\left|\cos ^{3} \theta_{1}+\sin ^{3} \theta_{1}\right|-2^{10} A^{-1} N_{1}^{3} \\
& \geq 2^{-13} r_{1} r_{2}\left(r_{1}-r_{2}\right)-2^{10} A^{-1} N_{1}^{3} .
\end{aligned}
$$


Then it suffices to show $\left|r_{1}-r_{2}\right| \geq 2^{27} A^{-1} N_{1}$. Since $N_{3} \geq 2^{30} A^{-1} N_{1}$, without loss of generality, we can assume $\left|\xi_{1}+\xi_{2}\right|=\left|r_{1} \cos \theta_{1}+r_{2} \cos \theta_{2}\right| \geq 2^{28} A^{-1} N_{1}$. We see

$$
\begin{aligned}
\left|r_{1}-r_{2}\right| & \geq\left|r_{1} \cos \theta_{1}-r_{2} \cos \theta_{1}\right| \\
& \geq\left|r_{1} \cos \theta_{1}+r_{2} \cos \theta_{2}\right|-2^{10} A^{-1} N_{1} \\
& \geq 2^{27} A^{-1} N_{1} .
\end{aligned}
$$

This completes the proof of $\left|\Phi\left(\xi_{1}, \eta_{1}, \xi_{2}, \eta_{2}\right)\right| \gtrsim A^{-1} N_{1}^{3}$ which yields $L_{\max } \gtrsim A^{-1} N_{1}^{3}$. Consequently, it follows from Lemma 5.10 with $P \sim \lambda^{2} A^{-1} N_{1} N_{3}$ that

$$
\begin{aligned}
& \left.\left|\int_{*} g_{1}\right|_{\tilde{\mathfrak{D}}_{j_{1}}^{A}}\left(\tau_{1}, \ell_{1}\right) g_{2}\right|_{\tilde{\mathfrak{D}}_{j_{2}}^{A}}\left(\tau_{2}, \ell_{2}\right) g_{3}\left(\tau_{3}, \ell_{3}\right)\left(d \tilde{\sigma}_{1}\right)_{\lambda}\left(d \tilde{\sigma}_{2}\right)_{\lambda} \mid \\
\lesssim & \left.N_{1}^{-1} N_{3}^{\frac{1}{2}} L_{\min }^{\frac{1}{2}} L_{\max }^{\frac{1}{2}}\left\|\left.g_{1}\right|_{\tilde{\mathfrak{D}}_{j_{1}}^{A}}\right\|\right|_{L_{\tau}^{2} L_{(d \ell)_{\lambda}}^{2}}\left\|\left.g_{2}\right|_{\tilde{\mathfrak{D}}_{j_{2}}^{A}}\right\|_{L_{\tau}^{2} L_{(d \ell)_{\lambda}}^{2}}\left\|g_{3}\right\|_{L_{\tau}^{2} L_{(d \ell)_{\lambda}}^{2}} .
\end{aligned}
$$

Next we assume $N_{3} \leq 2^{30} A^{-1} N_{1}$. This case is treated by Proposition 5.8. To utilize Proposition 5.8, we only need to show

$$
\left|\left(\xi_{1} \eta_{2}-\xi_{2} \eta_{1}\right)\left(\xi_{1} \eta_{2}+\xi_{2} \eta_{1}+2\left(\xi_{1} \eta_{1}+\xi_{2} \eta_{2}\right)\right)\right| \gtrsim A^{-1} N_{1}^{4}
$$

for $\left(\xi_{1}, \eta_{1}\right) \times\left(\xi_{2}, \eta_{2}\right) \in \mathfrak{D}_{j_{1}}^{A} \times \mathfrak{D}_{j_{2}}^{A}$. Since $\left|\xi_{1} \eta_{2}-\xi_{2} \eta_{1}\right| \gtrsim A^{-1} N_{1}^{2}$ is clear, it suffices to show $\left|\xi_{1} \eta_{2}+\xi_{2} \eta_{1}+2\left(\xi_{1} \eta_{1}+\xi_{2} \eta_{2}\right)\right| \gtrsim N_{1}^{2}$. Let us recall

$$
\left(\ell_{1}, \ell_{2}\right) \notin\left(\mathfrak{D}_{0}^{2^{11}} \times \mathfrak{D}_{0}^{2^{11}}\right) \cup\left(\mathfrak{D}_{2^{10}}^{2^{11}} \times \mathfrak{D}_{2^{10}}^{2^{11}}\right),
$$

which suggests that we may assume $\left|\xi_{1} \eta_{1}\right| \geq 2^{-15} N_{1}^{2}$. Thus it is observed that

$$
\begin{aligned}
\left|\xi_{1} \eta_{2}+\xi_{2} \eta_{1}+2\left(\xi_{1} \eta_{1}+\xi_{2} \eta_{2}\right)\right| & \geq 2\left|\xi_{1} \eta_{1}+\xi_{2}\left(\eta_{1}+\eta_{2}\right)\right|-\left|\xi_{1} \eta_{2}-\xi_{2} \eta_{1}\right| \\
& \geq 2^{-14} N_{1}^{2}-2^{3} N_{1} N_{3}-2^{10} A^{-1} N_{1}^{2} \geq 2^{-15} N_{1}^{2} .
\end{aligned}
$$

This completes the proof.

Proof of (58) for the case $\left(\ell_{1}, \ell_{2}\right) \in \mathfrak{D}_{j}^{2^{11}} \times \mathfrak{D}_{j}^{2^{11}}$ with fixed $j \neq 0,2^{9} \times 3,2^{10}$. By using

$$
I_{A}^{j_{1}, j_{2}}=\left.\left|\int_{*} g_{1}\right|_{\tilde{\mathfrak{D}}_{j_{1}}^{A}}\left(\tau_{1}, \ell_{1}\right) g_{2}\right|_{\tilde{\mathfrak{D}}_{j_{2}}^{A}}\left(\tau_{2}, \ell_{2}\right) g_{3}\left(\tau_{3}, \ell_{3}\right)\left(d \tilde{\sigma}_{1}\right)_{\lambda}\left(d \tilde{\sigma}_{2}\right)_{\lambda} \mid
$$

since (62), we can write

$$
\begin{aligned}
& \left.\left|\int_{*} g_{1}\right|_{\tilde{\mathfrak{D}}_{j}^{21}}\left(\tau_{1}, \ell_{1}\right) g_{2}\right|_{\tilde{\mathfrak{D}}_{j}^{211}}\left(\tau_{2}, \ell_{2}\right) g_{3}\left(\tau_{3}, \ell_{3}\right)\left(d \tilde{\sigma}_{1}\right)_{\lambda}\left(d \tilde{\sigma}_{2}\right)_{\lambda} \mid \\
\lesssim & \left(\sum_{2^{100} \leq A \leq 2^{30} N_{1} / N_{3}}+\sum_{2^{30} N_{1} / N_{3} \leq A \leq N_{1}}\right) \sum_{\substack{\left(j_{1}, j_{2}\right) \in J_{A}^{j} \\
16 \leq\left|j_{1}-j_{2}\right| \leq 32}} I_{A}^{j_{1}, j_{2}}+\sum_{\substack{\left(j_{1}, j_{2}\right) \in J_{N_{1}}^{j} \\
\left|j_{1}-j_{2}\right| \leq 16}} I_{N_{1}}^{j_{1}, j_{2}} .
\end{aligned}
$$


SHINYA KINOSHITA AND ROBERT SCHIPPA

For the first term, it should be noted that we may assume $A \geq N_{1} / N_{3}$, otherwise $I_{A}^{j_{1}, j_{2}}$ with $16 \leq\left|j_{1}-j_{2}\right|$ vanishes. By using Proposition 5.17, we obtain

$$
\begin{aligned}
& \left(\sum_{N_{1} / N_{3} \leq A \leq 2^{30} N_{1} / N_{3}}+\sum_{2^{30} N_{1} / N_{3} \leq A \leq N_{1}}\right) \sum_{\substack{\left(j_{1}, j_{2}\right) \in J_{A}^{j} \\
16 \leq\left|j_{1}-j_{2}\right| \leq 32}} I_{A}^{j_{1}, j_{2}} \\
& \lesssim L_{\min }^{\frac{1}{2}}\left(\sum_{A \sim N_{1} / N_{3}}\left\langle A^{\frac{1}{2}} N_{1}^{-1} L_{\max }^{\frac{1}{2}}\right\rangle+\sum_{2^{30} N_{1} / N_{3} \leq A \leq N_{1}} N_{1}^{-1} N_{3}^{\frac{1}{2}} L_{\max }^{\frac{1}{2}}\right) \prod_{i=1}^{3}\left\|g_{i}\right\|_{L_{\tau}^{2} L_{(d \ell)_{\lambda}}^{2}} \\
& \lesssim L_{\min }^{\frac{1}{2}}\left(\left\langle N_{1}^{-\frac{1}{2}} N_{3}^{-\frac{1}{2}} L_{\max }^{\frac{1}{2}}\right\rangle+\left(\log N_{1}\right) N_{1}^{-1} N_{3}^{\frac{1}{2}} L_{\max }^{\frac{1}{2}}\right) \prod_{i=1}^{3}\left\|g_{i}\right\|_{L_{\tau}^{2} L_{(d \ell)_{\lambda}}^{2}} .
\end{aligned}
$$

For the second term, we find $\left|\Phi\left(\xi_{1}, \eta_{1}, \xi_{2}, \eta_{2}\right)\right| \gtrsim N_{1}^{2} N_{3}$ as in the proof of Proposition 5.17. Then, by using Lemma 5.10 with $P \sim \lambda^{2} N_{3}$, we can verify (58).

Let us turn to Case 2: $\left(\ell_{1}, \ell_{2}\right) \in\left(\mathfrak{D}_{0}^{2^{11}} \times \mathfrak{D}_{0}^{2^{11}}\right) \cup\left(\mathfrak{D}_{2^{10}}^{2^{11}} \times \mathfrak{D}_{2^{10}}^{2^{11}}\right)$. We only consider the case $\left(\ell_{1}, \ell_{2}\right) \in \mathfrak{D}_{0}^{2^{11}} \times \mathfrak{D}_{0}^{2^{11}}$ to reduce redundancy.

Proposition 5.18. Let $\max \left(2^{100}, N_{1} / N_{3}\right) \leq A \leq N_{1}$. Assume that $1 \ll N_{3} \lesssim$ $N_{2} \leq N_{1}, L_{\text {med }} \leq N_{1}^{2},\left(j_{1}, j_{2}\right) \in J_{A}^{0}$ such that $16 \leq\left|j_{1}-j_{2}\right| \leq 32$ and (59). Then we have

$$
\begin{aligned}
& \left.\left|\int_{*} g_{1}\right|_{\tilde{\mathfrak{D}}_{j_{1}}^{A}}\left(\tau_{1}, \ell_{1}\right) g_{2}\right|_{\tilde{\mathfrak{D}}_{j_{2}}^{A}}\left(\tau_{2}, \ell_{2}\right) g_{3}\left(\tau_{3}, \ell_{3}\right)\left(d \tilde{\sigma}_{1}\right)_{\lambda}\left(d \tilde{\sigma}_{2}\right)_{\lambda} \mid \\
& \left.\lesssim L_{\min }^{\frac{1}{2}} \widehat{C}\left(A, N_{1}, N_{3}, L_{\max }\right)\left\|\left.g_{1}\right|_{\tilde{\mathfrak{D}}_{j_{1}}^{A}}\right\|_{L_{\tau}^{2} L_{(d \ell)_{\lambda}}^{2}}\left\|\left.g_{2}\right|_{\tilde{\mathfrak{D}}_{j_{2}}^{A}}\right\|\right|_{L_{\tau}^{2} L_{(d \ell)_{\lambda}}^{2}}\left\|g_{3}\right\|_{L_{\tau}^{2} L_{(d \ell)_{\lambda}}^{2}},
\end{aligned}
$$

where

$$
\widehat{C}\left(A, N_{1}, N_{3}, L_{\max }\right)= \begin{cases}N_{1}^{-1} N_{3}^{\frac{1}{2}} L_{\max }^{\frac{1}{2}} & \text { for } N_{3} \geq 2^{30} A^{-1} N_{1}, \\ N_{3}^{\varepsilon}\left\langle N_{1}^{-\frac{1}{2}} L_{\max }^{\frac{1}{2}}\right\rangle & \text { for } N_{3} \leq 2^{30} A^{-1} N_{1} .\end{cases}
$$

Proof. The case $N_{3} \geq 2^{30} A^{-1} N_{1}$ can be handled in the same manner as in the proof of Proposition 5.17. We focus on the case $N_{3} \leq 2^{30} A^{-1} N_{1}$, which means $A \leq$ $2^{30} N_{1} / N_{3}$. Put $A_{0}=2^{30} N_{1} / N_{3}$ and for a dyadic $K$ such that $2^{10} \leq K \leq 2^{-10} A_{0}$, we define

$$
\begin{aligned}
& \mathfrak{J}_{A_{0}}^{K}=\left\{j \in \mathbb{N} \mid \frac{A_{0}}{K} \leq j \leq 2 \frac{A_{0}}{K}, \quad A_{0}-2 \frac{A_{0}}{K} \leq j \leq A_{0}-\frac{A_{0}}{K}\right\}, \\
& \mathfrak{J}_{A_{0}}=\left\{j \in \mathbb{N} \mid 0 \leq j \leq 2^{10}, \quad A_{0}-2^{10} \leq j \leq A_{0}-1\right\} .
\end{aligned}
$$

Since $N_{1} / N_{3} \leq A \leq 2^{30} N_{1} / N_{3}=A_{0}$, it suffices to show that for $j_{1} \in \mathfrak{J}_{A_{0}}^{K} \cup \mathfrak{J}_{A_{0}}$ and $\left|j_{1}-j_{2}\right| \sim 1$, it holds

$$
\begin{aligned}
& \left.\left|\int_{*} g_{1}\right|_{\tilde{\mathfrak{D}}_{j_{1}}^{A_{0}}}\left(\tau_{1}, \ell_{1}\right) g_{2}\right|_{\tilde{\mathfrak{D}}_{j_{2}}^{A_{0}}}\left(\tau_{2}, \ell_{2}\right) g_{3}\left(\tau_{3}, \ell_{3}\right)\left(d \tilde{\sigma}_{1}\right)_{\lambda}\left(d \tilde{\sigma}_{2}\right)_{\lambda} \mid \\
\lesssim & N_{3}^{\varepsilon} L_{\min }^{\frac{1}{2}}\left\langle N_{1}^{-\frac{1}{2}} L_{\text {max }}^{\frac{1}{2}}\right\rangle\left\|\left.g_{1}\right|_{\tilde{\mathfrak{D}}_{j_{1}}^{A_{0}}}\right\|_{L_{\tau}^{2} L_{(d \ell)_{\lambda}}^{2}}\left\|\left.g_{2}\right|_{\tilde{\mathfrak{D}}_{j_{2}}^{A_{0}}}\right\|_{L_{\tau}^{2} L_{(d \ell)_{\lambda}}^{2}}\left\|g_{3}\right\|_{L_{\tau}^{2} L_{(d \ell)_{\lambda}}^{2}} .
\end{aligned}
$$

We divide the proof of (63) into four cases:

(1) $1 \ll N_{3} \lesssim N_{1}^{\frac{1}{2}}, j_{1} \in \mathfrak{J}_{A_{0}}^{K}$ with $1 \ll K \ll N_{3}$,

(2) $1 \ll N_{3} \lesssim N_{1}^{\frac{1}{2}}, j_{1} \in \mathfrak{J}_{A_{0}}^{K} \cup \mathfrak{J}_{A_{0}}$ with $N_{3} \lesssim K \lesssim A_{0}$,

(3) $N_{1}^{\frac{1}{2}} \lesssim N_{3} \ll N_{1}, j_{1} \in \mathfrak{J}_{A_{0}}^{K}$ with $1 \ll K \ll A_{0}$, 
(4) $N_{1}^{\frac{1}{2}} \lesssim N_{3} \ll N_{1}, j_{1} \in \mathfrak{J}_{A_{0}}^{K} \cup \mathfrak{J}_{A_{0}}$ with $K \sim A_{0}$.

Case (1) Note that $j_{1} \in \mathfrak{J}_{A_{0}}^{K}$ with $\left|j_{1}-j_{2}\right| \sim 1$ implies $\left|\eta_{1}\right|+\left|\eta_{2}\right| \lesssim K^{-1} N_{1}$ for

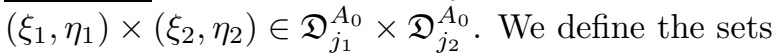

$$
\begin{aligned}
& S_{a}=\left\{\left(\tau, \ell_{(1)}, \ell_{(2)}\right) \in \mathbb{R} \times M\left(\mathbb{Z}^{2} / \lambda\right)|| \ell_{(1)} \mid \leq a^{-1} N_{3}\right\}, \\
& \tilde{S}_{a}=\left\{\left(\tau, \ell_{(1)}, \ell_{(2)}\right) \in \mathbb{R} \times M\left(\mathbb{Z}^{2} / \lambda\right)\left|a^{-1} N_{3} \leq\right| \ell_{(1)} \mid \leq 2 a^{-1} N_{3}\right\} .
\end{aligned}
$$

First we assume supp $g_{3} \subset S_{2^{-30} K}$ and prove (63) by Proposition 5.8 with $A=$ $A_{0} K \sim K N_{1} / N_{3}$. We deduce from $\left|j_{1}-j_{2}\right| \sim 1$ and $\operatorname{supp} g_{3} \subset S_{2^{-10} K}$ that, after harmless decompositions, we can assume that for $i=1,2,3, \operatorname{supp}_{k} g_{i}$ are confined to the rectangle set

$$
K_{i}=\left\{(\xi, \eta) \in \mathbb{R}^{2}|| \xi-\alpha_{i}\left|\ll K^{-1} N_{3} \sim\left(A_{0} K\right)^{-1} N_{1},\right| \eta-\beta_{i} \mid \ll A_{0}^{-1} N_{1}\right\},
$$

with some fixed $\left(\alpha_{i}, \beta_{i}\right) \in \mathbb{R}^{2}$ such that $\left|\beta_{i}\right| \lesssim K^{-1} N_{1}$, respectively.

Since $\partial_{\eta} \tilde{\psi}(\xi, \eta)=3 \eta^{2}$, this implies (60) with $A=A_{0} K$. Next we show the transversality condition (61). It is clear that $\left(\xi_{1}, \eta_{1}\right) \times\left(\xi_{2}, \eta_{2}\right) \in \mathfrak{D}_{j_{1}}^{A_{0}} \times \mathfrak{D}_{j_{2}}^{A_{0}}$ gives $\left|\xi_{1} \eta_{2}-\xi_{2} \eta_{1}\right| \gtrsim A_{0}^{-1} N_{1}^{2}$. Furthermore, we see

$$
\begin{aligned}
\left|\xi_{1} \eta_{2}+\xi_{2} \eta_{1}+2\left(\xi_{1} \eta_{1}+\xi_{2} \eta_{2}\right)\right| & \geq 2\left|\xi_{1} \eta_{1}+\left(\xi_{1}+\xi_{2}\right) \eta_{2}\right|-\left|\xi_{1} \eta_{2}-\xi_{2} \eta_{1}\right| \\
& \geq 2^{-3} K^{-1} N_{1}^{2}-2^{40} K^{-2} N_{1} N_{3}-2^{10} A_{0}^{-1} N_{1}^{2} \\
& \gtrsim K^{-1} N_{1}^{2} .
\end{aligned}
$$

Here we used $K \ll A_{0}$. Hence we can utilize Proposition 5.8 with $A=A_{0} K \sim$ $K N_{1} / N_{3}$ and obtain

$$
\begin{aligned}
& \left.\left|\int_{*} g_{1}\right|_{\tilde{\mathfrak{D}}_{j_{1}}^{A_{0}}}\left(\tau_{1}, \ell_{1}\right) g_{2}\right|_{\tilde{\mathfrak{D}}_{j_{2}}^{A_{0}}\left(\tau_{2}, \ell_{2}\right) g_{3}\left(\tau_{3}, \ell_{3}\right)\left(d \tilde{\sigma}_{1}\right)_{\lambda}\left(d \tilde{\sigma}_{2}\right)_{\lambda} \mid} \mid \\
\lesssim & L_{\min }^{\frac{1}{2}}\left\langle K^{\frac{1}{2}} N_{1}^{-\frac{1}{2}} N_{3}^{-\frac{1}{2}} L_{\text {max }}^{\frac{1}{2}}\right\rangle\left\|g_{1}\right\|_{L_{\tau}^{2} L_{(d \ell)_{\lambda}}^{2}}\left\|g_{2}\right\|_{L_{\tau}^{2} L_{(d \ell)_{\lambda}}^{2}}\left\|g_{3}\right\|_{L_{\tau}^{2} L_{(d \ell)_{\lambda}}^{2}} .
\end{aligned}
$$

Next suppose $\operatorname{supp} g_{3} \subset\left(S_{2^{-30} K}\right)^{c}$. Then we easily observe that $|\Phi| \geq N_{1}^{2}$ which, combined with Lemma 5.10, provides the desired estimate since $N_{3} \lesssim N_{1}^{1 / 2}$.

Case (2) To avoid redundancy, we only treat the case $j_{1} \in \mathfrak{J}_{A_{0}}^{K}$. Assume supp $g_{3} \subset$

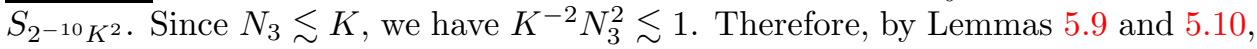
we get

$$
\begin{aligned}
& \left.\left|\int_{*} g_{1}\right|_{\tilde{\mathfrak{D}}_{j_{1}}^{A_{0}}}\left(\tau_{1}, \ell_{1}\right) g_{2}\right|_{\tilde{\mathfrak{D}}_{j_{2}}^{A_{0}}}\left(\tau_{2}, \ell_{2}\right) g_{3}\left(\tau_{3}, \ell_{3}\right)\left(d \tilde{\sigma}_{1}\right)_{\lambda}\left(d \tilde{\sigma}_{2}\right)_{\lambda} \mid \\
\lesssim & L_{\min }^{\frac{1}{2}}\left\|g_{1}\right\|_{L_{\tau}^{2} L_{(d \ell)_{\lambda}}^{2}}\left\|g_{2}\right\|_{L_{\tau}^{2} L_{(d \ell)_{\lambda}}^{2}}\left\|g_{3}\right\|_{L_{\tau}^{2} L_{(d \ell)_{\lambda}}^{2}} .
\end{aligned}
$$

In the case supp $g_{3} \subset \tilde{S}_{\alpha^{-1} K^{2}}$ with $2^{10} \leq \alpha \lesssim K^{2}$, we can observe that $|\Phi| \gtrsim$ $\alpha K^{-2} N_{1}^{2} N_{3}$. In addition, Lemma 5.9 provides $\# \operatorname{supp}_{k} g_{3} \lesssim \lambda^{2} \alpha K^{-2} N_{3}^{2}$. Hence, by employing Lemma 5.10, we have

$$
\begin{aligned}
& \left.\left|\int_{*} g_{1}\right|_{\tilde{\mathfrak{D}}_{j_{1}}^{A_{0}}}\left(\tau_{1}, \ell_{1}\right) g_{2}\right|_{\tilde{\mathfrak{D}}_{j_{2}}^{A_{0}}}\left(\tau_{2}, \ell_{2}\right) g_{3}\left(\tau_{3}, \ell_{3}\right)\left(d \sigma_{1}\right)_{\lambda}\left(d \sigma_{2}\right)_{\lambda} \mid \\
\lesssim & N_{3}^{\frac{1}{2}} N_{1}^{-1} L_{\min }^{\frac{1}{2}} L_{\max }^{\frac{1}{2}}\left\|g_{1}\right\|_{L_{\tau}^{2} L_{(d \ell)_{\lambda}}^{2}}\left\|g_{2}\right\|_{L_{\tau}^{2} L_{(d \ell)_{\lambda}}^{2}}\left\|g_{3}\right\|_{L_{\tau}^{2} L_{(d \ell)_{\lambda}}^{2}} .
\end{aligned}
$$


Consequently, for supp $g_{3} \subset\left(S_{2^{-10} K^{2}}\right)^{c}$, by summing up the above, we get

$$
\begin{aligned}
& \left.\left|\int_{*} g_{1}\right|_{\tilde{\mathfrak{D}}_{j_{1}}^{A_{0}}}\left(\tau_{1}, \ell_{1}\right) g_{2}\right|_{\tilde{\mathfrak{D}}_{j_{2}}^{A_{0}}}\left(\tau_{2}, \ell_{2}\right) g_{3}\left(\tau_{3}, \ell_{3}\right)\left(d \tilde{\sigma}_{1}\right)_{\lambda}\left(d \tilde{\sigma}_{2}\right)_{\lambda} \mid \\
\lesssim & N_{3}^{\frac{1}{2}} N_{1}^{-1+\varepsilon} L_{\min }^{\frac{1}{2}} L_{\max }^{\frac{1}{2}}\left\|g_{1}\right\|_{L_{\tau}^{2} L_{(d \ell)_{\lambda}}^{2}}\left\|g_{2}\right\|_{L_{\tau}^{2} L_{(d \ell)_{\lambda}}^{2}}\left\|g_{3}\right\|_{L_{\tau}^{2} L_{(d \ell)_{\lambda}}^{2}} .
\end{aligned}
$$

Case (3) The case supp $g_{3} \subset S_{2^{-30} \mathrm{~K}}$ can be handled in the same way as in Case (1) and the case $\operatorname{supp} g_{3} \subset\left(S_{2^{-30} K}\right)^{c}$ is treated as in the proof of Case (2). We omit the details.

Case (4) Since $K \sim A_{0}$, we treat only $j_{1} \in \mathfrak{J}_{A_{0}}$ here. We will see that Case (4) is the most difficult part in the proof of Proposition 5.18 and we need to perform an additional Whitney-type decomposition as in [17].

First, we assume supp $g_{3} \subset S_{2^{-10} N_{1}^{2} / N_{3}^{2}}$. We introduce the Whitney-type decomposition of $\mathbb{R}^{2} \times \mathbb{R}^{2}$ into rectangle tiles.

Definition 5.19. Let $1 \lesssim d \lesssim N_{3}^{2} / N_{1}$ be dyadic and $m=\left(m_{(1)}, m_{(2)}\right) \in \mathbb{Z}^{2}$. We define rectangle-tiles $\left\{\mathcal{R}_{m}^{d}\right\}_{m \in \mathbb{Z}^{2}}$ whose short side is parallel to $\xi$-axis and its length is $d^{-1} N_{1}^{-2} N_{3}^{3}$, long side length is $d^{-1} N_{3}$ and prisms $\left\{\tilde{\mathcal{R}}_{m}^{d}\right\}_{m \in \mathbb{Z}^{2}}$ as follows:

$$
\begin{aligned}
& \mathcal{R}_{m}^{d}:=\left\{(\xi, \eta) \in \mathbb{R}^{2} \mid \xi \in d^{-1} N_{1}^{-2} N_{3}^{3}\left[m_{(1)}, m_{(1)}+1\right), \eta \in d^{-1} N_{3}\left[m_{(2)}, m_{(2)}+1\right)\right\}, \\
& \tilde{\mathcal{R}}_{m}^{d}:=\mathbb{R} \times \mathcal{R}_{m}^{d} .
\end{aligned}
$$

Definition 5.20 (Whitney type decomposition). Let $1 \lesssim d \lesssim N_{3}^{2} / N_{1}$ be dyadic and $j_{1} \in \mathfrak{J}_{A_{0}}$. Recall that

$$
\begin{aligned}
& \Phi\left(\xi_{1}, \eta_{1}, \xi_{2}, \eta_{2}\right)=\xi_{1} \xi_{2}\left(\xi_{1}+\xi_{2}\right)+\eta_{1} \eta_{2}\left(\eta_{1}+\eta_{2}\right), \\
& F\left(\xi_{1}, \eta_{1}, \xi_{2}, \eta_{2}\right)=\xi_{1} \eta_{2}+\xi_{2} \eta_{1}+2\left(\xi_{1} \eta_{1}+\xi_{2} \eta_{2}\right) .
\end{aligned}
$$

We define $Z_{d, j_{1}, j_{2}}^{1}$ as the set of $\left(m_{1}, m_{2}\right) \in \mathbb{Z}^{2} \times \mathbb{Z}^{2}$ such that

$$
\left\{\begin{array}{l}
\left|\Phi\left(\xi_{1}, \eta_{1}, \xi_{2}, \eta_{2}\right)\right| \geq d^{-1} N_{3}^{3} \text { for any }\left(\xi_{1}, \eta_{1}\right) \times\left(\xi_{2}, \eta_{2}\right) \in \mathcal{R}_{m_{1}}^{d} \times \mathcal{R}_{m_{2}}^{d} \\
\left(\mathcal{R}_{m_{1}}^{d} \times \mathcal{R}_{m_{2}}^{d}\right) \cap\left(\mathfrak{D}_{j_{1}}^{A_{0}} \times \mathfrak{D}_{j_{2}}^{A_{0}}\right) \neq \emptyset \\
\left|\xi_{1}+\xi_{2}\right| \lesssim N_{1}^{-2} N_{3}^{3} \text { for any }\left(\xi_{1}, \eta_{1}\right) \times\left(\xi_{2}, \eta_{2}\right) \in \mathcal{R}_{m_{1}}^{d} \times \mathcal{R}_{m_{2}}^{d} .
\end{array}\right.
$$

Similarly, we define $Z_{d, j_{1}, j_{2}}^{2}$ as the set of $\left(k_{1}, k_{2}\right) \in \mathbb{Z}^{2} \times \mathbb{Z}^{2}$ such that

$$
\left\{\begin{array}{l}
\left|F\left(\xi_{1}, \eta_{1}, \xi_{2}, \eta_{2}\right)\right| \geq d^{-1} N_{1} N_{3} \text { for any }\left(\xi_{1}, \eta_{1}\right) \times(\xi, \eta) \in \mathcal{R}_{m_{1}}^{d} \times \mathcal{R}_{m_{2}}^{d}, \\
\left(\mathcal{R}_{m_{1}}^{d} \times \mathcal{R}_{m_{2}}^{d}\right) \cap\left(\mathfrak{D}_{j_{1}}^{A_{0}} \times \mathfrak{D}_{j_{2}}^{A_{0}}\right) \neq \emptyset \\
\left|\xi_{1}+\xi_{2}\right| \lesssim N_{1}^{-2} N_{3}^{3} \text { for any }\left(\xi_{1}, \eta_{1}\right) \times\left(\xi_{2}, \eta_{2}\right) \in \mathcal{R}_{m_{1}}^{d} \times \mathcal{R}_{m_{2}}^{d},
\end{array}\right.
$$

and

$$
Z_{d}^{j_{1}, j_{2}}=Z_{d, j_{1}, j_{2}}^{1} \cup Z_{d, j_{1}, j_{2}}^{2}, \quad R_{d}^{j_{1}, j_{2}}=\bigcup_{\left(m_{1}, m_{2}\right) \in Z_{d}^{j_{1}, j_{2}}} \mathcal{R}_{m_{1}}^{d} \times \mathcal{R}_{m_{2}}^{d} \subset \mathbb{R}^{2} \times \mathbb{R}^{2} .
$$

It is clear that $d_{1} \leq d_{2} \Longrightarrow R_{d_{1}}^{j_{1}, j_{2}} \subset R_{d_{2}}^{j_{1}, j_{2}}$. Further, we define

$$
Q_{d}^{j_{1}, j_{2}}= \begin{cases}R_{d}^{j_{1}, j_{2}} \backslash R_{d / 2}^{j_{1}, j_{2}} & \text { for } d \geq 2^{21}, \\ R_{2^{20}}^{j_{1}, j_{2}} & \text { for } d=2^{20},\end{cases}
$$


and a set of pairs of integer coordinates $\widehat{Z}_{d}^{j_{1}, j_{2}} \subset Z_{d}^{j_{1}, j_{2}}$ as

$$
\bigcup_{\left(m_{1}, m_{2}\right) \in \widehat{Z}_{d}^{j_{1}, j_{2}}} \mathcal{R}_{m_{1}}^{d} \times \mathcal{R}_{m_{2}}^{d}=Q_{d}^{j_{1}, j_{2}}
$$

Clearly, $\widehat{Z}_{d}^{j_{1}, j_{2}}$ is uniquely defined and

$$
d_{1} \neq d_{2} \Longrightarrow Q_{d_{1}}^{j_{1}, j_{2}} \cap Q_{d_{2}}^{j_{1}, j_{2}}=\emptyset, \quad \bigcup_{2^{20} \leq d \leq d_{0}} Q_{d}^{j_{1}, j_{2}}=R_{d_{0}}^{j_{1}, j_{2}}
$$

where $d_{0} \gtrsim 1$ is dyadic. Lastly, we define $\bar{Z}_{d}^{j_{1}, j_{2}}$ as the collection of $\left(m_{1}, m_{2}\right) \in$ $\mathbb{Z}^{2} \times \mathbb{Z}^{2}$ which satisfies

$$
\left\{\begin{array}{l}
\mathcal{R}_{m_{1}}^{d} \times \mathcal{R}_{m_{2}}^{d} \not \subset \bigcup_{2^{20} \leq d^{\prime} \leq d} \bigcup_{\left(m_{1}^{\prime}, m_{2}^{\prime}\right) \in \widehat{Z}_{A, d^{\prime}}^{j_{1}, j_{2}}}\left(\mathcal{R}_{m_{1}^{\prime}}^{A, d^{\prime}} \times \mathcal{R}_{m_{2}^{\prime}}^{A, d^{\prime}}\right), \\
\left(\mathcal{R}_{m_{1}}^{d} \times \mathcal{R}_{m_{2}}^{d}\right) \cap\left(\mathfrak{D}_{j_{1}}^{A} \times \mathfrak{D}_{j_{2}}^{A}\right) \neq \emptyset \\
\left|\xi_{1}+\xi_{2}\right| \lesssim N_{1}^{-2} N_{3}^{3} \text { for any }\left(\xi_{1}, \eta_{1}\right) \times\left(\xi_{2}, \eta_{2}\right) \in \mathcal{R}_{m_{1}}^{d} \times \mathcal{R}_{m_{2}}^{d} .
\end{array}\right.
$$

The following lemma ensures the almost orthogonality of rectangle sets $\mathcal{R}_{m_{1}}^{d}$ and $\mathcal{R}_{m_{2}}^{d}$ such that $\left(m_{1}, m_{2}\right) \in \widehat{Z}_{d}^{j_{1}, j_{2}}$ or $\left(m_{1}, m_{2}\right) \in \bar{Z}_{d}^{j_{1}, j_{2}}$. We note that the proof is almost the same as that for Lemmas 3.7 and 3.24 in [17].

Lemma 5.21. Let $1 \lesssim d \lesssim N_{3}^{2} / N_{1}$ be dyadic and $j_{1} \in \mathfrak{J}_{A_{0}}$. For fixed $m_{1} \in \mathbb{Z}^{2}$, the number of $m_{2} \in \mathbb{Z}^{2}$ such that $\left(m_{1}, m_{2}\right) \in \widehat{Z}_{d}^{j_{1}, j_{2}}$ is less than $2^{1000}$. Furthermore, the same claim holds true if we replace $\widehat{Z}_{d}^{j_{1}, j_{2}}$ by $\bar{Z}_{d}^{j_{1}, j_{2}}$.

Proof. Clearly, we can assume $d \geq 2^{100}$. For $\left(m_{1}, m_{2}\right) \in \widehat{Z}_{d}^{j_{1}, j_{2}}$, we can find $m_{1}^{\prime}=$ $m_{1}^{\prime}\left(m_{1}\right) \in \mathbb{Z}^{2}$ and $m_{2}^{\prime}=m_{2}^{\prime}\left(m_{2}\right) \in \mathbb{Z}^{2}$ which satisfy $\mathcal{R}_{m_{1}}^{d} \subset \mathcal{R}_{m_{1}^{\prime}}^{d / 2}$ and $\mathcal{R}_{m_{2}}^{d} \subset \mathcal{R}_{m_{2}^{\prime}}^{d / 2}$, respectively. In view of the definitions, $\left(m_{1}, m_{2}\right) \in \widehat{Z}_{d}^{j_{1}, j_{2}}$ implies that there exist $\left(\bar{\xi}_{1}, \bar{\eta}_{1}\right),\left(\tilde{\xi}_{1}, \tilde{\eta}_{1}\right) \in \mathcal{R}_{m_{1}^{\prime}}^{d / 2},\left(\bar{\xi}_{2}, \bar{\eta}_{2}\right),\left(\tilde{\xi}_{2}, \tilde{\eta}_{2}\right) \in \mathcal{R}_{m_{2}^{\prime}}^{d / 2}$ which satisfy

$$
\left|\Phi\left(\bar{\xi}_{1}, \bar{\eta}_{1}, \bar{\xi}_{2}, \bar{\eta}_{2}\right)\right| \leq 2 d^{-1} N_{3}^{3} \quad \text { and } \quad\left|F\left(\tilde{\xi}_{1}, \tilde{\eta}_{1}, \tilde{\xi}_{2}, \tilde{\eta}_{2}\right)\right| \leq 2 d^{-1} N_{1} N_{3}
$$

Define $\left(\xi_{1}^{\prime}, \eta_{1}^{\prime}\right)$ as the center of $\mathcal{R}_{m_{1}}^{d}$. Since $j_{1} \in \mathfrak{J}_{A_{0}},\left|j_{1}-j_{2}\right| \sim 1$ and $\left(\mathcal{R}_{m_{1}}^{d} \times \mathcal{R}_{m_{2}}^{d}\right) \cap$ $\left(\mathfrak{D}_{j_{1}}^{A} \times \mathfrak{D}_{j_{2}}^{A}\right) \neq \emptyset$, we have $\left|\eta_{1}\right|+\left|\eta_{2}\right| \leq N_{3}$ for any $\left(\xi_{1}, \eta_{1}\right) \times\left(\xi_{2}, \eta_{2}\right) \in \mathcal{R}_{m_{1}}^{d} \times \mathcal{R}_{m_{2}}^{d}$. Therefore, for $\left(\xi_{2}, \eta_{2}\right) \in \mathcal{R}_{m_{2}}^{d}$, (64) implies

$$
\left|\Phi\left(\xi_{1}^{\prime}, \eta_{1}^{\prime}, \xi_{2}, \eta_{2}\right)\right| \leq 2^{5} d^{-1} N_{3}^{3} \quad \text { and } \quad\left|F\left(\xi_{1}^{\prime}, \eta_{1}^{\prime}, \xi_{2}, \eta_{2}\right)\right| \leq 2^{5} d^{-1} N_{1} N_{3}
$$

Consequently, it suffices to see that there exist $\tilde{m}_{1}, \tilde{m}_{2} \in \mathbb{Z}^{2}$ such that $\mathcal{R}_{\tilde{m}_{1}}^{2^{-20} d} \cup$ $\mathcal{R}_{\tilde{m}_{2}}^{2-20} d$ contains

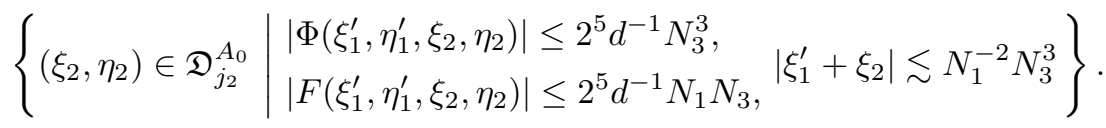

Now let us perform the transformation $\xi_{2}^{\prime}=\xi_{2}+\xi_{1}^{\prime} / 2, \eta_{2}^{\prime}=\eta_{2}+\eta_{1}^{\prime} / 2$ and see that

$$
\begin{aligned}
& \left|\Phi\left(\xi_{1}^{\prime}, \eta_{1}^{\prime}, \xi_{2}, \eta_{2}\right)\right|=\left|\xi_{1}^{\prime} \xi_{2}\left(\xi_{1}^{\prime}+\xi_{2}\right)+\eta_{1}^{\prime} \eta_{2}\left(\eta_{1}^{\prime}+\eta_{2}\right)\right| \leq 2^{5} d^{-1} N_{3}^{3} \\
& \left|F\left(\xi_{1}^{\prime}, \eta_{1}^{\prime}, \xi_{2}, \eta_{2}\right)\right|=\left|\xi_{1}^{\prime} \eta_{2}+\xi_{2} \eta_{1}^{\prime}+2\left(\xi_{1}^{\prime} \eta_{1}^{\prime}+\xi_{2} \eta_{2}\right)\right| \leq 2^{5} d^{-1} N_{1} N_{3}
\end{aligned}
$$


are rewritten as

$$
\begin{aligned}
& \tilde{\Phi}\left(\xi_{2}^{\prime}, \eta_{2}^{\prime}\right):=\left|\xi_{1}^{\prime} \xi_{2}^{\prime 2}+\eta_{1}^{\prime} \eta_{2}^{2}-\frac{\xi_{1}^{3}+\eta_{1}^{\prime 3}}{4}\right| \leq 2^{5} d^{-1} N_{3}^{3}, \\
& \tilde{F}\left(\xi_{2}^{\prime}, \eta_{2}^{\prime}\right):=\left|\frac{3}{2} \xi_{1}^{\prime} \eta_{1}^{\prime}+2 \xi_{2}^{\prime} \eta_{2}^{\prime}\right| \leq 2^{5} d^{-1} N_{1} N_{3},
\end{aligned}
$$

respectively. It should be noted that $\left|\xi_{1}^{\prime}+\xi_{2}\right| \lesssim N_{1}^{-2} N_{3}^{3}$ provides $\left|\xi_{2}^{\prime}\right| \geq\left|\xi_{2}\right| / 2-$ $\left|\xi_{1}^{\prime}+\xi_{2}\right| / 2 \geq 2^{-2} N_{1}$. We compute that

$$
(66) \Longrightarrow\left|\eta_{2}^{\prime}+\frac{3 \xi_{1}^{\prime} \eta_{1}^{\prime}}{4 \xi_{2}^{\prime}}\right| \leq \frac{2^{4} d^{-1} N_{1} N_{3}}{\left|\xi_{2}^{\prime}\right|} \leq 2^{6} d^{-1} N_{3} .
$$

Since $\left|\eta_{1}^{\prime}\right| \leq N_{3}$, (65) and (67) yield

$$
\begin{gathered}
\left|\xi_{1}^{\prime}{\xi_{2}^{\prime}}^{2}+\eta_{1}^{\prime}{\eta_{2}^{\prime}}^{2}-\frac{\xi_{1}^{\prime 3}+\eta_{1}^{3}}{4}\right| \leq 2^{5} d^{-1} N_{3}^{3} \\
\stackrel{(67)}{\Longrightarrow}\left|\xi_{1}^{\prime}{\xi_{2}^{\prime}}^{2}+\eta_{1}^{\prime} \frac{9 \xi_{1}^{\prime 2}{\eta_{1}^{\prime}}^{2}}{16{\xi_{2}^{\prime}}^{2}}-\frac{\xi_{1}^{3}+\eta_{1}^{\prime 3}}{4}\right| \leq 2^{8} d^{-1} N_{3}^{3} .
\end{gathered}
$$

We define

$$
G\left(\xi_{2}^{\prime}\right):=\xi_{1}^{\prime}{\xi_{2}^{\prime}}^{2}+\frac{9 \xi_{1}^{\prime 2}{\eta_{1}^{\prime}}^{3}}{16{\xi_{2}^{\prime}}^{2}}-\frac{\xi_{1}^{3}+\eta_{1}^{\prime 3}}{4} .
$$

It follows from $2^{-2} N_{1} \leq\left|\xi_{1}^{\prime}\right|,\left|\xi_{2}^{\prime}\right| \leq 2 N_{1}$ and $\left|\eta_{1}^{\prime}\right| \leq N_{3}$ that

$$
\left|\left(\frac{d G}{d \xi_{2}^{\prime}}\right)\left(\xi_{2}^{\prime}\right)\right|=\left|\frac{2 \xi_{1}^{\prime}}{\xi_{2}^{\prime 3}}\left(\xi_{2}^{\prime 4}-\frac{9 \xi_{1}^{\prime} \eta_{1}^{\prime 3}}{16}\right)\right| \geq 2^{-5} N_{1}^{2} .
$$

This and (68) verify that there exist constants $c_{1}\left(\xi_{1}^{\prime}, \eta_{1}^{\prime}\right), c_{2}\left(\xi_{1}^{\prime}, \eta_{1}^{\prime}\right) \in \mathbb{R}$ such that

$$
\min _{i=1,2}\left|\xi_{2}^{\prime}-c_{i}\left(\xi_{1}^{\prime}, \eta_{1}^{\prime}\right)\right| \leq 2^{15} d^{-1} N_{1}^{-2} N_{3}^{3} .
$$

This and (67) imply that there exist constants $c_{1}^{\prime}\left(\xi_{1}^{\prime}, \eta_{1}^{\prime}\right), c_{2}^{\prime}\left(\xi_{1}^{\prime}, \eta_{1}^{\prime}\right) \in \mathbb{R}$ such that

$$
\min _{i=1,2}\left|\eta_{2}^{\prime}-c_{i}^{\prime}\left(\xi_{1}^{\prime}, \eta_{1}^{\prime}\right)\right| \leq 2^{7} d^{-1} N_{3},
$$

which completes the proof.

Lemma 5.22. Let $1 \lesssim d \lesssim N_{3}^{2} / N_{1}$ be dyadic, $j_{1} \in \mathfrak{J}_{A_{0}}$. Assume that $\left|j_{1}-j_{2}\right| \sim 1$ and $\left(m_{1}, m_{2}\right) \in \widehat{Z}_{d}^{j_{1}, j_{2}}$. Then we have

$$
\begin{aligned}
& \left.\left|\int_{*} g_{1}\right|_{\tilde{\mathcal{R}}_{m_{1}}^{d}}\left(\tau_{1}, \ell_{1}\right) g_{2}\right|_{\tilde{\mathcal{R}}_{m_{2}}^{d}}\left(\tau_{2}, \ell_{2}\right) g_{3}\left(\tau_{3}, \ell_{3}\right)\left(d \tilde{\sigma}_{1}\right)_{\lambda}\left(d \tilde{\sigma}_{2}\right)_{\lambda} \mid \\
& \lesssim L_{\min }^{\frac{1}{2}}\left\langle\left(d^{\frac{1}{2}} N_{3}^{-1}+d^{-\frac{1}{2}} N_{1}^{-1} N_{3}^{\frac{1}{2}}\right) L_{\max }^{\frac{1}{2}}\right\rangle\left\|g_{1}\right\|_{L_{\tau}^{2} L_{(d \ell)_{\lambda}}^{2}}\left\|g_{2}\right\|_{L_{\tau}^{2} L_{(d \ell)_{\lambda}}^{2}}\left\|g_{3}\right\|_{L_{\tau}^{2} L_{(d \ell)_{\lambda}}^{2}} .
\end{aligned}
$$

Proof. First we assume $\left|\Phi\left(\xi_{1}, \eta_{1}, \xi_{2}, \eta_{2}\right)\right| \geq d^{-1} N_{3}^{3}$ for any $\left(\xi_{1}, \eta_{1}\right) \times\left(\xi_{2}, \eta_{2}\right) \in \mathcal{R}_{m_{1}}^{d} \times$ $\mathcal{R}_{m_{2}}^{d}$. By Lemma 5.9, we have $\sup _{\alpha \in \mathbb{R}^{2}} \# \tilde{R}_{d^{-1} N_{3}, d^{-1} N_{1}^{-2} N_{3}^{3}} \lesssim \lambda^{2} d^{-2} N_{1}^{-2} N_{3}^{4}$. Therefore, it follows from Lemma 5.10 that

$$
\begin{aligned}
& \left.\left|\int_{*} g_{1}\right|_{\tilde{\mathcal{R}}_{m_{1}}^{d}}\left(\tau_{1}, \ell_{1}\right) g_{2}\right|_{\tilde{\mathcal{R}}_{m_{2}}^{d}}\left(\tau_{2}, \ell_{2}\right) g_{3}\left(\tau_{3}, \ell_{3}\right)\left(d \tilde{\sigma}_{1}\right)_{\lambda}\left(d \tilde{\sigma}_{2}\right)_{\lambda} \mid \\
& \lesssim d^{-\frac{1}{2}} N_{1}^{-1} N_{3}^{\frac{1}{2}} L_{\min }^{\frac{1}{2}} L_{\max }^{\frac{1}{2}} \prod_{i=1}^{3}\left\|g_{i}\right\|_{L_{\tau}^{2} L_{(d \ell)_{\lambda}}^{2}} .
\end{aligned}
$$


For the case $\left|F\left(\xi_{1}, \eta_{1}, \xi_{2}, \eta_{2}\right)\right| \geq d^{-1} N_{1} N_{3}$, it follows from Proposition 5.8 with $A=d A_{0} N_{1} / N_{3} \sim d N_{1}^{2} / N_{3}^{2}$ that

$$
\begin{aligned}
& \left.\left|\int_{*} g_{1}\right|_{\tilde{\mathcal{R}}_{m_{1}}^{d}}\left(\tau_{1}, \ell_{1}\right) g_{2}\right|_{\tilde{\mathcal{R}}_{m_{2}}^{d}}\left(\tau_{2}, \ell_{2}\right) g_{3}\left(\tau_{3}, \ell_{3}\right)\left(d \tilde{\sigma}_{1}\right)_{\lambda}\left(d \tilde{\sigma}_{2}\right)_{\lambda} \mid \\
\lesssim & L_{\min }^{\frac{1}{2}}\left\langle d^{\frac{1}{2}} N_{3}^{-1} L_{\text {max }}^{\frac{1}{2}}\right\rangle \prod_{i=1}^{3}\left\|g_{i}\right\|_{L_{\tau}^{2} L_{(d \ell)_{\lambda}}^{2}} .
\end{aligned}
$$

This completes the proof.

We turn to show (63) for Case (4) under the assumption supp $g_{3} \subset S_{2^{-10} N_{1}^{2} / N_{3}^{2}}$. By the properties of $\widehat{Z}_{d}^{j_{1}, j_{2}}$ and $\bar{Z}_{d}^{j_{1}, j_{2}}$, we observe that

$$
\begin{aligned}
& (\text { LHS ) of (63) } \\
\leq & \left.\sum_{1 \lesssim d \lesssim N_{3}^{2} / N_{1}} \sum_{\left(m_{1}, m_{2}\right) \in \widehat{Z}_{d}^{j_{1}, j_{2}}}\left|\int_{*} g_{1}\right|_{\tilde{\mathcal{R}}_{m_{1}}^{d}}\left(\tau_{1}, \ell_{1}\right) g_{2}\right|_{\tilde{\mathcal{R}}_{m_{2}}^{d}}\left(\tau_{2}, \ell_{2}\right) g_{3}\left(\tau_{3}, \ell_{3}\right)\left(d \tilde{\sigma}_{1}\right)_{\lambda}\left(d \tilde{\sigma}_{2}\right)_{\lambda} \mid \\
+ & \left.\sum_{\left(m_{1}, m_{2}\right) \in \bar{Z}_{N_{3}^{2} / N_{1}}^{j_{1}, j_{2}}}\left|\int_{*} g_{1}\right|_{\tilde{\mathcal{R}}_{m_{1}}^{N_{3}^{2} / N_{1}}}\left(\tau_{1}, \ell_{1}\right) g_{2}\right|_{\tilde{\mathcal{R}}_{m_{2}}^{N_{3}^{2} / N_{1}}}\left(\tau_{2}, \ell_{2}\right) g_{3}\left(\tau_{3}, \ell_{3}\right)\left(d \tilde{\sigma}_{1}\right)_{\lambda}\left(d \tilde{\sigma}_{2}\right)_{\lambda} \mid \\
= & \sum_{1 \lesssim d \lesssim N_{3}^{2} / N_{1}} \sum_{\left(m_{1}, m_{2}\right) \in \widehat{Z}_{d}^{j_{1}, j_{2}}} I_{1}+\sum_{\left(m_{1}, m_{2}\right) \in \bar{Z}_{N_{3}^{2} / N_{1}}^{j_{1}, j_{2}}} I_{2} .
\end{aligned}
$$

The first is estimated by Lemmas 5.21, 5.22 as

$$
\sum_{1 \lesssim d \lesssim N_{3}^{2} / N_{1}} \sum_{\left(m_{1}, m_{2}\right) \in \widehat{Z}_{d}^{j_{1}, j_{2}}} I_{1} \lesssim\left(\log N_{3}\right) L_{\min }^{\frac{1}{2}}\left\langle N_{1}^{-\frac{1}{2}} L_{\max }^{\frac{1}{2}}\right\rangle \prod_{i=1}^{3}\left\|g_{i}\right\|_{L_{\tau}^{2} L_{(d \ell)_{\lambda}}^{2}} .
$$

For the second term, since Lemma 5.9 provides $\sup _{\alpha \in \mathbb{R}^{2}} \# \tilde{R}_{N_{1} N_{3}^{-1}, N_{1}^{-1} N_{3}}^{\alpha} \lesssim 1$, Lemmas 5.10 and 5.21 establish

$$
\sum_{\left(m_{1}, m_{2}\right) \in \bar{Z}_{N_{3}^{2} / N_{1}}^{j_{1}, j_{2}}} I_{2} \lesssim L_{\min }^{\frac{1}{2}}\left\|g_{1}\right\|_{L_{\tau}^{2} L_{(d \ell)_{\lambda}}^{2}}\left\|g_{2}\right\|_{L_{\tau}^{2} L_{(d \ell)_{\lambda}}^{2}}\left\|g_{3}\right\|_{L_{\tau}^{2} L_{(d \ell)_{\lambda}}^{2}} .
$$

Lastly, we treat the case $\operatorname{supp} g_{3} \subset\left(S_{2^{-10} N_{1}^{2} / N_{3}^{2}}\right)^{c}$. Let us assume $\operatorname{supp} g_{3} \subset$ $\tilde{S}_{\alpha^{-1} N_{1}^{2} / N_{3}^{2}}$ with $2^{10} \leq \alpha \lesssim N_{1}^{2} / N_{3}^{2}$.

This condition gives $|\Phi| \gtrsim \alpha N_{3}^{3}$ and $\# \operatorname{supp}_{k} g_{3} \lesssim \lambda^{2} \alpha N_{3}^{4} / N_{1}^{2}$. Thus, by Lemma 5.9 , we obtain

$$
\begin{aligned}
& \left.\left|\int_{*} g_{1}\right|_{\tilde{\mathfrak{D}}_{j_{1}}^{A_{0}}}\left(\tau_{1}, \ell_{1}\right) g_{2}\right|_{\tilde{\mathfrak{D}}_{j_{2}}^{A_{0}}}\left(\tau_{2}, \ell_{2}\right) g_{3}\left(\tau_{3}, \ell_{3}\right)\left(d \tilde{\sigma}_{1}\right)_{\lambda}\left(d \tilde{\sigma}_{2}\right)_{\lambda} \mid \\
& \lesssim N_{3}^{\frac{1}{2}} N_{1}^{-1} L_{\min }^{\frac{1}{2}} L_{\max }^{\frac{1}{2}} \prod_{i=1}^{3}\left\|g_{i}\right\|_{L_{\tau}^{2} L_{(d \ell)_{\lambda}}^{2}} .
\end{aligned}
$$

Consequently, if supp $g_{3} \subset\left(S_{2^{-10} N_{1}^{2} / N_{3}^{2}}\right)^{c}$ by summing up the above, we get

$$
\begin{aligned}
& \left.\left|\int_{*} g_{1}\right|_{\tilde{\mathfrak{D}}_{j_{1}}^{A_{0}}}\left(\tau_{1}, \ell_{1}\right) g_{2}\right|_{\tilde{\mathfrak{D}}_{j_{2}}^{A_{0}}}\left(\tau_{2}, \ell_{2}\right) g_{3}\left(\tau_{3}, \ell_{3}\right)\left(d \tilde{\sigma}_{1}\right)_{\lambda}\left(d \tilde{\sigma}_{2}\right)_{\lambda} \mid \\
\lesssim & N_{3}^{\frac{1}{2}} N_{1}^{-1+\varepsilon} L_{\min }^{\frac{1}{2}} L_{\max }^{\frac{1}{2}} \prod_{i=1}^{3}\left\|g_{i}\right\|_{L_{\tau}^{2} L_{(d \ell)_{\lambda}}^{2}} .
\end{aligned}
$$


Proof of (58) for the case $\left(\ell_{1}, \ell_{2}\right) \in \mathfrak{D}_{0}^{2^{11}} \times \mathfrak{D}_{0}^{2^{11}}$. We can see

$$
\begin{aligned}
& \left.\left|\int_{*} g_{1}\right|_{\tilde{\mathfrak{D}}_{0}^{211}}\left(\tau_{1}, \ell_{1}\right) g_{2}\right|_{\tilde{\mathfrak{D}}_{0}^{21}}\left(\tau_{2}, \ell_{2}\right) g_{3}\left(\tau_{3}, \ell_{3}\right)\left(d \tilde{\sigma}_{1}\right)_{\lambda}\left(d \tilde{\sigma}_{2}\right)_{\lambda} \mid \\
\lesssim & \left(\sum_{N_{1} / N_{3} \leq A \leq 2^{30} N_{1} / N_{3}}+\sum_{2^{30} N_{1} / N_{3} \leq A \leq N_{1}}\right) \sum_{\substack{\left(j_{1}, j_{2}\right) \in J_{A}^{0} \\
16 \leq\left|j_{1}-j_{2}\right| \leq 32}} I_{A}^{j_{1}, j_{2}}+\sum_{\substack{\left(j_{1}, j_{2}\right) \in J_{N_{1}}^{0} \\
\left|j_{1}-j_{2}\right| \leq 16}} I_{N_{1}}^{j_{1}, j_{2}} .
\end{aligned}
$$

It follows from Proposition 5.18 that

$$
\begin{aligned}
& \left(\sum_{N_{1} / N_{3} \leq A \leq 2^{30} N_{1} / N_{3}}+\sum_{2^{30} N_{1} / N_{3} \leq A \leq N_{1}}\right) \sum_{\substack{\left(j_{1}, j_{2}\right) \in J_{0}^{j} \\
16 \leq\left|j_{1}-j_{2}\right| \leq 32}} I_{A}^{j_{1}, j_{2}} \\
& \lesssim L_{\min }^{\frac{1}{2}}\left(\sum_{A \sim N_{1} / N_{3}} N_{3}^{\varepsilon}\left\langle N_{1}^{-\frac{1}{2}} L_{\max }^{\frac{1}{2}}\right\rangle+\sum_{2^{30} N_{1} / N_{3} \leq A \leq N_{1}} N_{1}^{-1} N_{3}^{\frac{1}{2}} L_{\max }^{\frac{1}{2}}\right) \prod_{i=1}^{3}\left\|g_{i}\right\|_{L_{\tau}^{2} L_{(d \ell)_{\lambda}}^{2}} \\
& \lesssim L_{\min }^{\frac{1}{2}}\left(N_{3}^{\varepsilon}\left\langle N_{1}^{-\frac{1}{2}} L_{\text {max }}^{\frac{1}{2}}\right\rangle+\left(\log N_{1}\right) N_{1}^{-1} N_{3}^{\frac{1}{2}} L_{\max }^{\frac{1}{2}}\right) \prod_{i=1}^{3}\left\|g_{i}\right\|_{L_{\tau}^{2} L_{(d \ell)_{\lambda}}^{2}} .
\end{aligned}
$$

The second term is handled in the same manner as in the proof for the case $\left(\ell_{1}, \ell_{2}\right) \in \mathfrak{D}_{j}^{2^{11}} \times \mathfrak{D}_{j}^{2^{11}}$ with fixed $j \neq 0,2^{9} \times 3,2^{10}$.

It remains to prove Proposition 5.2 under the assumption $\max \left(\left|k_{1,1}\right|,\left|k_{2,1}\right|\right) \leq$ $2^{-5} N_{1}$.

Proof of Proposition 5.2 for the case (II). First we treat non-parallel interactions. Let $N_{1} / N_{3} \leq A \leq N_{1}$ and $16 \leq\left|j_{1}-j_{2}\right| \leq 32$. We prove

$$
\begin{aligned}
& \left.\left|\int_{*}\left(\left|k_{3,1}\right|+\left|k_{1,1}\right| \frac{N_{3}}{N_{1}}\right) f_{1}\right|_{\tilde{\mathfrak{D}}_{j_{1}}^{A}}\left(\tau_{1}, k_{1}\right) f_{2}\right|_{\tilde{\mathfrak{D}}_{j_{2}}^{A}}\left(\tau_{2}, k_{2}\right) f_{3}\left(\tau_{3}, k_{3}\right)\left(d \sigma_{1}\right)_{\lambda}\left(d \sigma_{2}\right)_{\lambda} \mid \\
& \lesssim A^{-\frac{1}{2}}\left(N_{1} N_{3}\right)^{\frac{1}{2}} L_{\min }^{\frac{1}{2}}\left\langle N_{1}^{-\frac{1}{2}} L_{\max }^{\frac{1}{2}}\right\rangle \prod_{i=1}^{3}\left\|f_{i}\right\|_{L_{\tau}^{2} L_{(d k)_{\lambda}}^{2}} .
\end{aligned}
$$

By symmetry, we can always assume $\left|k_{1,1}\right| \geq\left|k_{2,1}\right|$ and then there exists a dyadic number $2^{5} \leq \alpha \leq A$ such that $\left|k_{1,1}\right| \sim \alpha^{-1} N_{1}$. We divide the proof of (69) into the two cases $\left|k_{3,2}\right| \lesssim \alpha A^{-1} N_{1}$ and $\left|k_{3,2}\right| \gg \alpha A^{-1} N_{1}$.

In the first case, we shall see that the condition $\left|k_{3,2}\right| \lesssim \alpha A^{-1} N_{1}$ gives $\left|k_{3,1}\right| \lesssim$ $A^{-1} N_{1}$. Let $\left(r_{k} \cos \theta_{k}, r_{k} \sin \theta_{k}\right) \in \mathfrak{D}_{j_{k}}^{A}$, where $k=1,2$, satisfy $\left(r_{1} \cos \theta_{1}, r_{1} \sin \theta_{1}\right)+$ $\left(r_{2} \cos \theta_{2}, r_{2} \sin \theta_{2}\right) \in \operatorname{supp}_{k} f_{3}$. Clearly, $\left|\cos \theta_{1}+\cos \theta_{2}\right| \lesssim A^{-1}$ and $\left|\cos \theta_{1}\right| \lesssim \alpha^{-1}$. Further, since $\left|k_{3,2}\right| \lesssim \alpha A^{-1} N_{1}$, it holds $\left|r_{1}-r_{2}\right| \lesssim \alpha A^{-1} N_{1}$. Therefore, we get

$$
\begin{aligned}
\left|r_{1} \cos \theta_{1}+r_{2} \cos \theta_{2}\right| & \leq\left|\left(r_{1}-r_{2}\right) \cos \theta_{1}\right|+r_{2}\left|\cos \theta_{1}+\cos \theta_{2}\right| \\
& \lesssim A^{-1} N_{1} .
\end{aligned}
$$

Hence, (69) is proved by

$$
\begin{aligned}
& \left.\left|\int_{*} f_{1}\right|_{\tilde{\mathfrak{D}}_{j_{1}}^{A}}\left(\tau_{1}, k_{1}\right) f_{2}\right|_{\tilde{\mathfrak{D}}_{j_{2}}^{A}}\left(\tau_{2}, k_{2}\right) f_{3}\left(\tau_{3}, k_{3}\right)\left(d \sigma_{1}\right)_{\lambda}\left(d \sigma_{2}\right)_{\lambda} \mid \\
& \lesssim A^{\frac{1}{2}} N_{1}^{-\frac{1}{2}} N_{3}^{\frac{1}{2}} L_{\min }^{\frac{1}{2}}\left\langle N_{1}^{-\frac{1}{2}} L_{\max }^{\frac{1}{2}}\right\rangle \prod_{i=1}^{3}\left\|f_{i}\right\|_{L_{\tau}^{2} L_{(d k)_{\lambda}}^{2}} .
\end{aligned}
$$


To see this, we decompose $k_{3,2}$ by employing

$$
\mathbb{S}_{A}^{m}=\left\{\eta \in \mathbb{R}\left|m A^{-1} N_{1} \leq\right| \eta \mid \leq(m+1) A^{-1} N_{1}\right\},
$$

where $m \in \mathbb{N}_{0}$. Since $\left|k_{3,2}\right| \lesssim N_{3}$, we have $\left\{k_{3,2}\right\} \subset \bigcup_{m \lesssim A N_{3} / N_{1}} \mathbb{S}_{A}^{m}$. Then, for fixed $m$, it suffices to show

$$
\begin{aligned}
& \left.\left|\int_{*} \chi_{\mathbb{S}_{A}^{m}}\left(k_{3,2}\right) f_{1}\right|_{\tilde{\mathfrak{D}}_{j_{1}}^{A}}\left(\tau_{1}, k_{1}\right) f_{2}\right|_{\tilde{\mathfrak{D}}_{j_{2}}^{A}}\left(\tau_{2}, k_{2}\right) f_{3}\left(\tau_{3}, k_{3}\right)\left(d \sigma_{1}\right)_{\lambda}\left(d \sigma_{2}\right)_{\lambda} \mid \\
& \lesssim L_{\min }^{\frac{1}{2}}\left\langle A^{\frac{1}{2}} N_{1}^{-1} L_{\max }^{\frac{1}{2}}\right\rangle \prod_{i=1}^{3}\left\|f_{i}\right\|_{L_{\tau}^{2} L_{(d k)_{\lambda}}^{2}} .
\end{aligned}
$$

This can be obtained by Proposition 5.3. We omit the details.

Next we assume $\left|k_{3,2}\right| \gg \alpha A^{-1} N_{1}$. Since $\left|k_{3,2}\right| \lesssim N_{3}$ we can assume $A \gg$ $\alpha N_{1} / N_{3}$. The above observation (70) implies $\left|k_{3,1}\right| \lesssim \alpha^{-1} N_{3}$ and $\left|k_{3,2}\right| \sim N_{3}$. Let

$$
\widehat{\Phi}=\widehat{\Phi}\left(\xi_{1}, \eta_{1}, \xi_{2}, \eta_{2}\right)=3 \xi_{1} \xi_{2}\left(\xi_{1}+\xi_{2}\right)+\xi_{1} \eta_{2}\left(2 \eta_{1}+\eta_{2}\right)+\xi_{2} \eta_{1}\left(\eta_{1}+2 \eta_{2}\right) .
$$

For all $\left(\xi_{k}, \eta_{k}\right) \in \mathfrak{D}_{j_{k}}^{A}$ such that $\left(\xi_{1}+\xi_{2}, \eta_{1}+\eta_{2}\right) \in \operatorname{supp}_{k} f_{3}$, we will show $|\widehat{\Phi}| \gtrsim$ $\alpha^{-1} N_{1}^{2} N_{3}$ which implies $L_{\max } \gtrsim \alpha^{-1} N_{1}^{2} N_{3}$. To show this, we first observe that

$$
\begin{aligned}
& \left|\xi_{1} \eta_{2}\left(2 \eta_{1}+\eta_{2}\right)+\xi_{2} \eta_{1}\left(\eta_{1}+2 \eta_{2}\right)\right| \\
= & \left|\frac{3}{2}\left(\xi_{1} \eta_{2}+\xi_{2} \eta_{1}\right)\left(\eta_{1}+\eta_{2}\right)+\frac{\xi_{1} \eta_{2}-\xi_{2} \eta_{1}}{2}\left(\eta_{1}-\eta_{2}\right)\right| \\
\geq & \left|\left(\xi_{1} \eta_{2}+\xi_{2} \eta_{1}\right)\left(\eta_{1}+\eta_{2}\right)\right|-\left|\left(\eta_{1}-\eta_{2}\right)\left(\xi_{1} \eta_{2}-\xi_{2} \eta_{1}\right)\right| \\
\gtrsim & \alpha^{-1} N_{1}^{2} N_{3} .
\end{aligned}
$$

Here we used $A^{-1} N_{1} \ll\left|\eta_{1}+\eta_{2}\right| \sim N_{3}$ and $\left|\xi_{1} \eta_{2}-\xi_{2} \eta_{1}\right| \lesssim A^{-1} N_{1}^{2}$. We calculate

$$
\begin{aligned}
|\widehat{\Phi}| & =\left|3 \xi_{1} \xi_{2}\left(\xi_{1}+\xi_{2}\right)+\xi_{1} \eta_{2}\left(2 \eta_{1}+\eta_{2}\right)+\xi_{2} \eta_{1}\left(\eta_{1}+2 \eta_{2}\right)\right| \\
& \geq\left|\xi_{1} \eta_{2}\left(2 \eta_{1}+\eta_{2}\right)+\xi_{2} \eta_{1}\left(\eta_{1}+2 \eta_{2}\right)\right|-3\left|\xi_{1} \xi_{2}\left(\xi_{1}+\xi_{2}\right)\right| \\
& \gtrsim \alpha^{-1} N_{1}^{2} N_{3} .
\end{aligned}
$$

Note that \# $\operatorname{supp}_{k} f_{3} \lesssim \lambda^{2} A^{-1} N_{1} N_{3}$. Consequently, by Lemma 5.7 , we see that $\left|k_{3,1}\right| \lesssim \alpha^{-1} N_{3}, L_{\max } \gtrsim \alpha^{-1} N_{1}^{2} N_{3}$ and $\# \operatorname{supp}_{k} f_{3} \lesssim \lambda^{2} A^{-1} N_{1} N_{3}$ yield (69).

Next we treat parallel interactions. We show the following equation with $\mid j_{1}-$ $j_{2} \mid \leq 16$.

$$
\begin{aligned}
& \left.\left|\int_{*}\left(\left|k_{3,1}\right|+\left|k_{1,1}\right| \frac{N_{3}}{N_{1}}\right) f_{1}\right|_{\tilde{\mathfrak{D}}_{j_{1}}^{N_{1}}}\left(\tau_{1}, k_{1}\right) f_{2}\right|_{\tilde{\mathfrak{D}}_{j_{2}}^{N_{1}}}\left(\tau_{2}, k_{2}\right) f_{3}\left(\tau_{3}, k_{3}\right)\left(d \sigma_{1}\right)_{\lambda}\left(d \sigma_{2}\right)_{\lambda} \mid \\
\lesssim & N_{3}^{\frac{1}{2}} L_{\min }^{\frac{1}{2}}\left\langle N_{1}^{-\frac{1}{2}} L_{\max }^{\frac{1}{2}}\right\rangle \prod_{i=1}^{3}\left\|f_{i}\right\|_{L_{\tau}^{2} L_{(d k)_{\lambda}}^{2}} .
\end{aligned}
$$

The proof is almost the same as that for (69). If $\left|k_{1,1}\right| \lesssim 1$, we easily confirm (72) since $\left|k_{3,1}\right| \sim 1$ and $\# \operatorname{supp}_{k} f_{3} \lesssim \lambda^{2} N_{3}$. Let $2^{5} \leq \alpha \leq N_{1}$ and suppose $\left|k_{1,1}\right| \sim \alpha^{-1} N_{1}$. As for the non-parallel case, the proof is divided into the cases $\left|k_{3,2}\right| \lesssim \alpha$ and $\left|k_{3,2}\right| \gg \alpha$. The first is dealt with the observation (70), which provides $\left|k_{3,1}\right| \lesssim 1$, and $\# \operatorname{supp}_{k} f_{3} \lesssim \lambda^{2} N_{3}$. The second can be handled by the same argument as for the proof of (69) in the case $\left|k_{3,2}\right| \gg \alpha A^{-1} N_{1}$. 
Now we complete the proof of Proposition 5.2 by using (69) and (72). The assumption $\max \left(\left|k_{1,1}\right|,\left|k_{2,1}\right|\right) \leq 2^{-5} N_{1}$ suggests $\left(k_{1}, k_{2}\right) \in \mathfrak{D}_{2^{4}}^{2^{5}} \times \mathfrak{D}_{2^{4}}^{2^{5}}$. Let us recall the Whitney type decomposition of angular variables. Define

$$
J_{A}=\left\{\left(j_{1}, j_{2}\right) \mid 0 \leq j_{1}, j_{2} \leq A-1, \quad\left(\mathfrak{D}_{j_{1}}^{A} \times \mathfrak{D}_{j_{2}}^{A}\right) \subset\left(\mathfrak{D}_{2^{4}}^{2^{5}} \times \mathfrak{D}_{2^{4}}^{2^{5}}\right) .\right\}
$$

It is observed that

$$
\mathfrak{D}_{2^{4}}^{2^{5}} \times \mathfrak{D}_{2^{4}}^{2^{5}}=\bigcup_{2^{8} \leq A \leq N_{1}} \bigcup_{\begin{array}{c}
\left(j_{1}, j_{2}\right) \in J_{A} \\
16 \leq\left|j_{1}-j_{2}\right| \leq 32
\end{array}} \mathfrak{D}_{j_{1}}^{A} \times \mathfrak{D}_{j_{2}}^{A} \cup \bigcup_{\begin{array}{c}
\left(j_{1}, j_{2}\right) \in J_{N} \\
\left|j_{1}-j_{2}\right| \leq 16
\end{array}} \mathfrak{D}_{j_{1}}^{N_{1}} \times \mathfrak{D}_{j_{2}}^{N_{1}} .
$$

Thus, if we write

$$
\tilde{I}_{A}^{j_{1}, j_{2}}:=\left.\left|\int_{*}\left(\left|k_{3,1}\right|+\left|k_{1,1}\right| \frac{N_{3}}{N_{1}}\right) f_{1}\right|_{\tilde{\mathfrak{D}}_{j_{1}}^{A}}\left(\tau_{1}, k_{1}\right) f_{2}\right|_{\tilde{\mathfrak{D}}_{j_{2}}^{A}}\left(\tau_{2}, k_{2}\right) f_{3}\left(\tau_{3}, k_{3}\right)\left(d \sigma_{1}\right)_{\lambda}\left(d \sigma_{2}\right)_{\lambda} \mid,
$$

we have

$$
\begin{aligned}
& \left.\left|\int_{*}\left(\left|k_{3,1}\right|+\left|k_{1,1}\right| \frac{N_{3}}{N_{1}}\right) f_{1}\right|_{\tilde{\mathfrak{D}}_{2^{4}}^{25}}\left(\tau_{1}, k_{1}\right) f_{2}\right|_{\tilde{\mathfrak{D}}_{2^{4}}^{25}}\left(\tau_{2}, k_{2}\right) f_{3}\left(\tau_{3}, k_{3}\right)\left(d \sigma_{1}\right)_{\lambda}\left(d \sigma_{2}\right)_{\lambda} \mid \\
\lesssim & \sum_{N_{1} / N_{3} \leq A \leq N_{1}} \sum_{\substack{\left(j_{1}, j_{2}\right) \in J_{A} \\
1 \leq \leq\left|j_{1}-j_{2}\right| \leq 32}} I_{A}^{j_{1}, j_{2}}+\sum_{\substack{\left(j_{1}, j_{2}\right) \in J_{N_{1}} \\
\left|j_{1}-j_{2}\right| \leq 16}} I_{N_{1}}^{j_{1}, j_{2}} .
\end{aligned}
$$

The first term is handled by (69) and the second term is estimated by (72), respectively.

Remark 5.23. (i). We end this section with an example indicating sharpness of Proposition 5.2 up to endpoints. Firstly, consider the symmetrized equation

$$
\partial_{t} u+\left(\partial_{x}^{3}+\partial_{y}^{3}\right) u=u\left(\partial_{x}+\partial_{y}\right) u, \quad(t, x, y) \in \mathbb{R} \times \mathbb{T}^{2} .
$$

In Subsection 4.4 we have seen that the frequencies $(N,-N),(N, 2 N),(2 N, N)$ yield a fully transverse interaction, i.e., $A \sim 1$ in (49), with vanishing resonance $\Phi=0$. We find with $f_{i}$ supported on the above modes

$$
\begin{aligned}
&\left|\int_{\mathbb{R} \times \mathbb{Z}^{2}} f_{1}\left(\tau_{1}, k_{1}\right) f_{2}\left(\tau_{2}, k_{2}\right)\left(k_{3,1}+k_{3,2}\right) f_{3}\left(\tau_{3}, k_{3}\right)\left(d \sigma_{1}\right)_{1}\left(d \sigma_{2}\right)_{1}\right| \\
& \sim N L_{\min }^{1 / 2} \prod_{i=1}^{3}\left\|f_{i}\right\|_{L_{\tau}^{2} L_{(d k)}^{2}} .
\end{aligned}
$$

(ii). For $L_{\text {med }}=L_{\max }=T(N)^{-1}$, which is the minimal modulation for the corresponding time localization due to (7),

$$
\begin{aligned}
& \left|\int_{\mathbb{R} \times \mathbb{Z}^{2}} f_{1}\left(\tau_{1}, k_{1}\right) f_{2}\left(\tau_{2}, k_{2}\right)\left(k_{3,1}+k_{3,2}\right) f_{3}\left(\tau_{3}, k_{3}\right)\left(d \sigma_{1}\right)_{1}\left(d \sigma_{2}\right)_{1}\right| \\
& \lesssim N\left(L_{1} L_{2} L_{3}\right)^{1 / 2} T(N) \prod_{i=1}^{3}\left\|f_{i}\right\|_{L_{\tau}^{2} L_{(d k)_{1}}^{2}} .
\end{aligned}
$$

This extends to the unsymmetrized equation by rational approximation. Let $\varepsilon>0$. Consider $p_{n}, q_{n} \in \mathbb{N}$ with

$$
\left|\sqrt{3}-\frac{p_{n}}{q_{n}}\right| \leq \varepsilon
$$


As frequency modes for the unsymmetrized Zakharov-Kuznetsov equation choose $\left(0,2 p_{n}\right),\left(3 q_{n},-p_{n}\right),\left(3 q_{n}, p_{n}\right)$. Clearly, $\Phi=0$. We find for the frequencies after symmetrization

$$
\begin{aligned}
& k_{1}=\left(\frac{2 p_{n}}{\sqrt{3}}, \frac{-2 p_{n}}{\sqrt{3}}\right), k_{2}=\left(3 q_{n}-\frac{p_{n}}{\sqrt{3}}, 3 q_{n}+\frac{p_{n}}{\sqrt{3}}\right), \\
& k_{3}=\left(3 q_{n}+\frac{p_{n}}{\sqrt{3}}, 3 q_{n}-\frac{p_{n}}{\sqrt{3}}\right) .
\end{aligned}
$$

And we compute with $F$ from Section 5 , quantifying transversality, $F=18 q_{n}^{2}-p_{n}^{2}=$ $O\left(N^{2}\right)$ full transversality of the frequency modes. Taking $p_{n}, q_{n} \rightarrow \infty$ yields the claim.

\section{Norm INFLATION FOR COMPLEX-VALUED INITIAL DATA}

In the following we give two examples of complex-valued initital data exhibiting norm inflation. We have already mentioned that this in sharp contrast to the $\mathbb{R}^{2}$ case as also for complex-valued initial data local well-posedness was proved in [17] for $s>-1 / 4$. The below considerations are inspired by [10], where related examples were considered for a quadratic Schrödinger equation with derivative nonlinearity.

The following initial data will give rise to norm inflation in any Sobolev space. However, it seems to be highly pathological:

$$
u_{0}(t, x, y)=i A+B e^{i N x} .
$$

From the form of the interaction it is easy to infer that the excited modes are precisely of the form $(N k, 0)$ for $k \in \mathbb{N}_{0}$. And moreover, the Fourier coefficient for $(N, 0)$ satisfies the differential equation (due to vanishing resonance):

$$
\partial_{t} \hat{u}(t, N, 0)=N A \hat{u}(t, N, 0),
$$

which yields the exponential growth $\hat{u}(t, N, 0)=e^{t N A} i B$. Setting $A=\varepsilon$ and $B=\varepsilon N^{-s}$, we easily see that $\left\|u_{0}\right\|_{H^{s}} \lesssim \varepsilon$, however already for time-scales $\varepsilon$ the $H^{s}$-norm is bounded from below by $\gtrsim \varepsilon^{-1}$.

One can avoid the zero Fourier coefficient for another family of frequency modes with vanishing resonance, namely $(0,2),(N,-1),(N, 1)$ (cf. [22]) to find norm inflation for the initial data

$$
u_{0}(x, y)=A e^{2 i y}+B e^{i(N x-y)} .
$$

By the above means, we infer that

$$
\hat{u}(t, N,-1)=(-i N) t A B,
$$

and $\|u\|_{H^{s}} \gtrsim \varepsilon^{2} N|t|$ again giving norm inflation, e.g., for $t=\varepsilon, N=\varepsilon^{-4}$.

\section{ACKNOWLEDGEMENTS}

The first author acknowledges support by the German Research Foundation (DFG) through the CRC 1283, and the second author is supported through postdoctoral start-up funding by the DFG following his graduation within the IRTG 2235. We would like to thank Professor Sebastian Herr for helpful discussions on Loomis-Whitney-type inequalities and Dr. Tristan Robert for useful remarks on diophantine approximation. 


\section{REFERENCES}

[1] I. Bejenaru, S. Herr, J. Holmer, and D. Tataru. On the 2D Zakharov system with $L^{2}$ Schrödinger data. Nonlinearity, 22(5):1063-1089, 2009.

[2] Ioan Bejenaru and Sebastian Herr. Convolutions of singular measures and applications to the Zakharov system. J. Funct. Anal., 261(2):478-506, 2011.

[3] Ioan Bejenaru, Sebastian Herr, and Daniel Tataru. A convolution estimate for two-dimensional hypersurfaces. Rev. Mat. Iberoam., 26(2):707-728, 2010.

[4] Matania Ben-Artzi, Herbert Koch, and Jean-Claude Saut. Dispersion estimates for third order equations in two dimensions. Comm. Partial Differential Equations, 28(11-12):19431974, 2003.

[5] Jonathan Bennett and Neal Bez. Some nonlinear Brascamp-Lieb inequalities and applications to harmonic analysis. J. Funct. Anal., 259(10):2520-2556, 2010.

[6] Jonathan Bennett, Anthony Carbery, and Terence Tao. On the multilinear restriction and Kakeya conjectures. Acta Math., 196(2):261-302, 2006.

[7] Jonathan Bennett, Anthony Carbery, and James Wright. A non-linear generalisation of the Loomis-Whitney inequality and applications. Math. Res. Lett., 12(4):443-457, 2005.

[8] J. L. Bona and R. Smith. The initial-value problem for the Korteweg-de Vries equation. Philos. Trans. Roy. Soc. London Ser. A, 278(1287):555-601, 1975.

[9] Anthony Carbery. A multilinear generalisation of the Cauchy-Schwarz inequality. Proc. Amer. Math. Soc., 132(11):3141-3152, 2004.

[10] Michael Christ. Ill-posedness of a Schrödinger equation with derivative nonlinearity. 2003. available at http://math.berkeley.edu/ mchrist/Papers/dnls.ps.

[11] J. Colliander, M. Keel, G. Staffilani, H. Takaoka, and T. Tao. Sharp global well-posedness for KdV and modified KdV on $\mathbb{R}$ and T. J. Amer. Math. Soc., 16(3):705-749, 2003.

[12] A. V. Faminskiǔ. The Cauchy problem for the Zakharov-Kuznetsov equation. Differentsial'nye Uravneniya, 31(6):1070-1081, 1103, 1995.

[13] Axel Grünrock and Sebastian Herr. The Fourier restriction norm method for the ZakharovKuznetsov equation. Discrete Contin. Dyn. Syst., 34(5):2061-2068, 2014.

[14] Zihua Guo and Tadahiro Oh. Non-existence of solutions for the periodic cubic NLS below $L^{2}$. Int. Math. Res. Not. IMRN, (6):1656-1729, 2018.

[15] A. D. Ionescu, C. E. Kenig, and D. Tataru. Global well-posedness of the KP-I initial-value problem in the energy space. Invent. Math., 173(2):265-304, 2008.

[16] Carlos E. Kenig, Gustavo Ponce, and Luis Vega. Well-posedness and scattering results for the generalized Korteweg-de Vries equation via the contraction principle. Comm. Pure Appl. Math., 46(4):527-620, 1993.

[17] Shinya Kinoshita. Global Well-posedness for the Cauchy problem of the Zakharov-Kuznetsov equation in 2D. arXiv e-prints, page arXiv:1905.01490, May 2019.

[18] Herbert Koch and Stefan Steinerberger. Convolution estimates for singular measures and some global nonlinear Brascamp-Lieb inequalities. Proc. Roy. Soc. Edinburgh Sect. A, 145(6):12231237, 2015.

[19] E. Kuznetsov and V. Zakharov. On three dimensional solitons. J. Exp. Theor. Phys., 39:285$286,1974$.

[20] E. W. Laedke and K. H. Spatschek. Nonlinear ion-acoustic waves in weak magnetic fields. Phys. Fluids, 25(6):985-989, 1982.

[21] David Lannes, Felipe Linares, and Jean-Claude Saut. The Cauchy problem for the EulerPoisson system and derivation of the Zakharov-Kuznetsov equation. In Studies in phase space analysis with applications to PDEs, volume 84 of Progr. Nonlinear Differential Equations Appl., pages 181-213. Birkhäuser/Springer, New York, 2013.

[22] Felipe Linares, Mahendra Panthee, Tristan Robert, and Nikolay Tzvetkov. On the periodic Zakharov-Kuznetsov equation. Discrete Contin. Dyn. Syst., 39(6):3521-3533, 2019.

[23] Felipe Linares and Ademir Pastor. Local and global well-posedness for the 2D generalized Zakharov-Kuznetsov equation. J. Funct. Anal., 260(4):1060-1085, 2011.

[24] L. H. Loomis and H. Whitney. An inequality related to the isoperimetric inequality. Bull. Amer. Math. Soc, 55:961-962, 1949.

[25] Luc Molinet. Sharp ill-posedness results for the KdV and $\mathrm{mKdV}$ equations on the torus. Adv. Math., 230(4-6):1895-1930, 2012.

[26] Luc Molinet and Didier Pilod. Bilinear Strichartz estimates for the Zakharov-Kuznetsov equation and applications. Ann. Inst. H. Poincaré Anal. Non Linéaire, 32(2):347-371, 2015. 
[27] Javier Ramos. A trilinear restriction estimate with sharp dependence on transversality. Amer. J. Math., 140(6):1443-1469, 2018.

[28] Robert Schippa. On the Cauchy problem for higher dimensional Benjamin-Ono and ZakharovKuznetsov equations. arXiv e-prints, page arXiv:1903.02027, Mar 2019.

[29] Robert Schippa. Short-time Fourier transform restriction phenomena and applications to nonlinear dispersive equations. PhD thesis, Universität Bielefeld, 2019.

Fakultät für Mathematik, Universität Bielefeld, Postfach 1001 31, 33501 Bielefeld, Germany

E-mail address: kinoshita@math.uni-bielefeld.de

E-mail address: robert.schippa@uni-bielefeld.de 